\title{
TWO-SIDED BGG RESOLUTIONS OF ADMISSIBLE REPRESENTATIONS
}

\author{
TOMOYUKI ARAKAWA
}

\begin{abstract}
We prove the conjecture of Frenkel, Kac and Wakimoto on the existence of two-sided BGG resolutions of $G$-integrable admissible representations of affine Kac-Moody algebras at fractional levels. As an application we establish the semi-infinite analogue of the generalized Borel-Weil theorem for minimal parabolic subalgebras which enables an inductive study of admissible representations.
\end{abstract}

\section{INTRODUCTION}

Wakimoto modules are representations of non-twisted affine Kac-Moody algebras introduced by Wakimoto Wak in the case of $\widehat{\mathfrak{s l}}_{2}$ and by Feigin and Frenkel [FF1] in the general case. Wakimoto modules have useful applications in representation theory and conformal field theory. In these applications it is important to have a resolution of an irreducible highest weight representation $L(\lambda)$ of an affine KacMoody algebra $\mathfrak{g}$ in terms of Wakimoto modules, that is, a complex

$$
C^{\bullet}(\lambda): \rightarrow C^{i-1}(\lambda) \stackrel{d_{i-1}}{\rightarrow} C^{i}(\lambda) \stackrel{d_{i}}{\rightarrow} C^{i+1}(\lambda) \rightarrow \ldots
$$

with a differential $d_{i}$ which is a $\mathfrak{g}$-module homomorphism such that $C^{i}(\lambda)$ is a direct sum of Wakimoto modules and

$$
H^{i}\left(C^{\bullet}(\lambda)\right)= \begin{cases}L(\lambda) & \text { if } i=0, \\ 0 & \text { otherwise }\end{cases}
$$

The existence of such a resolution has been proved by Feigin and Frenkel FF2 for any integrable representations over arbitrary $\mathfrak{g}$ and by Bernard and Felder [BF] and Feigin and Frenkel [FF2] for any admissible representation [KW2] over $\widehat{\mathfrak{s l}}_{2}$. In their study of $W$-algebras Frenkel, Kac and Wakimoto [FKW, Conjecture 3.5.1] conjectured the existence of such a resolution for any principle admissible representations over arbitrary $\mathfrak{g}$. In this paper we prove the existence of a two-sided resolution in terms of Wakimoto modules for any $\mathfrak{g}$-integrable admissible representations over arbitrary $\mathfrak{g}$ (Theorem 6.11), where $\mathfrak{\mathfrak { g }}$ is the classical part of $\mathfrak{g}$. For a general principal admissible representation of $\mathfrak{g}$ we obtain the two-sided resolution in terms of twisted Wakimoto modules (Theorem 6.15).

Received by the editors April 9, 2013 and, in revised form, April 10, 2013 and June 6, 2014. 2010 Mathematics Subject Classification. Primary 06B15, 17B67, 81R10.

This work was partially supported by JSPS KAKENHI Grant Number No. 20340007 and No. 23654006. 
Let us sketch the proof of our result briefly. By Fiebig's equivalence Fie the block of the category $\mathcal{O}$ of $\mathfrak{g}$ containing an admissible representation $L(\lambda)$ is equivalent to the block containing an integrable representation 1 . Therefore an admissible representation admits a usual BGG type resolution in terms of Verma modules by the result of GL, RCW]. Hence the idea of Arkhipov Ark1] is applicable in our situation: One can obtain a twisted BGG resolution of $L(\lambda)$ in terms of twisted Verma modules by applying the twisting functor $T_{w}$ Ark1 to the BGG resolution of $L(\lambda)$ as we have the "Borel-Weil-Bott" vanishing property AS.

$$
\mathcal{L}_{i} T_{w} L(\lambda) \cong \begin{cases}L(\lambda) & \text { if } i=\ell(w) \\ 0 & \text { otherwise }\end{cases}
$$

for $w \in \mathcal{W}(\lambda)$, where $\mathcal{W}(\lambda)$ is the integral Weyl group of $\lambda$ and $\ell: \mathcal{W}(\lambda) \rightarrow \mathbb{Z}_{\geq 0}$ is the length function; see Theorem 5.12 It remains to show that one can construct an inductive system of twisted BGG resolutions $\left\{B_{w}^{\bullet}(\lambda)\right\}$ of $L(\lambda)$ such that the complex $\lim _{\vec{w}} B_{w}^{\bullet}(\lambda)$ gives the required two-sided resolution of $L(\lambda)$; see $₫ 6$ for the details.

We note that by applying the (generalized) quantum Drinfeld-Sokolov reduction functor [FKW, KRW] to the (duals of the) two-sided BGG resolutions of admissible representations we obtain resolutions of some of simple modules over $W$-algebras in terms of free field realizations due to the vanishing of the associated BRST cohomology A1, A2, A3, A4, A5. In particular we obtain two-sided resolutions of all the minimal series representations [FKW, A7] of the $W$-algebras associated with principal nilpotent elements in terms of free bosonic realizations.

As an application of the existence of two-sided BGG resolution for admissible representations we prove a semi-infinite analogue of the generalized Borel-Weil theorem [Kos] for minimal parabolic subalgebras (Theorem 7.7). This result is important since it enables an inductive study of admissible representations; see our subsequent paper [A6].

This paper is organized as follows. In $₫ 2$ we collect and prove some basic results about semi-infinite cohomology [Fel] and semi-regular bimodules [Vor1] which are needed for later use. In particular we establish an important property of semiregular bimodules in Proposition [2.1. In $\$ 2$ we collect basic results on the semiinfinite Bruhat ordering (or the generic Bruhat ordering) of an affine Weyl group defined by Lusztig [Lus and study the semi-infinite analogue of parabolic subgroups. Semi-infinite Bruhat ordering is important for us since it (conjecturally) describes the space of homomorphisms between Wakimoto modules; see Proposition 4.10 and Conjecture 4.11 The semi-infinite analogue of the minimal (or maximal) length representatives (Theorem 3.3) is important for describing the semi-infinite restriction functors studied in 97 . In 4 we define Wakimoto modules and twisted Verma modules following Vor2 and study some of their basic properties. In particular we prove the uniqueness of Wakimoto modules which was stated in FF2 without a proof (Theorem 4.7). In $\$ 5$ we generalize the Borel-Weil-Bott vanishing property of the twisting functor established in [AS] to the affine Kac-Moody algebra cases. In $\$ 6$ we state and prove the main results of this paper. In $\$ 7$ we study the semi-infinite restriction functor and establish the semi-infinite analogue of the

\footnotetext{
${ }^{1}$ In the case $L(\lambda)$ is a non-principal $G$-integrable admissible representation this is a block of another Kac-Moody algebra.
} 
generalized Borel-Weil theorem [Kos for minimal parabolic subalgebras. This is a non-trivial fact since admissible representations are not unitarizable unless they are integrable.

\section{Semi-REgular Bimodules AND SEMi-INFinite COHOMOlOGY}

2.1. Some notation. For $\mathbb{Z}$-graded vector spaces $M=\bigoplus_{n \in \mathbb{Z}} M_{n}, N=\bigoplus_{n \in \mathbb{Z}} N_{n}$ with finite-dimensional homogeneous components let

$$
\begin{aligned}
\mathcal{H o m}_{\mathbb{C}}(M, N) & =\bigoplus_{n \in \mathbb{Z}} \mathcal{H o m}_{\mathbb{C}}(M, N)_{n}, \\
\mathcal{H o m}_{\mathbb{C}}(M, N)_{n} & =\left\{f \in \operatorname{Hom}_{\mathbb{C}}(M, N) ; f\left(M_{i}\right) \subset N_{i+n}\right\}, \\
\mathcal{E}_{n} d_{\mathbb{C}}(M) & =\mathcal{H o m}_{\mathbb{C}}(M, M) .
\end{aligned}
$$

We denote by $M^{*}=\bigoplus_{n \in \mathbb{Z}}\left(M^{*}\right)_{n}$ the space $\mathcal{H o m}_{\mathbb{C}}(M, \mathbb{C})$, where $\mathbb{C}$ is considered as a graded vector space concentrated in the degree 0 component. If $M, N$ are module over an algebra $A$ we denote by $\mathcal{H o m}_{A}(M, N)$ the space of all $A$-homomorphisms in $\mathcal{H o m}_{\mathbb{C}}(M, N)$.

2.2. Semi-infinite structure. Let $\mathfrak{g}$ be a complex Lie algebra. A semi-infinite structure Vor1] of $\mathfrak{g}$ is the following data:

(i) a $\mathbb{Z}$-grading $\mathfrak{g}=\bigoplus_{n \in \mathbb{Z}} \mathfrak{g}_{n}$ of $\mathfrak{g}$ with finite-dimensional homogeneous components, $\operatorname{dim}_{\mathbb{C}} \mathfrak{g}_{n}<\infty$ for all $n$

(ii) a semi-infinite 1-cochain $\gamma: \mathfrak{g} \rightarrow \mathbb{C}$.

Here by a semi-infinite 1-cochain we mean the following: Decompose $\mathfrak{g}$ into the direct sum of two subalgebras

$$
\begin{aligned}
& \mathfrak{g}=\mathfrak{g}_{+} \oplus \mathfrak{g}_{-}, \\
& \mathfrak{g}_{+}=\bigoplus_{i \geq 0} \mathfrak{g}_{i}, \quad \mathfrak{g}_{-}=\bigoplus_{i<0} \mathfrak{g}_{i} .
\end{aligned}
$$

A linear map $\gamma: \mathfrak{g} \rightarrow \mathbb{C}$ is called a semi-infinite 1-cochain if $\gamma$ satisfies

$$
\gamma([x, y])=\operatorname{tr}\left((\operatorname{ad} x)_{+-}(\operatorname{ad} y)_{-+}-(\operatorname{ad} y)_{+-}(\operatorname{ad} x)_{-+}\right) \quad \text { for } x, y \in \mathfrak{g},
$$

where $(\operatorname{ad} x)_{ \pm \mp}$ denotes the composition $\mathfrak{g}_{\mp} \stackrel{\text { ad } x}{\rightarrow} \mathfrak{g} \stackrel{\text { projection }}{\rightarrow} \mathfrak{g}_{ \pm}$.

In the rest of this section we assume that $\mathfrak{g}$ is equipped with a semi-infinite structure such that $\gamma\left(\sum_{i \neq 0} \mathfrak{g}\right)=0$.

We denote by $U, U_{-}, U_{+}$, the enveloping algebras of $\mathfrak{g}, \mathfrak{g}_{+}, \mathfrak{g}_{-}$, respectively. These algebras inherit a $\mathbb{Z}$-grading from the corresponding Lie algebras.

Let $\tilde{\mathcal{O}}^{\mathfrak{g}}$ be the category of $\mathbb{Z}$-graded $\mathfrak{g}$-modules $M=\bigoplus_{n \in \mathbb{Z}} M_{n}$ with $\operatorname{dim} M_{n}<\infty$ for all $m$ on which $\bigoplus_{j>0} \mathfrak{g}_{+}$acts locally nilpotently and $\mathfrak{g}_{0}$ acts locally finitely.

2.3. Semi-infinite cohomology. Choose a basis $\left\{x_{i} ; i \in \mathbb{Z}\right\}$ of $\mathfrak{g}$ such that $\left\{x_{i} ; i \geq 0\right\}$ and $\left\{x_{i} ; i<0\right\}$ are bases of $\mathfrak{g}_{+}$and $\mathfrak{g}_{-}$, respectively, and let $\left\{c_{i j}^{k}\right\}$ be the structure constant: $\left[x_{i}, x_{j}\right]=\sum_{k} c_{i j}^{k} x_{k}$.

Denote by $\mathcal{C} l(\mathfrak{g})$ the Clifford algebra associated with $\mathfrak{g} \oplus \mathfrak{g}^{*}$, which has the following generators and relations:

generators: $\psi_{i}, \psi_{i}^{*}$ for $i \in \mathbb{Z}$, relations: $\left\{\psi_{i}, \psi_{j}^{*}\right\}=\delta_{i, j},\left\{\psi_{i}, \psi_{j}\right\}=\left\{\psi_{i}^{*}, \psi_{j}^{*}\right\}=0$. 
Here $\{X, Y\}=X Y+Y X$. The space of the semi-infinite forms $\bigwedge^{\frac{\infty}{2}+\bullet}(\mathfrak{g})$ of $\mathfrak{g}$ is by definition the irreducible representation of $\mathcal{C l}(\mathfrak{g})$ generated by the vector $\mathbf{1}$ satisfying

$$
\psi_{i} \mathbf{1}=0 \quad \text { for } i \geq 0, \quad \psi_{i}^{*} \mathbf{1}=0 \quad \text { for } i>0 .
$$

It is graded by $\operatorname{deg} \mathbf{1}=0, \operatorname{deg} \psi_{i}^{*}=1$ and $\operatorname{deg} \psi_{i}=-1: \bigwedge^{\frac{\infty}{2}+\bullet}(\mathfrak{g})=\bigoplus_{p \in \mathbb{Z}} \bigwedge^{\frac{\infty}{2}+p}(\mathfrak{g})$.

For $A \in \mathcal{E} n d_{\mathbb{C}}\left(\bigwedge^{\frac{\infty}{2}+\bullet}(\mathfrak{g})\right)$ of degree $n$ set

$$
: \psi_{k} A:=\left\{\begin{array}{ll}
\psi_{k} A & \text { if } k<0, \\
(-1)^{n} A \psi_{k} & \text { if } k \geq 0,
\end{array} \quad: \psi_{k}^{*} A:= \begin{cases}\psi_{k}^{*} A & \text { if } k \leq 0 \\
(-1)^{n} A \psi_{k}^{*} & \text { if } k>0 .\end{cases}\right.
$$

The following defines a $\mathfrak{g}$-module structure on $\bigwedge^{\frac{\infty}{2}+\bullet}(\mathfrak{g})$ :

$$
x_{i} \mapsto: \operatorname{ad}\left(x_{i}\right):+\gamma\left(x_{i}\right),
$$

where

$$
: \operatorname{ad} x_{i}:=\sum_{j, k} c_{i j}^{k}: \psi_{k} \psi_{j}^{*}:
$$

For $M \in \tilde{\mathcal{O}}^{\mathfrak{g}}$, define $d \in \operatorname{End}\left(M \otimes \bigwedge^{\frac{\infty}{2}+\bullet}(\mathfrak{g})\right)$ by

$$
d=\sum_{i \in \mathbb{Z}} x_{i} \otimes \psi_{i}^{*}-1 \otimes \frac{1}{2} \sum_{i, j, k \in \mathbb{Z}} c_{i j}^{k}: \psi_{i}^{*}\left(: \psi_{j}^{*} \psi_{k}:\right):+1 \otimes \sum_{i \in \mathbb{Z}} \gamma\left(x_{i}\right) \psi_{i}^{*} .
$$

Then

$$
d^{2}=0, \quad d\left(M \otimes \bigwedge^{\frac{\infty}{2}+p}(\mathfrak{g})\right) \subset M \otimes \bigwedge^{\frac{\infty}{2}+p+1}(\mathfrak{g})
$$

The cohomology of the complex $\left(M \otimes \bigwedge^{\frac{\infty}{2}+\bullet}(\mathfrak{g}), d\right)$ is called the semi-infinite $\mathfrak{g}$ cohomology with coefficients in $M$ and denoted by $H^{\frac{\infty}{2}+\bullet}(\mathfrak{g}, M)$ ([Fel, Vor1] $)$.

2.4. Semi-regular bimodules. We consider the full dual $\operatorname{space}_{\operatorname{Hom}_{\mathbb{C}}}(U, \mathbb{C})$ of $U$ as a $U$-bimodule by $(X f)(u)=f(u X),(f X)(u)=f(X u)$ for $X \in \mathfrak{g}, f \in$ $\operatorname{Hom}_{\mathbb{C}}(M, \mathbb{C}), u \in U$. The graded duals $U_{ \pm}^{*}$ of $U_{ \pm}$are $\mathfrak{g}_{ \pm}$-submodule of $\operatorname{Hom}_{\mathbb{C}}(U, \mathbb{C})$. By abuse of notation we denote by $U^{*}$ the image of the embedding $U_{+}^{*} \otimes_{\mathbb{C}} U_{-}^{*} \hookrightarrow$ $\operatorname{Hom}_{\mathbb{C}}(U, \mathbb{C}), f_{+} \otimes f_{-} \mapsto\left(u_{-} u_{+} \mapsto f_{+}\left(u_{+}\right) f_{-}\left(u_{-}\right)\right), f_{ \pm} \in U_{ \pm}^{*}, u_{ \pm} \in U$. Then $U^{*}$ is a $U$-bisubmodule of $\operatorname{Hom}_{\mathbb{C}}(U, \mathbb{C})$ and coincides with the image of the embedding $U_{-}^{*} \otimes_{\mathbb{C}} U_{+}^{*} \hookrightarrow \operatorname{Hom}_{\mathbb{C}}(U, \mathbb{C}), f_{-} \otimes f_{+} \mapsto\left(u_{+} u_{-} \mapsto f_{+}\left(u_{+}\right) f_{-}\left(u_{-}\right)\right)$.

Following Vor2 define

$$
U S(\mathfrak{g})=H^{\frac{\infty}{2}+0}\left(\mathfrak{g}, U^{*} \otimes_{\mathbb{C}} U\right),
$$

where $\mathfrak{g}$ is given the opposite semi-infinite structure and the semi-infinite $\mathfrak{g}$-cohomology is taken with respect to the diagonal left action on $U^{*} \otimes_{\mathbb{C}} U$. Here by the opposite semi-infinite structure we mean the one obtained by replacing $\mathfrak{g}_{ \pm}$with $\mathfrak{g}_{\mp}$ and $\gamma$ with $-\gamma$. The space $U S(\mathfrak{g})$ inherits the $U$-bimodule structure from $U^{*} \otimes U$ defined by

$$
X(f \otimes u)=-(f X) \otimes u, \quad(f \otimes u) X=f \otimes(u X)
$$

for $X \in \mathfrak{g}, \in U^{*}, u \in U$. The $U$-bimodule $U S(\mathfrak{g})$ is called the semi-regular bimodule of $\mathfrak{g}$. One has

$$
U S(\mathfrak{g}) \cong U_{+}^{*} \otimes_{U_{+}} U
$$


as left $\mathfrak{g}_{+}$-modules and right $\mathfrak{g}$-modules, and the left $\mathfrak{g}$-module structure of $U S(\mathfrak{g})$ is defined through the isomorphism

$$
U_{+} \otimes_{U_{-}} U \cong \mathcal{H o m}_{\mathbb{C}}\left(U_{+}, U\right) \cong \mathcal{H o m}_{U_{-}}\left(U, U_{-} \otimes_{\mathbb{C}} \mathbb{C}_{-\gamma}\right)
$$

( Vor1, Soe2, Vor2]).

Let $M$ be a $\mathfrak{g}$-module and consider the following four left $\mathfrak{g}$-module structures on $U S(\mathfrak{g}) \otimes_{\mathbb{C}} M$ :

$$
\begin{aligned}
& \pi_{1}(X)(s \otimes m)=-(s X) \otimes m+s \otimes X m, \quad \pi_{2}(X)(s \otimes m)=(X s) \otimes m, \\
& \pi_{1}^{\prime}(X)(s \otimes m)=-(s X) \otimes m, \quad \pi_{2}^{\prime}(X)(s \otimes m)=(X s) \otimes m+s \otimes(X m),
\end{aligned}
$$

for $X \in \mathfrak{g}, s \in U S(\mathfrak{g}), m \in M$. Clearly, the two actions $\pi_{1}$ and $\pi_{2}$ (resp. $\pi_{1}^{\prime}$ and $\left.\pi_{2}^{\prime}\right)$ commute.

Proposition 2.1 (cf. [AG, 6.4]). For $M \in \tilde{\mathcal{O}}^{\mathfrak{g}}$ the two $U$-bimodule structures $\left(\pi_{1}, \pi_{2}\right)$ and $\left(\pi_{1}^{\prime}, \pi_{2}^{\prime}\right)$ on $U S(\mathfrak{g}) \otimes_{\mathbb{C}} M$ are equivalent. Namely there exists a linear isomorphism $\Phi: U S(\mathfrak{g}) \otimes_{\mathbb{C}} M \stackrel{\sim}{\rightarrow} U S(\mathfrak{g}) \otimes_{\mathbb{C}} M$ such that $\Phi \circ \pi_{i}^{\prime}(X)=\pi_{i}(X) \circ \Phi$ for $i=1,2, X \in \mathfrak{g}$.

Proof. Define the linear isomorphism

$$
\tilde{\Phi}_{1}: U^{*} \otimes_{\mathbb{C}} U \otimes_{\mathbb{C}} M \stackrel{\sim}{\rightarrow} U^{*} \otimes_{\mathbb{C}} U \otimes_{\mathbb{C}} M
$$

by $\tilde{\Phi}_{1}(f \otimes u \otimes m)=f \otimes(\Delta(u)(1 \otimes m))$ for $f \in U^{*}, u \in U, m \in M$, where $\Delta: U \rightarrow$ $U \otimes_{\mathbb{C}} U$ is the coproduct. We have

$$
\begin{aligned}
& \tilde{\Phi}_{1} \circ \pi_{2, L}(X)=\left(\pi_{2, L}(X)+\pi_{3, L}(X)\right) \circ \tilde{\Phi}_{1}, \\
& \tilde{\Phi}_{1} \circ\left(\pi_{2, R}(X)+\pi_{3, R}(X)\right)=\pi_{2, R}(X) \circ \tilde{\Phi}_{1},
\end{aligned}
$$

where $\pi_{i, L}$ (resp. $\pi_{i, R}$ ) denotes the left action (resp. the right action) of $\mathfrak{g}$ on the $i$-th factor of $U^{*} \otimes U \otimes M$, and $M$ is considered as a right $\mathfrak{g}$-module by the action $m x=-x m$ for $m \in M, x \in \mathfrak{g}$.

Next consider the graded dual $M^{*}=\bigoplus_{n}\left(M^{*}\right)_{n}$ as a right module by the action $(f X)(m)=f(X m)$. Let

$$
\Psi: U^{*} \otimes_{\mathbb{C}} M \stackrel{\sim}{\rightarrow} U^{*} \otimes_{\mathbb{C}} M
$$

be the linear isomorphism defined by $\Psi(f \otimes m)(u \otimes g)=(f \otimes m)((1 \otimes g) \Delta(u))$ for $f \in U^{*}, m \in M, u \in U, g \in M^{*}$, where $M$ is identified with $\left(M^{*}\right)^{*}$. Extend this to the linear isomorphism

$$
\tilde{\Phi}_{2}: U^{*} \otimes_{\mathbb{C}} U \otimes_{\mathbb{C}} M \stackrel{\sim}{\rightarrow} U^{*} \otimes_{\mathbb{C}} U \otimes_{\mathbb{C}} M
$$

by setting $\tilde{\Phi}_{2}(f \otimes u \otimes m)=\sum_{i} f_{i} \otimes u \otimes m_{i}$ if $\Psi(f \otimes m)=\sum_{i} f_{i} \otimes m_{i}$ with $f_{i} \in U^{*}$, $m_{i} \in M$. Then

$$
\begin{aligned}
& \tilde{\Phi}_{2} \circ \pi_{1, R}(X)=\left(\pi_{1, R}(X)+\pi_{3, R}(X)\right) \circ \tilde{\Phi}_{2}, \\
& \tilde{\Phi}_{2} \circ\left(\pi_{1, L}(X)+\pi_{3, L}(X)\right)=\pi_{1, L}(X) \circ \tilde{\Phi}_{2} .
\end{aligned}
$$

Set

$$
\tilde{\Phi}=\tilde{\Phi}_{2} \circ \tilde{\Phi}_{1}: U^{*} \otimes_{\mathbb{C}} U \otimes_{\mathbb{C}} M \stackrel{\sim}{\rightarrow} U^{*} \otimes_{\mathbb{C}} U \otimes_{\mathbb{C}} M
$$


Then

$$
\begin{aligned}
& \tilde{\Phi} \circ\left(\pi_{1, L}(X)+\pi_{2, L}(X)\right)=\tilde{\Phi}_{2} \circ\left(\pi_{1, L}(X)+\pi_{2, L}(X)+\pi_{3, L}(X)\right) \circ \tilde{\Phi}_{1} \\
& =\left(\pi_{1, L}(X)+\pi_{2, L}(X)\right) \circ \tilde{\Phi} \\
& \tilde{\Phi} \circ\left(\pi_{2, R}(X)+\pi_{3, R}(X)\right)=\tilde{\Phi}_{2} \circ \pi_{2, R}(X) \circ \tilde{\Phi}_{1}=\pi_{2, R}(X) \circ \tilde{\Phi}, \\
& \tilde{\Phi} \circ \pi_{1, R}(X)=\tilde{\Phi}_{2} \circ \pi_{1, R}(X) \circ \tilde{\Phi}_{1}=\left(\pi_{1, R}(X)+\pi_{3, R}(X)\right) \circ \tilde{\Phi} .
\end{aligned}
$$

By (9) and the definition of $U S(\mathfrak{g}), \tilde{\Phi}$ gives rise to a linear isomorphism

$$
\Phi: U S(\mathfrak{g}) \otimes_{\mathbb{C}} M \stackrel{\sim}{\rightarrow} U S(\mathfrak{g}) \otimes_{\mathbb{C}} M .
$$

Moreover, $\Phi$ satisfies the required properties by (10) and (11).

2.5. Semi-infinite induction. Let $\mathfrak{h}=\bigoplus_{n \in \mathbb{Z}} \mathfrak{h}_{n}$ be a graded Lie subalgebra of $\mathfrak{g}$ such that $\left.\gamma\right|_{\mathfrak{h}}$ is a semi-infinite 1-cochain of $\mathfrak{h}$. Following Vor2 we define the semi-induced $\mathfrak{g}$-module S-ind $\mathfrak{h}_{\mathfrak{h}}^{\mathfrak{g}} M$ as

$$
\operatorname{S-ind}_{\mathfrak{h}}^{\mathfrak{g}} M:=H^{\frac{\infty}{2}+0}\left(\mathfrak{h}, U S(\mathfrak{g}) \otimes_{\mathbb{C}} M\right),
$$

where $U S(\mathfrak{g}) \otimes_{\mathbb{C}} M$ is considered as an $\mathfrak{h}$-module by the action $\pi_{1}$ defined in (7). The space $\mathrm{S}$-ind $\mathfrak{h}_{\mathfrak{h}}^{\mathfrak{g}} M$ inherits the structure of a $\mathfrak{g}$-module from the action $\pi_{2}$ defined in (7).

Lemma 2.2. The assignment $\mathrm{S}$-ind $\mathrm{h}_{\mathfrak{h}}^{\mathfrak{g}}: M \mapsto \mathrm{S}^{-i n d} \mathrm{~d}_{\mathfrak{h}}^{\mathfrak{g}} M$ defines an exact functor from $\tilde{\mathcal{O}}^{\mathfrak{h}}$ to $\tilde{\mathcal{O}}^{\mathfrak{g}}$.

Proof. Clearly, S-ind $M$ is an object of $\tilde{\mathcal{O}}^{\mathfrak{g}}$ since $U S(\mathfrak{g}) \otimes_{\mathbb{C}} M$ is. By Proposition 2.1 we may replace the actions of $\pi_{1}$ and $\pi_{2}$ on $U S(\mathfrak{g}) \otimes_{\mathbb{C}} M$ with $\pi_{1}^{\prime}$ and $\pi_{2}^{\prime}$, simultaneously. It follows that

$$
H^{\frac{\infty}{2}+\bullet}\left(\mathfrak{h}, U S(\mathfrak{g}) \otimes_{\mathbb{C}} M\right) \cong H^{\frac{\infty}{2}+\bullet}(\mathfrak{h}, U S(\mathfrak{g})) \otimes_{\mathbb{C}} M .
$$

Since $U S(\mathfrak{g})$ is free over $\mathfrak{h}_{-}$and cofree over $\mathfrak{h}_{+}, H^{\frac{\infty}{2}}+i(\mathfrak{h}, U S(\mathfrak{g}))=0$ for $i \neq 0$ by [Vor1, Theorem 2.1]. (Note that the spectral sequence on Vor1] converges since the complex $U S(\mathfrak{g}) \otimes \bigwedge^{\frac{\infty}{2}+\bullet}(\mathfrak{h})$ is a direct sum of finite-dimensional subcomplexes consisting of homogeneous vectors.) We have shown that the functor $\mathrm{S}_{-} \mathrm{ind}_{\mathfrak{h}}^{\mathfrak{g}}$ is exact.

In the case that $\mathfrak{h}=\mathfrak{g}$ and $\gamma_{0}=\gamma$, we have the following assertion.

Proposition 2.3 ([Vor2, (1.9)]). The functor $\mathrm{S} \operatorname{ind}_{\mathfrak{g}}^{\mathfrak{g}}: \tilde{\mathcal{O}}^{\mathfrak{g}} \rightarrow \tilde{\mathcal{O}}^{\mathfrak{g}}$ is isomorphic to the identify functor.

Proof. As $H^{\frac{\infty}{2}+0}(\mathfrak{g}, U S(\mathfrak{g}))$ is isomorphic to the trivial representation $\mathbb{C}$ of $\mathfrak{g}$ ( Vor 1 , Theorem 2.1]), (12) gives the $\mathfrak{g}$-module isomorphism $\mathrm{S}_{\text {-ind }} \mathfrak{g} M \cong M$ as required.

2.6. Suppose that $\mathfrak{g}$ admits a decomposition

$$
\mathfrak{g}=\mathfrak{a} \oplus \overline{\mathfrak{a}}
$$

with graded subalgebras $\mathfrak{a}$ and $\overline{\mathfrak{a}}$ such that the restrictions $\left.\gamma\right|_{\mathfrak{a}}$ and $\left.\gamma\right|_{\overline{\mathfrak{a}}}$ of $\gamma$ are semi-infinite 1-cochains of $\mathfrak{a}$ and $\overline{\mathfrak{a}}$, respectively.

Lemma 2.4. $U S(\mathfrak{g}) \cong U S(\mathfrak{a}) \otimes_{\mathbb{C}} U S(\overline{\mathfrak{a}})$ as left $\mathfrak{a}$-modules and right $\overline{\mathfrak{a}}$-modules. 
Proof. We have $U_{+}^{*} \cong U\left(\mathfrak{a}_{+}\right)^{*} \otimes_{\mathbb{C}} U\left(\overline{\mathfrak{a}}_{+}\right)^{*}$ as left $\mathfrak{a}_{+}$-modules and right $\overline{\mathfrak{a}}_{+}$-modules. Consider the composition

$$
\begin{aligned}
U S(\mathfrak{a}) \otimes_{\mathbb{C}} U S(\overline{\mathfrak{a}}) \stackrel{\sim}{\rightarrow}\left(U\left(\mathfrak{a}_{-}\right) \otimes_{\mathbb{C}} U\left(\mathfrak{a}_{+}\right)^{*}\right) \otimes_{\mathbb{C}}\left(U\left(\overline{\mathfrak{a}}_{+}\right)^{*} \otimes_{\mathbb{C}} U\left(\overline{\mathfrak{a}}_{-}\right)\right) \\
\stackrel{\sim}{\rightarrow} U\left(\mathfrak{a}_{+}\right) \otimes_{\mathbb{C}} U_{+}^{*} \otimes_{\mathbb{C}} U\left(\overline{\mathfrak{a}}_{+}\right) \rightarrow U S(\mathfrak{g}),
\end{aligned}
$$

where the last map is the multiplication map. From the description (5), (6) of the $\mathfrak{g}$-bimodule structure of semi-regular bimodules one sees that the image of the above map is stable under the left and the right action of $\mathfrak{g}$ on $U S(\mathfrak{g})$. Hence the image must coincide with $U S(\mathfrak{g})$ since it contains $U_{+}^{*}$. By the equality of the graded dimensions it follows that the above map is an isomorphism.

Lemma 2.5. For $M \in \tilde{\mathcal{O}}^{\overline{\mathfrak{a}}}, \operatorname{S-ind}_{\overline{\mathfrak{a}}}^{\mathfrak{g}} M \cong U S(\mathfrak{a}) \otimes_{\mathbb{C}} M$ as $\mathfrak{a}$-modules, where $\mathfrak{a}$ acts only on the first factor $U S(\mathfrak{a})$ of $U S(\mathfrak{a}) \otimes_{\mathbb{C}} M$.

Proof. We have

$$
\begin{array}{r}
\operatorname{S-ind}_{\overline{\mathfrak{a}}}^{\mathfrak{g}}(M) \cong H^{\frac{\infty}{2}+0}\left(\overline{\mathfrak{a}}, U S(\mathfrak{a}) \otimes_{\mathbb{C}} U S(\overline{\mathfrak{a}}) \otimes_{\mathbb{C}} M\right) \\
\cong U S(\mathfrak{a}) \otimes_{\mathbb{C}} \operatorname{S}_{-i n d} \overline{\overline{\mathfrak{a}}}(M) \cong U S(\mathfrak{a}) \otimes_{\mathbb{C}} M
\end{array}
$$

by Lemmas 2.3 and 2.4

\section{Semi-Infinite Bruhat ordering}

3.1. Affine Kac-Moody algebras and affine Weyl groups. We first fix some notation which are used for the rest of the paper.

Let $\stackrel{\circ}{\mathfrak{g}}$ be a finite-dimensional complex simple Lie algebra, and fix a Cartan subalgebra $\stackrel{\circ}{\mathfrak{h}}$ of $\stackrel{\circ}{\mathfrak{g}}$. Let $\stackrel{\circ}{\Delta} \subset \stackrel{\circ}{\mathfrak{h}}^{*}$ be the set of roots of $\stackrel{\circ}{\mathfrak{g}}$. Choose a subset $\Delta_{+} \subset \stackrel{\circ}{\mathfrak{h}}^{*}$ of positive roots and the set $\stackrel{\circ}{\Pi}=\left\{\alpha_{i} ; i \in \stackrel{\circ}{I}\right\} \subset \Delta_{+}, \stackrel{\circ}{I}=\{1,2, \ldots, l\}$, of simple roots. Let $\theta$ be the highest root, $\theta_{s}$ the highest short root, $\Delta_{-}=-\Delta_{+}, \stackrel{\circ}{\rho}=\frac{1}{2} \sum_{\alpha \in \Delta_{+}} \alpha$.

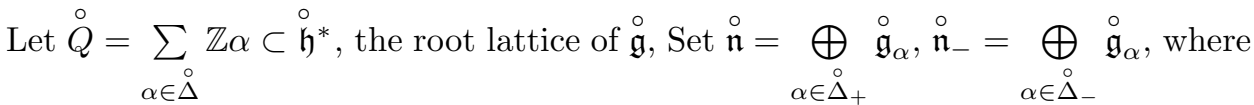
$\stackrel{\circ}{\mathfrak{g}}_{\alpha}$ is the root space of $\stackrel{\circ}{\mathfrak{g}}$ with root $\alpha$. We have the triangular decomposition

$$
\stackrel{\circ}{\mathfrak{g}}=\stackrel{\circ}{\mathfrak{n}}_{-} \oplus \stackrel{\circ}{\mathfrak{h}} \oplus \stackrel{\circ}{\mathfrak{n}} .
$$

Let $(\mid$ ) be the normalized invariant bilinear form of $\stackrel{\circ}{\mathfrak{g}}$. We identify $\stackrel{\circ}{\mathfrak{h}}$ with $\stackrel{\circ}{\mathfrak{h}}$ * using $(\mid)$. Let $\stackrel{\circ}{\Delta} \vee=\left\{\alpha^{\vee}: \alpha \in \stackrel{\circ}{\Delta}\right\}$, the set of coroots, $\stackrel{\circ}{Q}^{\vee}=\sum_{\alpha \in \Delta} \mathbb{Z} \alpha^{\vee} \subset \grave{h}=\stackrel{\circ}{\mathfrak{h}}^{*}$, the coroot lattice of $\stackrel{\circ}{\mathfrak{g}}, \stackrel{\circ}{\rho} \rho^{\vee}=\frac{1}{2} \sum_{\substack{\alpha \in \Delta_{+}\\}} \alpha^{\vee}$, where $\alpha^{\vee}=2 \alpha /(\alpha \mid \alpha)$.

Let $\stackrel{\circ}{\mathcal{W}} \subset G L\left(\stackrel{\circ}{\mathfrak{h}}^{*}\right)$ be the Weyl group of $\stackrel{\circ}{\mathfrak{g}}$, and let $s_{\alpha} \in \stackrel{\circ}{\mathcal{W}}$ be the reflection corresponding to $\alpha \in \Delta: s_{\alpha}(\lambda)=\lambda-\lambda\left(\alpha^{\vee}\right) \alpha$.

Let $\mathfrak{g}$ be the affine Kac-Moody algebra associated with $\stackrel{\circ}{\mathfrak{g}}$ :

$$
\mathfrak{g}=\stackrel{\circ}{\mathfrak{g}}\left[t, t^{-1}\right] \oplus \mathbb{C} K \oplus \mathbb{C} D .
$$

The commutation relations of $\mathfrak{g}$ are given by

$$
\left[x t^{m}, y t^{n}\right]=[x, y] t^{m+n}+m \delta_{m+n, 0}(x \mid y) K, \quad[K, \mathfrak{g}]=0, \quad\left[D, x t^{n}\right]=n x t^{n} .
$$


We consider $\stackrel{\circ}{\mathfrak{g}}$ as a subalgebra of $\mathfrak{g}$ by the natural embedding $\stackrel{\circ}{\mathfrak{g}} \hookrightarrow \mathfrak{g}, x \mapsto x t^{0}$. Let

$$
\mathfrak{h}=\grave{h} \oplus \mathbb{C} K \oplus \mathbb{C} D,
$$

be the Cartan subalgebra of $\mathfrak{g}$. The bilinear form $(\mid)$ from $\stackrel{\circ}{\mathfrak{h}}$ to $\mathfrak{h}$ by letting $(K \mid \mathfrak{\circ})=$ $(D \mid \mathfrak{h})=(K \mid K)=(D \mid D)=0$ and $(D \mid K)=1$. We identify $\stackrel{\circ}{\mathfrak{h}}^{*}$ with the subspace of $\mathfrak{h}^{*}$ consisting of elements which vanish on $\mathbb{C} K \oplus \mathbb{C} D$. Thus,

$$
\mathfrak{h}^{*}=\stackrel{\circ}{\mathfrak{h}}^{*} \oplus \mathbb{C} \Lambda_{0} \oplus \mathbb{C} \delta,
$$

where $\Lambda_{0}$ and $\delta$ are defined by $\Lambda_{0}(K)=\delta(D)=1, \Lambda_{0}(\stackrel{\circ}{\mathfrak{h}} \oplus \mathbb{C} \delta)=\delta(\stackrel{\circ}{\mathfrak{h}} \oplus \mathbb{C} K)=0$. The number $\langle\lambda, K\rangle$ is called the level of $\lambda$.

Let $\Delta^{r e}=\stackrel{\circ}{\Delta_{+}} \sqcup\{\alpha+n \delta ; \alpha \in \stackrel{\circ}{\Delta}, n \in \mathbb{N}\}$, the set of positive real roots of $\mathfrak{g}, \Delta_{-}^{r e}=-\Delta_{+}^{r e}, \Delta^{r e}=\Delta_{+}^{r e} \sqcup \Delta_{-}^{r e}$ the set of real roots, $\Pi=\left\{\alpha_{0}=-\theta+\delta, \alpha_{1}, \ldots, \alpha_{\ell}\right\}$ the set of simple roots.

Let $\mathcal{W}$ be the Weyl group of $\mathfrak{g}$, or the affine Weyl group of $\stackrel{\circ}{\mathcal{W}}$. We have

$$
\mathcal{W}=\stackrel{\circ}{\mathcal{W}} \ltimes \stackrel{\circ}{Q^{\vee}} \text {. }
$$

The extended affine Weyl group $\mathcal{W}^{e}$ of $\mathfrak{g}$ is the semidirect product

$$
\mathcal{W}^{e}=\stackrel{\circ}{\mathcal{W}} \ltimes P^{\vee}
$$

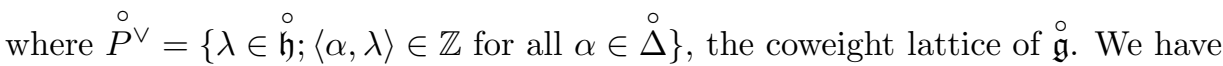

$$
\mathcal{W}^{e}=\mathcal{W}_{+}^{e} \ltimes \mathcal{W},
$$

where $\mathcal{W}_{+}^{e}$ is the subgroup of $\mathcal{W}^{e}$ consisting of elements which fix the set $\Pi$.

We denote by $t_{\alpha}$ or simply by $\alpha$ for the element of $\mathcal{W}^{e}$ corresponding to $\alpha \in \stackrel{\circ}{P^{\vee}}$. The reflection $s_{\alpha}$ corresponding to $\alpha=\bar{\alpha}+n \delta \in \Delta^{r e}$ is given by $s_{\alpha}=t_{-n \bar{\alpha}^{\vee}} s_{\bar{\alpha}}$. We set $s_{i}=s_{\alpha_{i}}$ for $i \in I:=\{0,1, \ldots, l\}$, so that $\mathcal{W}=\left\langle s_{i} ; i \in I\right\rangle$. The action of $\mathcal{W}$ on $\mathfrak{h}^{*}$ is extended to the action of $\mathcal{W}^{e}$ on $\mathfrak{h}^{*}$ by

$$
\begin{aligned}
& w\left(\Lambda_{0}\right)=\Lambda_{0}, w(\delta)=\delta \quad w \in \stackrel{\circ}{\mathcal{W}}, \\
& t_{\alpha}(\lambda)=\lambda+\langle\Lambda, K\rangle \alpha-\left(\langle\lambda, \alpha\rangle+\frac{(\alpha \mid \alpha)}{2}\langle\lambda, K\rangle\right) \delta, \quad \lambda \in \mathfrak{h}^{*} .
\end{aligned}
$$

For $\lambda \in \tilde{\mathfrak{h}}^{*}$ let $\bar{\lambda} \in \stackrel{\circ}{\mathfrak{h}}^{*}$ be its restriction to $\stackrel{\circ}{\mathfrak{h}}$.

3.2. Twisted Bruhat ordering. Let $\ell: \mathcal{W}^{e} \rightarrow \mathbb{Z}_{\geq 0}$ be the length function: $\ell(w)=\sharp\left(\Delta_{+}^{r e} \cap w\left(\Delta_{-}^{r e}\right)\right)$. We have

$$
\ell\left(t_{\mu} y\right)=\sum_{\alpha \in \Delta_{+} \cap y\left(\Delta_{+}\right)}|(\alpha \mid \mu)|+\sum_{\alpha \in \Delta_{+} \cap y\left(\Delta_{-}\right)}|1-(\alpha \mid \mu)|
$$

for $\mu \in \stackrel{\circ}{P}^{\vee}, y \in \stackrel{\circ}{\mathcal{W}}$. by

The twisted length function Ark1] $\ell^{y}: \mathcal{W}^{e} \rightarrow \mathbb{Z}$ with the twist $y \in \mathcal{W}^{e}$ is defined

$$
\ell^{y}(w)=\sharp\left(\Delta_{+}^{r e} \cap w\left(\Delta_{-}^{r e}\right) \cap y\left(\Delta_{+}^{r e}\right)\right)-\sharp\left(\Delta_{+}^{r e} \cap w\left(\Delta_{-}^{r e}\right) \cap y\left(\Delta_{-}^{r e}\right)\right) .
$$


Lemma 3.1. Let $w, y \in \mathcal{W}^{e}$.

(i) Suppose that $\ell\left(y s_{i}\right)=\ell(y)+1$ for $i \in I$. Then

$$
\ell^{y s_{i}}(w)= \begin{cases}\ell^{y}(w) & \text { if } w^{-1} y\left(\alpha_{i}\right) \in \Delta_{+}^{r e}, \\ \ell^{y}(w)-2 & \text { if } w^{-1} y\left(\alpha_{i}\right) \in \Delta_{-}^{r e} .\end{cases}
$$

(ii) $\ell^{y}(w)=\ell\left(y^{-1} w\right)-\ell\left(y^{-1}\right)$.

Proof. (i) The assertion follows from the definition and the fact that

$$
\Delta_{+}^{r e} \cap y s_{i}\left(\Delta_{-}^{r e}\right)=\Delta_{+}^{r e} \cap y\left(\Delta_{-}^{r e}\right) \sqcup\left\{y\left(\alpha_{i}\right)\right\} \quad \text { if } \ell\left(y s_{i}\right)=\ell(y)+1 .
$$

(ii) We prove by induction on $\ell(y)$. If $\ell(y)=0$, then $\ell^{y}(w)=\ell(w)=\ell\left(y^{-1} w\right)$. Suppose that $\ell\left(y s_{i}\right)=\ell(y)+1$. If $w^{-1} y\left(\alpha_{i}\right) \in \Delta_{+}^{r e}$, then $\ell\left(s_{i} y^{-1} w\right)=\ell\left(y^{-1} w\right)+1$. Hence by (i) and induction hypothesis,

$$
\ell^{y s_{i}}(w)=\ell^{y}(w)=\ell\left(y^{-1} w\right)-\ell\left(y^{-1}\right)=\ell\left(s_{i} y^{-1} w\right)-\ell\left(s_{i} y^{-1}\right) .
$$

If $w^{-1} y\left(\alpha_{i}\right) \in \Delta_{-}^{r e}$, then $\ell\left(s_{i} y^{-1} w\right)=\ell\left(y^{-1} w\right)-1$. Again by (i) and induction hypothesis,

$$
\ell^{y s_{i}}(w)=\ell^{y}(w)-2=\ell\left(y^{-1} w\right)-2-\ell\left(y^{-1}\right)=\ell\left(s_{i} y^{-1} w\right)-\ell\left(s_{i} y^{-1}\right) .
$$

This completes the proof.

For $w_{1}, w_{2}, y \in \mathcal{W}$ and $\gamma \in \Delta^{r e}$, write $w_{1} \underset{y}{\stackrel{\gamma}{\longrightarrow}} w_{2}$ if $w_{1}=s_{\gamma} w_{2}$ and $\ell^{y}\left(w_{1}\right)>$ $\ell^{y}\left(w_{2}\right)$. Below, we shall often omit the symbol $\gamma$ above the arrow. Also, we shall omit the symbol $y$ under the arrow if $y=1$. By Lemma 3.1(ii) we have

$$
w_{1} \underset{y}{\stackrel{y(\gamma)}{\longrightarrow}} w_{2} \quad \Longleftrightarrow \quad y^{-1} w_{1} \stackrel{\gamma}{\longrightarrow} y^{-1} w_{2} \text {. }
$$

In particular for $\beta=y\left(\alpha_{i}\right) \in \Delta_{+}^{r e}, \alpha_{i} \in \Pi$, and $w_{1}, w_{2} \in \mathcal{W}$ such that $\ell^{y}\left(w_{2}\right)-$ $\ell^{y}\left(w_{1}\right)=1$ we have the equivalence
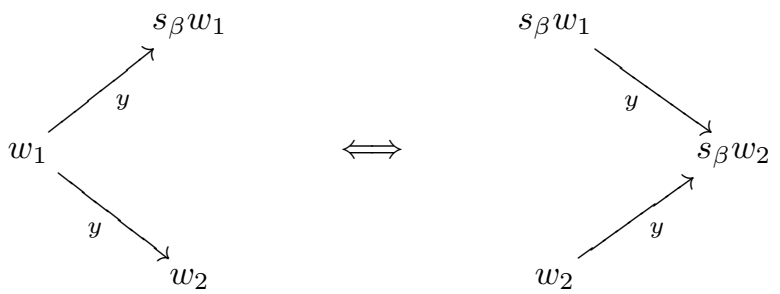

by BGG, Lemma 11.3].

Define $w \succeq_{y} w^{\prime}$ if there exists a sequence $w_{1}, w_{2}, \ldots, w_{k}$ of elements of $\mathcal{W}$ such that

$$
w \underset{y}{\longrightarrow} w_{1} \underset{y}{\longrightarrow} w_{2} \underset{y}{\longrightarrow} \ldots \underset{y}{\longrightarrow} w_{k} \underset{y}{\longrightarrow} w^{\prime} .
$$

Note that

$$
w \succeq_{y} w^{\prime} \Longleftrightarrow y^{-1} w \succeq y^{-1} w^{\prime},
$$

by (14), where $\succeq=\succeq_{1}$, the usual Bruhat ordering of $\mathcal{W}$. It follows that $\succeq_{y}$ defines a partial ordering of $\mathcal{W}$.

We will use the symbol $w \triangleright_{y} w^{\prime}$ to denote a covering in the twisted Bruhat order $\succeq_{y}$. Thus $w \triangleright_{y} w^{\prime}$ means that $w \succeq_{y} w^{\prime}$ and $\ell^{y}(w)=\ell^{y}\left(w^{\prime}\right)+1$. 
3.3. Semi-infinite Bruhat ordering. Define the semi-infinite length FF2 $\ell^{\frac{\infty}{2}}(w)$ of $w \in \mathcal{W}^{e}$ by

$$
\ell^{\frac{\infty}{2}}(w)=\sharp\left\{\alpha \in \Delta_{+}^{r e} \cap w\left(\Delta_{-}^{r e}\right) ; \bar{\alpha} \in \stackrel{\circ}{\Delta}_{+}\right\}-\sharp\left\{\alpha \in \Delta_{+}^{r e} \cap w\left(\Delta_{-}^{r e}\right) ; \bar{\alpha} \in \stackrel{\circ}{\Delta}_{-}\right\} .
$$

We have

$$
\ell^{\frac{\infty}{2}}\left(t_{\lambda} y\right)=\ell(y)-2(\stackrel{\circ}{\rho} \mid \lambda)
$$

for $\lambda \in \stackrel{\circ}{P}^{\vee}, w \in \stackrel{\circ}{\mathcal{W}}$.

Set

$$
\stackrel{\circ}{P_{+}^{\vee}}=\left\{\lambda \in \stackrel{\circ}{P^{\vee}} ; \alpha(\lambda) \geq 0 \text { for all } \alpha \in \stackrel{\circ}{\Delta}_{+}\right\},
$$

We say that $\lambda \in \stackrel{\circ}{P_{+}^{\vee}}$ is sufficiently large if $\alpha(\lambda)$ is sufficiently large for all $\alpha \in \stackrel{\circ}{\Delta_{+}}$.

By (13) and (17) we have the following assertion.

Lemma 3.2. $\ell^{\frac{\infty}{2}}(w)=\ell^{\lambda}(w)=-\ell^{-\lambda}(w)$ for a sufficiently large $\lambda \in \stackrel{\circ}{P_{+}^{\vee}}$.

We write

$$
w_{1} \underset{\frac{\infty}{2}}{\stackrel{\gamma}{\longrightarrow}} w_{2}
$$

for $w_{1}, w_{2} \in \mathcal{W}$ and $\gamma \in \Delta^{r e}$ if $w_{1}=w_{2} s_{\gamma}$ and $\ell^{\frac{\infty}{2}}\left(w_{1}\right)<\ell^{\frac{\infty}{2}}\left(w_{2}\right)$. (We shall often omit the symbol $\gamma$ above the arrow.) Define $w \succeq \frac{\infty}{2} w^{\prime}$ if there exists a sequence $w_{1}, w_{2}, \ldots, w_{k}$ of elements of $\mathcal{W}$ such that

By Lemma 3.2

$$
w \underset{\frac{\infty}{2}}{\longrightarrow} w_{1} \underset{\frac{\infty}{2}}{\longrightarrow} w_{2} \underset{\frac{\infty}{2}}{\longrightarrow} \ldots \underset{\frac{\infty}{2}}{\longrightarrow} w_{k} \underset{\frac{\infty}{2}}{\longrightarrow} w^{\prime} .
$$

$$
\begin{aligned}
w \succeq_{\frac{\infty}{2}} w^{\prime} & \Longleftrightarrow w^{\prime} \succeq_{t_{\lambda}} w \quad \text { for a sufficiently large } \lambda \in \stackrel{\circ}{P}_{+}^{\vee}, \\
& \Longleftrightarrow w \succeq_{t_{-\lambda}} w^{\prime} \quad \text { for a sufficiently large } \lambda \in \stackrel{\circ}{P_{+}^{\vee}} .
\end{aligned}
$$

It follows that $\succeq_{\frac{\infty}{2}}$ defines a partial ordering of $\mathcal{W}$. Following Arkhipov Ark1, we call it the semi-infinite Bruhat ordering on $\mathcal{W}$. By [Soe1, Claim 4.14] the semi-infinite Bruhat ordering coincides with the generic Bruhat ordering defined by Lusztig Lus.

We will use the symbol $w \triangleright \frac{\infty}{2} w^{\prime}$ to denote a covering in the twisted Bruhat order $\succeq \frac{\infty}{2}$. Thus $w \triangleright_{\frac{\infty}{2}} w^{\prime}$ means that $w \succeq \frac{\infty}{2} w^{\prime}$ and $\ell^{\frac{\infty}{2}}(w)=\ell^{\frac{\infty}{2}}\left(w^{\prime}\right)-1$.

\subsection{Semi-infinite analogue of parabolic subgroups and minimal (maxi-} mal) length representatives. Let $S$ be a subset of $\stackrel{\circ}{\Pi}, \stackrel{\circ}{\Delta}_{S}$ the subroot system of $\stackrel{\circ}{\Delta}$ generated by $\alpha_{i} \in S, \stackrel{\circ}{\Delta}_{S}=\bigsqcup_{i=1}^{r} \stackrel{\circ}{\Delta}_{S, i}$ the decomposition into the simple subroot systems $\stackrel{\circ}{\Delta}_{1, S}, \ldots, \stackrel{\circ}{\Delta}_{r, S}$. Let $\theta_{i}$ be the longest root of $\stackrel{\circ}{\Delta}_{S, i}$.

Set

$$
\Delta_{S}=\left\{\alpha+n \delta \in \Delta^{r e} ; \alpha \in \stackrel{\circ}{\Delta}_{S}, n \in \mathbb{Z}\right\}, \quad \mathcal{W}_{S}=\left\langle s_{\alpha} ; \alpha \in \Delta_{S}\right\rangle \subset \mathcal{W} .
$$

Then $\Delta_{S}$ is a subroot system of $\Delta^{r e}$ isomorphic to the affine root system associated with $\stackrel{\circ}{\Delta}_{S}$. Put $\Delta_{S,+}=\Delta_{S} \cap \Delta_{+}^{r e}$, the set of positive root of $\Delta_{S}$. Then $\Pi_{S}=$ $S \sqcup\left\{-\theta_{1}+\delta, \ldots,-\theta_{s}+\delta\right\}$ is a set of simple roots of $\Delta_{S}$. We have $\mathcal{W}_{S}=\stackrel{\circ}{\mathcal{W}}_{S} \ltimes t_{Q^{\vee}}$, 
where $\stackrel{\circ}{Q_{S}^{\vee}}=\sum_{\alpha \in \searrow_{S}} \mathbb{Z} \alpha^{\vee}$. By (17), the restriction of the semi-infinite length function to $\mathcal{W}_{S}$ coincides with the semi-infinite length function of the affine Weyl group $\mathcal{W}_{S}$.

Define

$$
\mathcal{W}^{S}=\left\{w \in \mathcal{W} ; w^{-1}\left(\Delta_{S,+}\right) \subset \Delta_{+}^{r e}\right\} .
$$

Theorem 3.3 ([et]). The multiplication map $\mathcal{W}_{S} \times \mathcal{W}^{S} \rightarrow \mathcal{W},(u, v) \mapsto u v$, is a bijection. Moreover, we have

$$
\ell^{\frac{\infty}{2}}(u v)=\ell^{\frac{\infty}{2}}(u)+\ell^{\frac{\infty}{2}}(v) \quad \text { for } u \in \mathcal{W}_{S}, v \in \mathcal{W}^{S} .
$$

Proof. First, we show the injectivity of the multiplication map. Suppose that $u_{1} v_{1}=u_{2} v_{2}$ with $u_{i} \in \mathcal{W}_{S}, v_{i} \in \mathcal{W}^{S}$. Then $v_{1}=u v_{2}$ with $u=u_{1}^{-1} u_{2} \in \mathcal{W}_{S}$. If $u \neq 1$, then there exists $\alpha \in \Delta_{S,+}$ such that $u^{-1}(\alpha) \in-\Delta_{S,+}$. But then $v_{2} \in \mathcal{W}^{S}$ implies that $v_{1}^{-1}(\alpha)=v_{2}^{-1} u^{-1}(\alpha) \in \Delta_{-}^{r e}$, and this contradicts that $v_{1} \in \mathcal{W}^{S}$. Hence $u_{1}=u_{2}$, and so $v_{1}=v_{2}$.

Second, we show that the multiplication map $\mathcal{W}_{S} \times \mathcal{W}^{S} \rightarrow \mathcal{W}$ is surjective. We will prove by induction on $\sharp\left(w^{-1}\left(\Delta_{S,+}\right) \cap \Delta_{-}^{r e}\right)$ that there exists $u \in \mathcal{W}_{S}$ such that $u^{-1} w \in \mathcal{W}^{S}$. If $\sharp\left(w^{-1}\left(\Delta_{S,+}\right) \cap \Delta_{-}^{r e}\right)=0, w \in \mathcal{W}^{S}$ there is nothing to show. Suppose that $\sharp\left(w^{-1}\left(\Delta_{S,+}\right) \cap \Delta_{-}^{r e}\right)>0$. Then there exists $\beta \in \Pi_{S}$ such that $w^{-1}(\beta) \in \Delta_{-}^{r e}$. Indeed, any element $\alpha \in \Delta_{S,+}$ is expressed as $\alpha=\sum_{\beta \in \Pi_{S}} n_{\beta} \beta$ with $n_{\beta} \in \mathbb{Z}_{\geq 0}$. Thus $w^{-1}(\alpha)=\sum_{\beta \in \Pi_{S}} n_{\beta} w^{-1}(\beta) \in \Delta_{-}^{r e}$ implies that one of $w^{-1}(\beta)$ must belong to $\Delta_{-}^{r e}$. Now because $\left(s_{\beta} w\right)^{-1}\left(\Delta_{S,+}\right)=w^{-1} s_{\beta}\left(\Delta_{S,+}\right)=w^{-1}\left(\Delta_{S,+} \backslash\{\beta\} \sqcup\{-\beta\}\right)=$ $w^{-1}\left(\Delta_{S,+}\right) \backslash\left\{w^{-1}(\beta)\right\} \sqcup\left\{-w^{-1}(\beta)\right\}$,

$$
\left(s_{\beta} w\right)^{-1}\left(\Delta_{S,+}\right) \cap \Delta_{-}^{r e}=w^{-1}\left(\Delta_{S,+}\right) \cap \Delta_{-}^{r e} \backslash\left\{w^{-1}(\beta)\right\} .
$$

Hence by applying the induction hypothesis to $s_{\beta} w$ we find an element $u \in \mathcal{W}_{S}$ such that $u^{-1} s_{\beta} w \in \mathcal{W}^{S}$.

Finally, we prove the equality of the semi-infinite length. By (17), we have $\ell^{\frac{\infty}{2}}\left(t_{\mu} w\right)=\ell^{\frac{\infty}{2}}\left(t_{\mu}\right)+\ell^{\frac{\infty}{2}}(w)$ for any $\mu \in \stackrel{\circ}{Q^{\vee}}$. Hence we may assume that $u \in \stackrel{\circ}{\mathcal{W}}_{S}$. We will prove by induction on the length $\ell(u)$ of $u \in \mathcal{W}_{S}$ that $\ell^{\frac{\infty}{2}}(u v)=\ell^{\frac{\infty}{2}}(u)+$ $\ell^{\frac{\infty}{2}}(v)$ for any $v \in \mathcal{W}^{S}$. Suppose that $\ell(u)=1$, so that $u=s_{i}$ for some $\alpha_{i} \in S$. Let $v=t_{\mu} y \in \mathcal{W}^{S}$ with $\mu \in \stackrel{\circ}{Q^{\vee}}, y \in \stackrel{\circ}{\mathcal{W}}$. Note that $v \in \mathcal{W}^{S}$ is equivalent to:

$$
\text { if } \alpha \in \stackrel{\circ}{\Delta}_{S,+} \text { then } \alpha(\mu)= \begin{cases}0 & \text { if } y^{-1}(\alpha) \in \stackrel{\circ}{\Delta}_{+}, \\ 1 & \text { if } y^{-1}(\alpha) \in \stackrel{\circ}{\Delta}_{-} .\end{cases}
$$

Since

$$
\ell^{\frac{\infty}{2}}\left(s_{i} t_{\mu} y\right)=\ell\left(t_{s_{i}(\mu)} s_{i} y\right)=\ell\left(s_{i} y\right)-2\left(\rho \mid \mu-\alpha_{i}(\mu) \alpha_{i}^{\vee}\right)=\ell\left(s_{i} y\right)-2(\rho \mid \mu)+2 \alpha_{i}(\mu),
$$

(18) implies that $\ell^{\frac{\infty}{2}}\left(s_{i} v\right)=\ell^{\frac{\infty}{2}}(v)+1$. Next let $u=s_{i} u_{1} \in \stackrel{\circ}{\mathcal{W}}_{S}$ with $u_{1} \in \stackrel{\circ}{\mathcal{W}}_{S}$, $\alpha_{i} \in S, \ell(u)=\ell\left(u_{1}\right)+1$, so that $u_{1}^{-1}\left(\alpha_{i}\right) \in \stackrel{\circ}{\Delta}_{+}$. Let $v=t_{\mu} y \in \mathcal{W}^{S}$ as above. We have

$$
\ell^{\frac{\infty}{2}}(u v)=\ell^{\frac{\infty}{2}}\left(t_{s_{i} u_{1}(\mu)} s_{i} u_{1} y\right)=\ell\left(s_{i} u_{1} y\right)-2\left(\rho \mid s_{i} u_{1}(\mu)\right) .
$$

If $\ell\left(s_{i} u_{1} y\right)=\ell\left(u_{1} y\right)+1$, then $\stackrel{\circ}{\Delta}_{+} \ni\left(u_{1} y\right)^{-1}\left(\alpha_{i}\right)=y^{-1}\left(u_{1}^{-1}\left(\alpha_{i}\right)\right)$. Hence $\left(\mu \mid u_{1}^{-1}\left(\alpha_{i}\right)\right)$ $=0$ by (18), which means $s_{i} u_{1}(\mu)=u_{1}(\mu)$. By the induction hypothesis, this proves 
that $\ell^{\frac{\infty}{2}}(u v)=\ell^{\frac{\infty}{2}}(u)+\ell^{\frac{\infty}{2}}(v)$. If $\ell\left(s_{i} u_{1} y\right)=\ell\left(u_{1} y\right)-1$, then $\stackrel{\circ}{\Delta}_{-} \ni\left(u_{1} y\right)^{-1}\left(\alpha_{i}\right)=$ $y^{-1}\left(u_{1}^{-1}\left(\alpha_{i}\right)\right)$. So (18) gives $\left(\mu \mid u_{1}^{-1}\left(\alpha_{i}\right)\right)=1$, which means $s_{i} u_{1}(\mu)=u_{1}(\mu)-\alpha_{i}^{\mathrm{V}}$. By the induction hypothesis, this proves that $\ell^{\frac{\infty}{2}}(u v)=\ell^{\frac{\infty}{2}}(u)+\ell^{\frac{\infty}{2}}(v)$ as required.

\section{WAKImoto modules AND TWISTED VeRma modules}

4.1. The category $\mathcal{O}$ of $\mathfrak{g}$. For any $\mathfrak{h}$-module $M$ we set $M_{\mu}=\{m \in M ; h m=$ $\mu(h) m$ for all $h \in \mathfrak{h}\}$.

Let $\mathcal{O}^{\mathfrak{g}}$ be the full subcategory of $\tilde{\mathcal{O}}^{\mathfrak{g}}$ consisting of modules on which $\mathfrak{h}$ acts semisimply. The formal character of $M \in \mathcal{O}^{\mathfrak{g}}$ is defined by

$$
\operatorname{ch} M=\sum_{\mu \in \mathfrak{h}^{*}}\left(\operatorname{dim}_{\mathbb{C}} M_{\mu}\right) e^{\mu} .
$$

Let $\mathcal{O}_{k}^{\mathfrak{g}}$ be the full subcategory of $\mathcal{O}^{\mathfrak{g}}$ consisting of objects of level $k$, where a $\mathfrak{g}$-module $M$ is said to be of level $k$ if $K$ acts as the multiplication by $k$.

4.2. Twisting functors and twisted Verma modules. By abuse of notation we denote also by $w$ a Tits lifting of $w \in \mathcal{W}^{e}$ to $\operatorname{Aut}(\mathfrak{g})$.

For each $w \in \mathcal{W}$ the twisting functor $T_{w}: \mathcal{O}^{\mathfrak{g}} \rightarrow \mathcal{O}^{\mathfrak{g}}$ is defined as follows ([Ark1]): Let $\mathfrak{n}_{w}=\mathfrak{n}_{-} \cap w^{-1}\left(\mathfrak{n}_{+}\right)$and set $N_{w}=U\left(\mathfrak{n}_{w}\right)$. Put

$$
S_{w}=U \otimes_{N_{w}} N_{w}^{*} .
$$

The space $S_{w}$ has a $U$-bimodule structure, which is described as follows: Let $f \in$ $\mathfrak{n}_{-} \backslash\{0\}$, and set $U_{(f)}=U \otimes_{\mathbb{C}[f]} \mathbb{C}\left[f, f^{-1}\right]$. Then $U_{(f)}$ is an associative algebra which contains $U$ as a subalgebra. We set $S_{f}=U_{(f)} / U$. Choose a filtration $\mathfrak{n}_{w}=F^{0} \supset$ $F^{1} \supset \cdots \supset F^{r} \supset 0, r=\ell(w)$, consisting of ideals $F^{p} \subset \mathfrak{n}_{w}$ of codimension $p$. If $f_{p} \in F^{p-1} \backslash F^{p}$ we have an isomorphism of $U$-bimodules

$$
S_{w}=S_{f_{1}} \otimes_{U} S_{f_{2}} \otimes_{U} \ldots \otimes_{U} S_{f_{r}} .
$$

We have

$$
S_{w} \cong N_{w}^{*} \otimes_{N_{w}} U
$$

as right $U$-modules and left $N_{w}$-modules. Put

$$
\mathbf{1}_{w}^{*}=f_{1}^{-1} \otimes f_{2}^{-1} \otimes \ldots \otimes f_{r}^{-1} \in S_{w} .
$$

For $M \in \mathcal{O}^{\mathfrak{g}}$ define

$$
T_{w}(M)=\phi_{w}\left(S_{w} \otimes_{U(\mathfrak{g})} M\right),
$$

where $\phi_{w}$ means that the action of $\mathfrak{g}$ is twisted by the automorphism $w$ of $\mathfrak{g}$. This defines a right exact functor $T_{w}: \mathcal{O}^{\mathfrak{g}} \rightarrow \mathcal{O}^{\mathfrak{g}}$ such that

$$
T_{w s_{i}} \cong T_{w} T_{i} \quad \text { if } \alpha_{i} \in \Pi \text { and } \ell\left(w s_{i}\right)=\ell(w)+1,
$$

where $T_{i}=T_{s_{i}}$.

The functor $T_{w}$ admits a right adjoint functor $G_{w}$ in the category $\mathcal{O}^{\mathfrak{g}}([\mathrm{AS}, \S 4])$ :

$$
G_{w}(M)=\mathcal{H o m}_{U}\left(S_{w}, \phi_{w}^{-1}(M)\right) .
$$

It is straightforward to extend the definition of $T_{w}$ and $G_{w}$ to $w \in \mathcal{W}^{e}$ (A1]).

The following assertion follows in the same manner as Soe2, Theorem 2.1].

Lemma 4.1. Let $M \in \mathcal{O}^{\mathfrak{g}}, w \in \mathcal{W}^{e}$,

(i) Suppose that $M$ is free over $\mathfrak{n}_{w}$. Then $M \cong G_{w} T_{w}(M)$. 
(ii) Suppose that $M$ is cofree over $w\left(\mathfrak{n}_{w}\right)$. Then $M \cong T_{w} G_{w}(M)$.

For $\lambda \in \mathfrak{h}^{*}$, let $M(\lambda)$ be the Verma module of $\mathfrak{g}$ with highest weight $\lambda$. Set

$$
M^{w}(\lambda)=T_{w} M\left(w^{-1} \circ \lambda\right) .
$$

The $\mathfrak{g}$-module $M^{w}(\lambda) \in \mathcal{O}^{\mathfrak{g}}$ is called the twisted Verma module $M^{w}(\lambda)$ with highest weight $\lambda$ and twist $w \in \mathcal{W}^{e}$. Note that by (20) we have

$$
\begin{aligned}
M^{w}(\lambda)_{\mu} & \cong \phi_{w}\left(N_{w}^{*} \otimes_{N_{w}} U\left(\mathfrak{n}_{-}\right)\right)_{\mu-\lambda} \\
& \cong\left(U\left(w\left(\mathfrak{n}_{-}\right) \cap \mathfrak{n}_{+}\right)^{*} \otimes_{\mathbb{C}} U\left(w\left(\mathfrak{n}_{-}\right) \cap \mathfrak{n}_{-}\right)\right)_{\mu-\lambda}
\end{aligned}
$$

as $\mathfrak{h}$-modules. Hence

$$
\operatorname{ch} M^{w}(\lambda)=\operatorname{ch} M(\lambda) .
$$

In particular $M^{w}(\lambda)$ is an object of $\mathcal{O}^{\mathfrak{g}}$.

By Lemma 4.1(i) we have

$$
M(\mu) \cong G_{w} M^{w}(w \circ \mu) .
$$

Hence the functor $T_{w}$ gives the isomorphism

$$
\operatorname{Hom}_{\mathfrak{g}}(M(\lambda), M(\mu)) \stackrel{\sim}{\rightarrow} \operatorname{Hom}_{\mathfrak{g}}\left(M^{w}(w \circ \lambda), M^{w}(w \circ \mu)\right)
$$

for $\lambda, \mu \in \mathfrak{h}^{*}$.

We have [AL, Proposition 6.3]

$$
M^{w}(\lambda) \cong M(\lambda) \quad \text { if }\left\langle\lambda+\rho, \alpha^{\vee}\right\rangle \notin \mathbb{N} \text { for all } \alpha \in \Delta_{+}^{r e} \cap w\left(\Delta_{-}^{r e}\right) .
$$

4.3. Hom spaces between twisted Verma modules. For $\lambda \in \mathfrak{h}^{*}$ let $\Delta(\lambda)$ and $\mathcal{W}(\lambda)$ be its integral root system and integral Weyl group, respectively:

$$
\begin{aligned}
& \Delta(\lambda)=\left\{\alpha \in \Delta^{r e} ;\left\langle\lambda+\rho, \alpha^{\vee}\right\rangle \in \mathbb{Z}\right\}, \\
& \mathcal{W}(\lambda)=\left\langle s_{\alpha} ; \alpha \in \Delta(\lambda)\right\rangle \subset \mathcal{W} .
\end{aligned}
$$

Let $\Delta(\lambda)_{+}=\Delta(\lambda) \cap \Delta_{+}^{r e}$ be the set of positive roots of $\Delta(\lambda), \Pi(\lambda) \subset \Delta(\lambda)_{+}$the set of simple roots of $\Delta(\lambda)$, and $\ell: \mathcal{W}(\lambda) \rightarrow \mathbb{Z}_{\geq 0}$ the length function.

For $y \in \mathcal{W}(\lambda)$ the twisted length function $\ell^{y}$ and the twisted Bruhat ordering $\succeq_{\lambda, y}$ are defined for $\mathcal{W}(\lambda)$. We will use the symbol $w \triangleright_{\lambda, y} w^{\prime}$ to denote a covering in the twisted Bruhat order $\succeq_{\lambda, y}$.

Recall that a weight $\lambda \in \mathfrak{h}^{*}$ is called regular dominant if

$$
\left\langle\lambda+\rho, \alpha^{\vee}\right\rangle \notin\{0,-1,-2, \ldots\}
$$

for all $\alpha \in \Delta_{+}^{r e}$. It is called regular anti-dominant if

$$
\left\langle\lambda+\rho, \alpha^{\vee}\right\rangle \notin\{0,1,2, \ldots\}
$$

for all $\alpha \in \Delta_{+}^{r e}$.

Theorem 4.2. Let $w, w^{\prime}, y \in \mathcal{W}(\lambda)$.

(i) If $\lambda$ is regular dominant, then

$$
\operatorname{dim}_{\mathbb{C}} \operatorname{Hom}_{\mathfrak{g}}\left(M^{y}(w \circ \lambda), M^{y}\left(w^{\prime} \circ \lambda\right)\right)= \begin{cases}1 & \text { if } w \succeq_{\lambda, y} w^{\prime} \\ 0 & \text { otherwise. }\end{cases}
$$

(ii) If $\lambda$ is regular anti-dominant, then

$$
\operatorname{dim}_{\mathbb{C}} \operatorname{Hom}_{\mathfrak{g}}\left(M^{y}(w \circ \lambda), M^{y}\left(w^{\prime} \circ \lambda\right)\right)= \begin{cases}1 & \text { if } w \preceq \lambda, y \\ 0 & \text { otherwise }\end{cases}
$$


Proof. (i) By (23) the assertion follows from (16) and [KT, Proposition 2.5.5 (ii)]. Proof of (ii) is similar.

4.4. Wakimoto modules. Let $\mathfrak{g}, \mathfrak{h}$ be as in $₫ 3.1$, and let us consider the $\mathbb{Z}$-grading of $\mathfrak{g}$ with $\mathfrak{g}_{0}=\mathfrak{h}, \mathfrak{g}_{1}=\bigoplus_{\alpha \in \Pi} \mathfrak{g}_{\alpha}$, where $\mathfrak{g}_{\alpha}$ is the root space of $\mathfrak{g}$ of root $\alpha$. Let $\rho=\stackrel{\circ}{\rho}+h^{\vee} \Lambda_{0} \in \mathfrak{h}^{*}$, where $h^{\vee}$ is the dual Coxeter number of $\stackrel{\circ}{\mathfrak{g}}$. Then $\left\langle\rho, \alpha^{\vee}\right\rangle=1$ for all $\alpha \in \Pi$ and $2 \rho$ define a semi-infinite 1-cochain of $\mathfrak{g}$ Ark2.

Let $L \stackrel{\circ}{\mathfrak{n}}, L \stackrel{\circ}{\mathfrak{n}}_{-}, \mathfrak{a}$ and $\overline{\mathfrak{a}}$ be graded subalgebras of $\mathfrak{g}$ defined by

$$
\begin{aligned}
& L \stackrel{\circ}{\mathfrak{n}}=\stackrel{\circ}{\mathfrak{n}}\left[t, t^{-1}\right], \quad L \stackrel{\circ}{\mathfrak{n}}_{-}=\stackrel{\circ}{\mathfrak{n}}_{-}\left[t, t^{-1}\right], \\
& \mathfrak{a}=L \stackrel{\circ}{\mathfrak{n}} \oplus \stackrel{\circ}{\mathfrak{h}}\left[t^{-1}\right] t^{-1}, \quad \overline{\mathfrak{a}}=L \stackrel{\circ}{\mathfrak{n}_{-}} \oplus \stackrel{\circ}{\mathfrak{h}}[t] \oplus \mathbb{C} K \oplus \mathbb{C} D .
\end{aligned}
$$

Then $0=\left.2 \rho\right|_{L \mathfrak{n}} ^{\circ}=\left.2 \rho\right|_{L \mathfrak{n}_{-}}=\left.2 \rho\right|_{\mathfrak{a}}$ gives semi-infinite 1 -cochains of $L \stackrel{\circ}{\mathfrak{n}}, L \stackrel{\circ}{\mathfrak{n}}-\mathfrak{a}$, and $\left.2 \rho\right|_{\overline{\mathfrak{a}}}$ gives a semi-infinite 1 -cochain of $\overline{\mathfrak{a}}$.

Following Vor2 we define the Wakimoto module $W(\lambda)$ of $\mathfrak{g}$ with highest weight $\lambda \in \mathfrak{h}^{*}$ by

$$
W(\lambda)=\mathrm{S} \text {-ind } \frac{\mathfrak{g}}{\mathfrak{a}} \mathbb{C}_{\lambda},
$$

where $\mathbb{C}_{\lambda}$ is the one-dimensional representation of $\mathfrak{h}$ corresponding to $\lambda$ regarded as a $\overline{\mathfrak{a}}$-module by the natural projection $\overline{\mathfrak{a}} \rightarrow \mathfrak{h}$. By Lemma 2.5 we have

$$
W(\lambda) \cong U S(\mathfrak{a}) \text { as } \mathfrak{a} \text {-modules, }
$$

and hence

$$
\begin{aligned}
& H^{\frac{\infty}{2}+i}(\mathfrak{a}, W(\lambda)) \cong\left\{\begin{array}{ll}
\mathbb{C}_{\lambda} & \text { if } i=0, \\
0 & \text { otherwise }
\end{array} \text { as } \mathfrak{h}\right. \text {-modules, } \\
& \operatorname{ch} W(\lambda)=\operatorname{ch} M(\lambda) .
\end{aligned}
$$

In particular $W(\lambda)$ is an object of $\mathcal{O}^{\mathfrak{g}}$.

Theorem 4.7 below shows that the above definition of Wakimoto module coincides with that of Feigin and Frenkel FF2, Fre2.

4.5. Wakimoto modules as inductive limits of twisted Verma modules. Let $y, w, u \in \mathcal{W}$ such that $w=y u$ and $\ell(w)=\ell(y)+\ell(u)$. Then $T_{w}=T_{y} T_{u}$ and $S_{w} \cong S_{y} \otimes_{U} \phi_{y}\left(S_{u}\right)$. Let

$$
j_{w, y}: S_{y} \longrightarrow S_{w}
$$

be the homomorphism of left $U$-modules which maps $s \in S_{y}$ to $s \otimes \mathbf{1}_{u}^{*} \in S_{y} \otimes_{U} \phi_{y}\left(S_{u}\right)$ $=S_{w}$. Define $\nu_{w, y}^{\lambda} \in \operatorname{Hom}_{\mathfrak{g}}\left(M^{y}(\lambda), M^{w}(\lambda)\right)$ by

$$
\nu_{w, y}^{\lambda}\left(s \otimes v_{y^{-1} \circ \lambda}\right)=j_{w, y}(s) \otimes v_{w^{-1} \circ \lambda} \quad \text { for } s \in S_{y},
$$

where $v_{\mu}$ denotes the highest weight vector of $M(\mu)$ for $\mu \in \mathfrak{h}^{*}$. Then

$$
\operatorname{Hom}\left(M^{y}(\lambda), M^{w}(\lambda)\right)=\mathbb{C} \nu_{w, y}^{\lambda}
$$

by (23). We have

$$
\nu_{w_{3}, w_{2}}^{\lambda} \circ \nu_{w_{2}, w_{1}}^{\lambda}=\nu_{w_{3}, w_{1}}^{\lambda}
$$

if $w_{3}=w_{2} u_{2}, w_{2}=w_{1} u_{1}$ with $\ell\left(w_{1}\right)=\ell\left(w_{2}\right)+\ell\left(u_{2}\right), \ell\left(w_{2}\right)=\ell\left(w_{1}\right)+\ell\left(u_{1}\right)$. 
Let $\left\{\gamma_{1}, \gamma_{2}, \ldots\right\}$ be a sequence in $\stackrel{\circ}{P_{+}^{\vee}}$ such that $\gamma_{i}-\gamma_{i-1} \in \stackrel{\circ}{P_{+}^{\vee}}$ and $\lim _{n \rightarrow \infty} \alpha\left(\gamma_{n}\right)=$ $\infty$ for all $\alpha \in \stackrel{\circ}{\Delta}_{+}$. Then $t_{-\gamma_{i+1}}=t_{-\gamma_{i}} t_{-\left(\gamma_{i+1}-\gamma_{i}\right)}$ with $\ell\left(t_{-\gamma_{i+1}}\right)=\ell\left(t_{-\gamma_{i}}\right)+$ $\ell\left(t_{-\left(\gamma_{i+1}-\gamma_{i}\right)}\right)$ for all $i$. It follows that $\left\{M^{-\gamma_{n}}(\lambda): \nu_{-\gamma_{m},-\gamma_{n}}^{\lambda}\right\}$ forms an inductive system of $\mathfrak{g}$-modules.

Proposition 4.3 ([Ark1, Lemma 6.1.7]). There is an isomorphism of $\mathfrak{g}$-modules

$$
W(\lambda) \cong \underset{n}{\lim _{n}} M^{-\gamma_{n}}(\lambda) \text {. }
$$

Proof. For the reader's convenience we shall give a proof of Proposition 4.3 here. Set $W(\lambda)^{\prime}=\lim _{\vec{n}} M^{-\gamma_{n}}(\lambda)$. First note that

$$
\begin{aligned}
& t_{-\gamma_{i}}\left(\mathfrak{n}_{-\gamma_{i}}\right)=t_{-\gamma_{i}}\left(\mathfrak{n}_{-}\right) \cap \mathfrak{n}_{+}=\operatorname{span}_{\mathbb{C}}\left\{x_{\alpha} t^{n} ; \alpha \in \Delta_{+}, 0 \leq n<\alpha\left(\gamma_{i}\right)\right\} \text {, }
\end{aligned}
$$

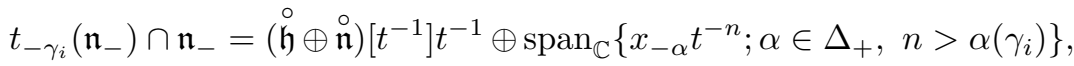

where $x_{\alpha}$ is a root vector of $\stackrel{\circ}{\mathfrak{g}}$ of root $\alpha$. Thus we have $t_{-\gamma_{1}}\left(\mathfrak{n}_{-\gamma_{1}}\right) \subset t_{-\gamma_{2}}\left(\mathfrak{n}_{-\gamma_{2}}\right) \subset$ $\cdots \subset \mathfrak{a}_{+}$and $\mathfrak{a}_{+}=\bigcup_{i \geq 1} t_{-\gamma_{i}}\left(\mathfrak{n}_{-\gamma_{i}}\right)$. The map $j_{-\gamma_{i},-\gamma_{j}}: S_{-\gamma_{i}} \rightarrow S_{-\gamma_{j}}$ restricts to the embedding $j_{-\gamma_{i},-\gamma_{j}}: N_{-\gamma_{i}}^{*} \hookrightarrow N_{-\gamma_{j}}^{*}$ for $i<j$, and we have

$$
U\left(\mathfrak{a}_{+}\right)^{*} \cong \lim _{\vec{i}} \phi_{-\gamma_{i}}\left(N_{-\gamma_{i}}^{*}\right)
$$

as left $\mathfrak{a}_{+}$-modules. Let $j_{-\gamma_{i}}: \phi_{-\gamma_{i}}\left(N_{-\gamma_{i}}^{*}\right) \hookrightarrow U\left(\mathfrak{a}_{+}\right)^{*}$ be the embedding of left $\phi_{-\gamma_{i}}\left(N_{-\gamma_{i}}\right)$-modules under the above identification.

Since $t_{-\gamma_{i}}\left(\mathfrak{n}_{-\gamma_{i}}\right)=\operatorname{span}_{\mathbb{C}}\left\{x_{\alpha} t^{-n} ; \alpha \in \Delta_{+}, 0<n \leq \alpha\left(\gamma_{i}\right)\right\} \subset \mathfrak{a}$,

$$
W(\lambda) \cong T_{-\gamma_{i}} G_{-\gamma_{i}}(W(\lambda))
$$

by Lemma 4.1(ii). Hence

$$
\operatorname{Hom}_{\mathfrak{g}}\left(M^{-\gamma_{i}}(\lambda), W(\lambda)\right) \cong \operatorname{Hom}_{\mathfrak{g}}\left(M\left(t_{\gamma_{i}} \circ \lambda\right), G_{-\gamma_{i}}(W(\lambda))\right) .
$$

As $\operatorname{ch} G_{-\gamma_{i}}(W(\lambda))=\operatorname{ch} M\left(t_{\gamma_{i}} \circ \lambda\right)$, there exists a unique $\mathfrak{g}$-module homomorphism $\psi_{i}: M\left(t_{\gamma_{i}} \circ \lambda\right) \rightarrow G_{-\gamma_{i}}(M)$ which sends $v_{t_{\gamma_{i}} \circ \lambda}$ to $w_{i}$, a vector of $G_{-\gamma_{i}}(W(\lambda))$ of weight $t_{\gamma_{i}} \circ \lambda$. Up to a non-zero constant multiplication, $w_{i}$ is equal to the element of $G_{-\gamma_{i}}(W(\lambda))=\mathcal{H o m}_{N_{-\gamma_{i}}}\left(N_{-\gamma_{i}}^{*}, \phi_{-\gamma_{i}}^{-1}(W(\lambda))\right)$ which sends $f \in N_{t_{-\gamma_{i}}}^{*}$ to $j_{-\gamma_{i}}(f) \otimes 1_{\lambda} \in U S(\mathfrak{a}) \otimes \mathbb{C}_{\lambda}=W(\lambda)$. The corresponding homomorphism $T_{-\gamma_{i}}\left(\psi_{i}\right)$ : $M^{-\gamma_{i}}(\lambda) \rightarrow W(\lambda)$ is given by

$$
T_{-\gamma_{i}}\left(\psi_{i}\right)\left(f \otimes v_{t_{\gamma_{i}} \circ \lambda}\right)=j_{-\gamma_{i}}(f) \otimes 1_{\lambda} \quad \text { for } f \in N_{-\gamma_{i}}^{*} .
$$

It follows that $T_{-\gamma_{i}}\left(\psi_{j}\right) \circ \nu_{\gamma_{j}, \gamma_{i}}^{\lambda}=T_{-\gamma_{i}}\left(\psi_{i}\right)$ for $i<j$, and the sequence $\left\{T_{-\gamma_{i}}\left(\psi_{j}\right)\right\}$ yields a $\mathfrak{g}$-module homomorphism

$$
\Phi: W(\lambda)^{\prime}=\underset{i}{\lim } M^{-\gamma_{i}}(\lambda) \longrightarrow W(\lambda) .
$$

Fix $\mu \in \mathfrak{h}^{*}$. Since $W(\lambda) \cong U S(\mathfrak{a})$ as an $\mathfrak{a}$-module, it follows from (22) that $T_{-\gamma_{i}}$ restricts to the isomorphism $M^{-\gamma_{i}}(\lambda)_{\mu} \stackrel{\sim}{\rightarrow} W(\lambda)_{\mu}$ for a sufficiently large $i$. This completes the proof. 


\subsection{Endmorphisms of Wakimoto modules.}

Proposition 4.4. Let $\alpha \in \stackrel{\circ}{P_{+}^{\vee}}, \lambda \in \mathfrak{h}^{*}$.

(i) $T_{-\alpha} W(\lambda) \cong W\left(t_{-\alpha} \circ \lambda\right)$.

(ii) $G_{-\alpha} W(\lambda) \cong W\left(t_{\alpha} \circ \lambda\right)$.

Proof. (i) Let $\left\{\gamma_{1}, \gamma_{2}, \ldots\right\}$ be a sequence in $\stackrel{\circ}{P_{+}^{\vee}}$ such that $\gamma_{i}-\gamma_{i-1} \in \stackrel{\circ}{P_{+}^{\vee}}$ and $\lim _{n \rightarrow \infty} \beta\left(\gamma_{n}\right)=\infty$ for all $\beta \in \stackrel{\circ}{\Delta}_{+}$. Set $\gamma_{i}^{\prime}=\gamma_{i}+\alpha$. Then the sequence $\left\{\gamma_{1}^{\prime}, \gamma_{2}^{\prime}, \ldots\right\}$ satisfies the same property. Hence by Proposition 4.3 and the fact that a homology functor commutes with inductive limits we have $T_{-\alpha} W(\lambda) \cong T_{-\alpha}\left(\lim _{\longrightarrow} M^{-\gamma_{i}}(\lambda)\right)=$ $\underset{\longrightarrow}{\lim } T_{-\alpha} M^{-\gamma_{i}}(\lambda)=\lim _{\longrightarrow} T_{-\alpha} T_{-\gamma_{i}} M\left(t_{\gamma_{i}} \circ \lambda\right)=\lim _{\longrightarrow} T_{-\gamma_{i}^{\prime}} M\left(t_{\gamma_{i}} \circ \lambda\right)=\lim _{\longrightarrow} M^{-\gamma_{i}^{\prime}}\left(t_{\alpha} \circ\right.$ $\overrightarrow{\lambda)} \cong W\left(t_{\alpha} \circ \lambda\right)$. (ii) Since $\mathfrak{n}_{t_{-\alpha}} \subset \mathfrak{a}_{-}, W(\lambda)$ is free over $\mathfrak{n}_{t_{-\alpha}}$. Hence $W\left(t_{\alpha} \circ \lambda\right)=$ $G_{-\alpha} T_{-\alpha} W\left(t_{\alpha} \circ \lambda\right) \cong G_{-\alpha} W(\lambda)$ by Lemma 4.1 and (i).

Corollary 4.5. Let $\alpha \in \stackrel{\circ}{P_{+}^{\vee}}$. The functor $G_{-\alpha}$ gives the isomorphism

$$
\operatorname{Hom}_{\mathfrak{g}}(W(\lambda), W(\mu)) \cong \operatorname{Hom}_{\mathfrak{g}}\left(W\left(t_{\alpha} \circ \lambda\right), W\left(t_{\alpha} \circ \mu\right)\right)
$$

for $\lambda, \mu \in \mathfrak{h}^{*}$.

Proposition 4.6. For $\lambda \in \mathfrak{h}^{*}$ we have $\operatorname{End}_{\mathfrak{g}}(W(\lambda))=\mathbb{C}$.

Proof. Let $\left\{\gamma_{1}, \gamma_{2}, \ldots,\right\}$ be in Subsection 4.5. Then

$$
\begin{aligned}
& \operatorname{End}_{\mathfrak{g}}(W(\lambda))=\operatorname{Hom}_{\mathfrak{g}}\left(\underset{\vec{i}}{\lim } M^{-\gamma_{i}}(\lambda), W(\lambda)\right) \quad \text { (by Proposition 4.3) } \\
& =\lim _{\leftarrow} \operatorname{Hom}_{\mathfrak{g}}\left(M^{-\gamma_{i}}(\lambda), W(\lambda)\right) \cong \lim _{\leftarrow} \operatorname{Hom}_{\mathfrak{g}}\left(M\left(t_{\gamma_{i}} \circ \lambda\right), G_{-\gamma_{i}} W(\lambda)\right) \\
& \cong \lim _{\leftarrow} \operatorname{Hom}_{\mathfrak{g}}\left(M\left(t_{\gamma_{i}} \circ \lambda\right), W\left(t_{\gamma_{i}} \circ \lambda\right)\right) \quad \text { (by Proposition 4.4). }
\end{aligned}
$$

As we have seen in the proof of Proposition 4.3, the space $\operatorname{Hom}_{\mathfrak{g}}\left(M\left(t_{\gamma_{i}} \circ \lambda\right)\right.$, $\left.W\left(t_{\gamma_{i}} \circ \lambda\right)\right)$ is one-dimensional and $\nu_{-\gamma_{m}, \gamma_{n}}^{\lambda}$ induces the isomorphism

$$
\operatorname{Hom}_{\mathfrak{g}}\left(M^{-\gamma_{m}}(\lambda), W(\lambda)\right) \stackrel{\sim}{\rightarrow} \operatorname{Hom}_{\mathfrak{g}}\left(M^{-\gamma_{n}}(\lambda), W(\lambda)\right) .
$$

This completes the proof.

4.7. Uniqueness of Wakimoto modules. A finite filtration $0=M_{0} \subset M_{1} \subset$ $M_{2} \subset M_{r}=M$ of a $\mathfrak{g}$-module $M$ is called a Wakimoto flag if each successive quotient $M_{i} / M_{i-1}$ is isomorphic to $W\left(\lambda_{i}\right)$ for some $\lambda_{i}$.

Theorem 4.7. Suppose that $k$ is non-critical, that is, $k \neq-h^{\vee}$. For an object $M$ of $\mathcal{O}_{k}^{\mathfrak{g}}$ the following conditions are equivalent.

(i) $M$ admits a Wakimoto flag.

(ii) $H^{\frac{\infty}{2}+i}(\mathfrak{a}, M)=0$ for $i \neq 0$ and $H^{\frac{\infty}{2}+0}(\mathfrak{a}, M)$ is finite-dimensional.

If this is the case the multiplicity $(M: W(\lambda))$ of $W(\lambda)$ in a Wakimoto flag of $M$ equals to $\operatorname{dim} H^{\frac{\infty}{2}+0}(\mathfrak{a}, M)_{\lambda}$. In particular if

$$
H^{\frac{\infty}{2}+i}(\mathfrak{a}, M) \cong \begin{cases}\mathbb{C}_{\lambda} & \text { if } i=0 \\ 0 & \text { otherwise }\end{cases}
$$

as $\mathfrak{h}$-modules, $M$ is isomorphic to $W(\lambda)$.

The proof of Theorem 4.7 will be given in Subsection 4.8 . 
We put on record some consequences of Theorem 4.7

Proposition 4.8. A tilting module in $\mathcal{O}^{\mathfrak{g}}$ at a non-critical level admits a Wakimoto flag.

Proof. By definition a tilting module $M$ admits both a Verma flag and a dual Verma flag. It follows that $M$ is free over $\mathfrak{n}_{-}$and cofree over $\mathfrak{n}_{+}$. In particular $M$ is free over $\mathfrak{n}\left[t^{-1}\right] t^{-1}$ and cofree over $\mathfrak{\mathfrak { n }}[t]$. Hence by [Vor1, Theorem 2.1], we have $H^{\frac{\infty}{2}+i}(\mathfrak{a}, M)=0$ for $i \neq 0$. The fact that $H^{\frac{\infty}{2}+0}(\mathfrak{a}, M)$ is finite-dimensional follows from the Euler-Poincaré principle.

Proposition 4.9. Suppose that $\langle\lambda+\rho, K\rangle \notin \mathbb{Q}_{\geq 0}$. Then $W\left(t_{\alpha} \circ \lambda\right) \cong M\left(t_{\alpha} \circ \lambda\right)$ for a sufficiently large $\alpha \in \stackrel{\circ}{P_{+}^{\vee}}$.

Proof. Let $\alpha$ be sufficiently large. By the hypothesis $\left\langle t_{\alpha}(\lambda+\rho), \beta^{\vee}\right\rangle \notin \mathbb{N}$ for all $\beta \in \Delta_{+}^{r e}$ such that $\bar{\beta} \in \stackrel{\circ}{\Delta}_{+}$. It follows from [A1, Theorem 3.1] that $M\left(t_{\alpha} \circ \lambda\right)$ is cofree over $\stackrel{\circ}{\mathfrak{n}}[t]=\mathfrak{a}_{+}$. Because $M\left(t_{\alpha} \circ \lambda\right)$ is obviously free over $\mathfrak{a}_{-}$we have

$$
H^{\frac{\infty}{2}+i}\left(\mathfrak{a}, M\left(t_{\alpha} \circ \lambda\right)\right) \cong \begin{cases}\mathbb{C}_{t_{\alpha} \circ \lambda} & \text { for } i=0, \\ 0 & \text { otherwise. }\end{cases}
$$

The following assertion follows from Proposition 4.9 and Corollary 4.5

Proposition 4.10. Let $\lambda, \mu \in \mathfrak{h}^{*}$ be of level $k$, and suppose that $k+h^{\vee} \notin \mathbb{Q} \geq 0$. Then

$$
\operatorname{Hom}_{\mathfrak{g}}(W(\lambda), W(\mu)) \cong \operatorname{Hom}_{\mathfrak{g}}\left(M\left(t_{\alpha} \circ \lambda\right), M\left(t_{\alpha} \circ \mu\right)\right)
$$

for a sufficiently large $\alpha \in \stackrel{\circ}{P}_{+}^{\vee}$. In particular if $\lambda \in \mathfrak{h}^{*}$ is integral, regular antidominant, then

$$
\operatorname{dim}_{\mathbb{C}} \operatorname{Hom}_{\mathfrak{g}}(W(w \circ \lambda), W(y \circ \lambda))= \begin{cases}1 & \text { if } w \preceq \frac{\infty}{2} y \\ 0 & \text { else },\end{cases}
$$

for $w, y \in \mathcal{W}$.

Conjecture 4.11. Let $\lambda \in \mathfrak{h}^{*}$ be integral, regular dominant. Then

$$
\operatorname{dim}_{\mathbb{C}} \operatorname{Hom}_{\mathfrak{g}}(W(w \circ \lambda), W(y \circ \lambda))= \begin{cases}1 & \text { if } w \succeq \frac{\infty}{2} y, \\ 0 & \text { else },\end{cases}
$$

for $w, y \in \mathcal{W}$.

In Theorem 6.11 below we prove Conjecture 4.11 in the case that $w \triangleright \frac{\infty}{2} y$ (in a slightly more general setting).

\subsection{Proof of Theorem 4.7, Let}

$$
\mathcal{H}=\stackrel{\circ}{\mathfrak{h}}\left[t, t^{-1}\right] \oplus \mathbb{C} K \subset \mathfrak{g},
$$

the Heisenberg subalgebra. Denote by $\pi_{\lambda}$ the irreducible representation of $\mathcal{H}$ with highest weight $\lambda$. We have $\pi_{\lambda} \cong U\left(\mathfrak{h}\left[t^{-1}\right] t^{-1}\right)$ as a module over $\stackrel{\circ}{\mathfrak{h}}\left[t^{-1}\right] t^{-1} \subset \mathcal{H}$ provided that $\lambda(K) \neq 0$.

For $M \in \mathcal{O}_{k}^{\mathfrak{g}}$ one knows that $H^{\frac{\infty}{2}+} \bullet(L \stackrel{\circ}{\mathfrak{n}}, M)$ is naturally an $\mathcal{H}$-module of level $k+h^{\vee}($ FF2 $)$. 
Lemma 4.12. Let $M$ be an object of $\mathcal{O}_{k}^{\mathfrak{g}}$ with $k \neq-h^{\vee}$. Then the following conditions are equivalent:

(i) $H^{\frac{\infty}{2}+i}(\mathfrak{a}, M)=0$ for $i \neq 0$;

(ii) $H^{\frac{\infty}{2}+i}(L \mathfrak{\circ}, M)=0$ for $i \neq 0$.

Proof. The assumption that $k \neq-h^{\vee}$ implies that $H^{\frac{\infty}{2}+\bullet}(L \stackrel{\circ}{\mathfrak{n}}, M)$ is semi-simple as an $\mathcal{H}$-module and is a direct sum of $\pi_{\mu} \mathrm{s}$. Consider the Hochschild-Serre spectral sequence for the ideal $L \stackrel{\circ}{\mathfrak{n}} \subset \mathfrak{a}$ to compute $H^{\frac{\infty}{2}+\bullet}(\mathfrak{a}, M)$. By definition, we have

$$
E_{2}^{p, q}= \begin{cases}H_{-p}\left(\stackrel{\circ}{\mathfrak{h}}\left[t^{-1}\right] t^{-1}, H^{\frac{\infty}{2}+q}(L \stackrel{\circ}{\mathfrak{n}}, M)\right) & \text { for } p \leq 0 \\ 0 & \text { for } p>0 .\end{cases}
$$

By the above mentioned fact $H^{\frac{\infty}{2}+q}(L \stackrel{\circ}{\mathfrak{n}}, M)$ is free over $U\left(\stackrel{\circ}{\mathfrak{h}}\left[t^{-1}\right] t^{-1}\right)$. Hence

$$
E_{2}^{p, q}= \begin{cases}\left.H^{\frac{\infty}{2}+q}(L \mathfrak{\circ}, M) / \stackrel{\circ}{\mathfrak{h}}\left[t^{-1}\right] t^{-1}\left(H^{\frac{\infty}{2}+q}(L \mathfrak{\circ}, M)\right)\right) & \text { for } p=0, \\ 0 & \text { for } p \neq 0 .\end{cases}
$$

Therefore the spectral sequence collapses at $E_{2}=E_{\infty}$, and $H^{\frac{\infty}{2}+i}(\mathfrak{a}, M)=0$ for $i \neq 0$ if and only if $H^{\frac{\infty}{2}+i}(L \mathfrak{n}, M)=0$ for $i \neq 0$. This completes the proof.

Proposition 4.13. Let $M$ be an object of $\mathcal{O}_{k}$ at a non-critical level $k$ such that $H^{\frac{\infty}{2}+i}(\mathfrak{a}, M)=0$ for $i \neq 0$. Then

$$
M \cong U S(\mathfrak{a}) \otimes_{\mathbb{C}} H^{\frac{\infty}{2}+0}(\mathfrak{a}, M)
$$

as $\mathfrak{a}$-modules and $\mathfrak{h}$-modules, where $\mathfrak{a}$ acts only on the first factor $U S(\mathfrak{a})$ and $\mathfrak{h}$ acts as $h(s \otimes m)=\operatorname{ad}(h)(s) \otimes m+s \otimes h m$.

Proof. By Proposition 2.3 it suffices to show that S-ind $\mathfrak{a}_{\mathfrak{a}}^{\mathfrak{a}} M \cong U S(\mathfrak{a}) \otimes_{\mathbb{C}} H^{\frac{\infty}{2}+0}(\mathfrak{a}, M)$. As in the proof of Lemma 4.12, we shall consider the Hochschild-Serre spectral sequence for the ideal $\stackrel{\circ \mathfrak{n}}{\subset} \mathfrak{a}$ to compute $H^{\frac{\infty}{2}+\bullet}(\mathfrak{a}, U S(\mathfrak{a}) \otimes M)$. By definition we have

$$
\begin{aligned}
& E_{1}^{\bullet, q}=H^{\frac{\infty}{2}+q}\left(\stackrel{\circ}{\mathfrak{n}}, U S(\mathfrak{a}) \otimes_{\mathbb{C}} M\right) \otimes_{\mathbb{C}} \bigwedge^{\bullet}\left(\stackrel{\circ}{\mathfrak{h}}\left[t^{-1}\right] t^{-1}\right), \\
& E_{2}^{p, q}=H_{-p}\left(\stackrel{\circ}{\mathfrak{h}}\left[t^{-1}\right] t^{-1}, H^{\frac{\infty}{2}+q}\left(L \stackrel{\circ}{\operatorname{n}}, U S(\mathfrak{a}) \otimes_{\mathbb{C}} M\right)\right) .
\end{aligned}
$$

To compute the $E_{1}$-term set

$$
F^{p} U S(\mathfrak{a})=\bigoplus_{\substack{0 \\\left\langle\mu, \rho^{\vee}\right\rangle \geq p}} U S(\mathfrak{a})_{\mu}
$$

where $U S(\mathfrak{a})$ is considered as an $\mathfrak{h}$-module by the adjoint action. Then

$$
\begin{aligned}
& U S(\mathfrak{a})=F^{0} U S(\mathfrak{a}) \supset F^{1} U S(\mathfrak{a}) \supset \ldots, \quad \bigcap F^{p} U S(\mathfrak{a})=0, \\
& F^{p} U S(\mathfrak{a}) \cdot L \stackrel{\circ}{\mathfrak{n}} \subset F^{p+1} U S(\mathfrak{a}) .
\end{aligned}
$$

Define the filtration $F^{\bullet}\left(U S(\mathfrak{a}) \otimes_{\mathbb{C}} M \otimes_{\mathbb{C}} \bigwedge^{\frac{\infty}{2}+\bullet}(L \mathfrak{i})\right)$ by setting

$$
F^{p}\left(U S(\mathfrak{a}) \otimes_{\mathbb{C}} M \otimes_{\mathbb{C}} \bigwedge^{\frac{\infty}{2}+\bullet}(L \stackrel{\circ}{\mathfrak{n}})\right)=F^{p} U S(\mathfrak{a}) \otimes_{\mathbb{C}} M \otimes_{\mathbb{C}} \bigwedge^{\frac{\infty}{2}+\bullet}(L \stackrel{\circ}{\mathfrak{n}}) .
$$

This defines a decreasing, weightwise regular filtration of the complex. Consider the associated spectral sequence $E_{r}^{\prime} \Rightarrow H^{\frac{\infty}{2}+\bullet}\left(L \mathfrak{\circ}, U S(\mathfrak{a}) \otimes_{\mathbb{C}} M\right)$. Because the associated 
graded space gr $U S(\mathfrak{a})$ with respect to this filtration is a trivial $L \mathfrak{n}$-module the $E_{1^{-}}$ term of the spectral sequence $E_{r}^{\prime}$ is isomorphic to $U S(\mathfrak{a}) \otimes_{\mathbb{C}} H^{\frac{\infty}{2}}+\bullet(L \mathfrak{n}, M)$. Hence by the hypothesis and Lemma 4.12 the spectral sequence $E_{r}^{\prime}$ collapses at $E_{1}^{\prime}=E_{\infty}^{\prime}$ and we obtain the isomorphism of $\mathfrak{h}$-modules

$$
H^{\frac{\infty}{2}+i}\left(L \stackrel{\circ}{\mathfrak{n}}, U S(\mathfrak{a}) \otimes_{\mathbb{C}} M\right) \cong \begin{cases}U S(\mathfrak{a}) \otimes_{\mathbb{C}} H^{\frac{\infty}{2}+0}(L \mathfrak{n}, M) & \text { for } i=0, \\ 0 & \text { for } i \neq 0 .\end{cases}
$$

This is also an isomorphism of $\mathfrak{a}$-modules since $U S(\mathfrak{a}) \cong \operatorname{gr} U S(\mathfrak{a})$ as left $\mathfrak{a}$-modules, where $x_{\alpha} t^{n} \in \mathfrak{a}$ is considered as an operator on $\operatorname{gr} U S(\mathfrak{a})=\bigoplus_{p} F^{p} U S(\mathfrak{a}) / F^{p+1} U S(\mathfrak{a})$ which maps $F^{p} U S(\mathfrak{a}) / F^{p+1} U S(\mathfrak{a})$ to $F^{p+\alpha\left(\stackrel{\circ}{\rho}^{\vee}\right)} U S(\mathfrak{a}) / F^{p+\alpha\left(\stackrel{\circ}{\rho}^{\vee}\right)+1} U S(\mathfrak{a})$. We have computed the $E_{1}$-term (30):

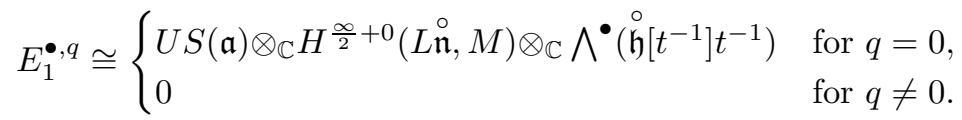

It follows that

$$
E_{2}^{p, q} \cong \begin{cases}U S(\mathfrak{a}) \otimes_{\mathbb{C}} H^{\frac{\infty}{2}+0}(\mathfrak{a}, M) & \text { for } p=q=0, \\ 0 & \text { otherwise, }\end{cases}
$$

as $\mathfrak{h}$-modules and $\mathfrak{a}$-modules, see the proof of Lemma 4.12. The spectral sequence collapses at $E_{2}=E_{\infty}$ and we obtain the required isomorphism.

Set

$$
Q_{\frac{\infty}{2},+}=\sum_{\substack{\alpha \in \Delta^{\mathrm{re}} \\ \bar{\alpha} \in \dot{\Delta}_{-}}} \mathbb{Z}_{\geq 0} \alpha+\mathbb{Z}_{\geq 0} \delta \subset \mathfrak{h}^{*},
$$

and define the partial ordering $\leq_{\frac{\infty}{2}}$ on $\mathfrak{h}^{*}$ by $\mu \leq_{\frac{\infty}{2}} \lambda \Longleftrightarrow \lambda-\mu \in Q_{\frac{\infty}{2},+}$. Note that $\mu \leq_{\frac{\infty}{2}} \lambda$ if and only if $t_{\alpha} \circ \mu \leq t_{\alpha} \circ \lambda$ for a sufficiently large $\alpha \in \stackrel{\circ}{Q^{\vee}}$.

Theorem 4.7. Since The direction (i) $\Rightarrow$ (ii) in Theorem 4.7 is obvious by (26), we shall prove that (ii) implies (i). Let $\left\{\lambda_{1}, \ldots, \lambda_{r}\right\}$ be the set of weights of $H^{\frac{\infty}{2}+0}(\mathfrak{a}, M)$ with multiplicities counted, so that

$$
M \cong \bigoplus_{i=1}^{r} U S(\mathfrak{a}) \otimes_{\mathbb{C}} \mathbb{C}_{\lambda_{i}}
$$

as $\mathfrak{a}$-modules and $\mathfrak{h}$-modules by Proposition 4.13. We may assume that if $\lambda_{i} \leq \frac{\infty}{2} \lambda_{j}$, then $j<i$.

Set $\lambda=\lambda_{1}$. We shall show that there is a $\mathfrak{g}$-module embedding $W(\lambda) \hookrightarrow M$. Let $\left\{\gamma_{1}, \gamma_{2}, \ldots\right\}$ be a sequence in $\stackrel{\circ}{P_{+}^{\vee}}$ such that $\gamma_{i}-\gamma_{i-1} \in \stackrel{\circ}{P_{+}^{\vee}}$ and $\lim _{n \rightarrow \infty} \alpha\left(\gamma_{n}\right)=\infty$ for

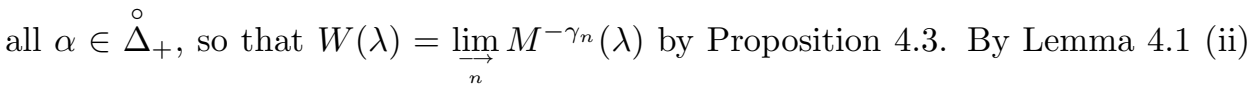
we have $M \cong T_{-\gamma_{i}} G_{-\gamma_{i}}(M)$, and hence,

$$
\operatorname{Hom}_{\mathfrak{g}}\left(M^{-\gamma_{i}}(\lambda), M\right) \cong \operatorname{Hom}_{\mathfrak{g}}\left(M\left(t_{\gamma_{i}} \circ \lambda\right), G_{-\gamma_{i}}(M)\right) .
$$

By (34), $\operatorname{ch} G_{-\gamma_{i}}(M)=\sum_{i=1}^{r} \operatorname{ch} M\left(t_{\gamma_{i}} \circ \lambda\right)$. Let $i$ be sufficiently large so that $t_{\gamma_{i}} \circ \lambda$ is maximal in $G_{-\gamma_{i}}(M)$. Denote by $\Phi_{i}$ the $\mathfrak{g}$-module homomorphism $\psi_{i}$ : $M\left(t_{\gamma_{i}} \circ \lambda\right) \rightarrow G_{-\gamma_{i}}(M)$ which sends $v_{t_{\gamma_{i}} \circ \lambda}$ to a vector of $G_{-\gamma_{i}}(M)$ of weight $t_{\gamma_{i}} \circ \lambda$. 
As in the proof of Proposition $4.3\left\{T_{-\gamma_{i}}\left(\psi_{i}\right): M^{-\gamma_{i}}(\lambda) \mapsto M\right\}$ yield an injective $\mathfrak{g}$-module homomorphism

$$
\Phi: W(\lambda)=\lim _{i} M^{-\gamma_{i}}(\lambda) \hookrightarrow M .
$$

The map $\Phi$ induces the homomorphism $H^{\frac{\infty}{2}+0}(\mathfrak{a}, W(\lambda))=\mathbb{C}_{\lambda} \rightarrow H^{\frac{\infty}{2}+0}(\mathfrak{a}, M)$ which is certainly injective. It follows from the long exact sequence associated with the exact sequence $0 \rightarrow W(\lambda) \stackrel{\Phi}{\rightarrow} M \rightarrow M / W(\lambda) \rightarrow 0$ we obtain that $H^{\frac{\infty}{2}+i}(\mathfrak{a}, M / W(\lambda))=0$ for $i \neq 0$ and $\operatorname{dim} H^{\frac{\infty}{2}+0}(\mathfrak{a}, M / W(\lambda))=\operatorname{dim} H^{\frac{\infty}{2}+0}(\mathfrak{a}, M)-1$. Theorem 4.7 follows by the induction on $\operatorname{dim} H^{\frac{\infty}{2}+0}(\mathfrak{a}, M)$.

4.9. Twisted Wakimoto modules. For $w \in \stackrel{\circ}{\mathcal{W}}$ we have the decomposition $\mathfrak{g}=$ $w(\mathfrak{a}) \oplus w(\overline{\mathfrak{a}})$, and $2 \rho$ defines a semi-infinite 1-cochain of the graded subalgebra $w(\overline{\mathfrak{a}})$. Hence we can define the twisted Wakimoto module $W^{w}(\lambda)$ with highest weight $\lambda$ and twist $w \in \stackrel{\circ}{\mathcal{W}}$ by

$$
W^{w}(\lambda)=\operatorname{S-ind}_{w(\overline{\mathfrak{a}})}^{\mathfrak{g}} \mathbb{C}_{\lambda},
$$

where $\mathbb{C}_{\lambda}$ is the one-dimensional representation of $\mathfrak{h}$ corresponding to $\lambda$ regarded as a $\overline{\mathfrak{a}}$-module by the projection $\overline{\mathfrak{a}} \rightarrow \mathfrak{h}$. We have

$$
\begin{aligned}
& W^{w}(\lambda) \cong U S(w(\mathfrak{a})) \text { as } w(\mathfrak{a}) \text {-modules and } \operatorname{ch} W^{w}(\lambda)=\operatorname{ch} M(\lambda), \\
& H^{\frac{\infty}{2}+i}\left(w(\mathfrak{a}), W^{w}(\lambda)\right) \cong\left\{\begin{array}{ll}
\mathbb{C}_{\lambda} & \text { for } i=0, \\
0 & \text { otherwise, }
\end{array} \text { as } \mathfrak{h}\right. \text {-modules. }
\end{aligned}
$$

Let $\left\{\gamma_{1}, \gamma_{2}, \ldots\right\}$ be a sequence in $\stackrel{\circ}{P_{+}^{\vee}}$ such that $\gamma_{i}-\gamma_{i-1} \in \stackrel{\circ}{P_{+}^{\vee}}$ and $\lim _{n \rightarrow \infty} \alpha\left(\gamma_{n}\right)=$ $\infty$ for all $\alpha \in \stackrel{\circ}{\Delta}_{+}$. The following assertion can be proved in the same manner as Proposition 4.3

Proposition 4.14. Let $\lambda \in \mathfrak{h}^{*}, w \in \stackrel{\circ}{\mathcal{W}}$. There is an isomorphism of $\mathfrak{g}$-modules

$$
W^{w}(\lambda) \cong \underset{n}{\lim } M^{-w\left(\gamma_{n}\right)}(\lambda) .
$$

The following assertion can be proved in the same manner as Theorem 4.7

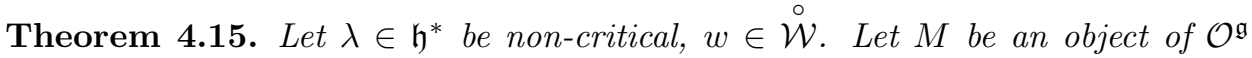
such that

$$
H^{\frac{\infty}{2}+i}(w(\mathfrak{a}), M) \cong \begin{cases}\mathbb{C}_{\lambda} & \text { if } i=0 \\ 0 & \text { otherwise }\end{cases}
$$

as $\mathfrak{h}$-modules. Then $M$ is isomorphic to $W^{w}(\lambda)$.

\section{Borel-Weil-Bott VANiShing PROPERTy OF TWISTING FUnCTORS}

5.1. Left derived functors of twisting functors. The functor $T_{w}, w \in \mathcal{W}^{e}$, admits the left derived functor $\mathcal{L}_{\bullet} T_{w}$ in the category $\mathcal{O}^{\mathfrak{g}}$ since it is a Lie algebra homology functor:

$$
\mathcal{L}_{i} T_{w}(M)=\phi_{w}\left(H_{i}\left(\mathfrak{g}, S_{w} \otimes_{\mathbb{C}} M\right)\right)
$$


where $\mathfrak{g}$ acts on $N_{w}^{*} \otimes_{\mathbb{C}} M$ by $X(f \otimes m)=-f X \otimes m+f \otimes X m$. Because

$$
\mathcal{L}_{i} T_{w}(M) \cong \phi_{w}\left(H_{i}\left(\mathfrak{n}_{w}, N_{w}^{*} \otimes_{\mathbb{C}} M\right)\right)
$$

as $w\left(\mathfrak{n}_{w}\right)$-modules, we have the following assertion.

Lemma 5.1. Suppose $M \in \mathcal{O}^{\mathfrak{g}}$ is free over $\mathfrak{n}_{w}$. Then $\mathcal{L}_{i} T_{w}(M)=0$ for $i \geq 1$.

Let $\left\{e_{i}, h_{i}, f_{i} ; i \in I\right\}, e_{i} \in \mathfrak{g}_{\alpha_{i}}, f_{i} \in \mathfrak{g}_{-\alpha_{i}}$, be the Chevalley generators of $\mathfrak{g}$. For $i \in I$, let $\mathfrak{s l}_{2}^{(i)}$ denote the copy of $\mathfrak{s l}_{2}$ in $\mathfrak{g}$ spanned by $\left\{e_{i}, h_{i}, f_{i}\right\}$

Proposition 5.2. Let $M \in \mathcal{O}^{\mathfrak{g}}, i \in I$. Denote by $N$ the largest $\mathfrak{s l}_{2}^{(i)}$-integrable submodule of $M$. Then $T_{i}(M) \cong T_{i}(M / N)$, $\operatorname{ch} \mathcal{L}_{1} T_{i}(M) \cong \operatorname{ch} N$ and $\mathcal{L}_{p} T_{i}(M)=0$ for $p \geq 2$.

Proof. Let $T_{i}^{(i)}$ denote the twisting functor for $\mathfrak{s l}_{2}^{(i)}$ corresponding to the reflection $s_{\alpha_{i}}$. Because $T_{i}(M) \cong T_{i}^{(i)}(M)$ as $\mathfrak{s l}_{2}^{(i)}$-modules and $\mathfrak{h}$-modules, we have

$$
\mathcal{L}_{p} T_{i}(M) \cong \mathcal{L}_{p} T_{i}^{(i)}(M) \text { as } \mathfrak{s l}_{2}^{(i)} \text {-modules and } \mathfrak{h} \text {-modules. }
$$

In particular $\mathcal{L}_{p} T_{i}(M)=0$ for $p \geq 2$. It follows that the exact sequence

$$
0 \rightarrow N \rightarrow M \rightarrow M / N \rightarrow 0
$$

yields the long exact sequence

$$
\begin{aligned}
0 \rightarrow \mathcal{L}_{1} T_{i}(N) & \rightarrow \mathcal{L}_{1} T_{i}(M) \rightarrow \mathcal{L}_{1} T_{i}(M / N) \\
& \rightarrow T_{i}(N) \rightarrow T_{i}(M) \rightarrow T_{i}(M / N) \rightarrow 0 .
\end{aligned}
$$

Since $M / N$ is free as $\mathbb{C}\left[f_{i}\right]$-module $\mathcal{L}_{1} T_{i}(M / N)=0$ by Lemma 5.1. Also, $T_{i}(N)=0$ and $\mathcal{L}_{1} T_{i}(N) \cong N$ as $\mathfrak{h}$-modules by [AS, Theorem 6.1] and (36). This completes the proof.

Let $L(\lambda) \in \mathcal{O}^{\mathfrak{g}}$ be the irreducible highest weight representation of $\mathfrak{g}$ with highest weight $\lambda \in \mathfrak{h}^{*}$.

Theorem 5.3 ([AS, Theorem 6.1]). Let $\lambda \in \mathfrak{h}^{*}$ and suppose that $\left\langle\lambda, \alpha_{i}^{\vee}\right\rangle \in \mathbb{Z}_{\geq 0}$ with $i \in I$. Then

$$
\mathcal{L}_{p} T_{i}(L(\lambda)) \cong \begin{cases}L(\lambda) & \text { if } p=1 \\ 0 & \text { if } p \neq 1\end{cases}
$$

Proof. The hypothesis implies that $L(\lambda)$ is $\mathfrak{s l}_{2}^{(i)}$-integrable. Therefore $\mathcal{L}_{p} T_{i}(L(\lambda))=$ 0 for $p \neq 1$ and $\operatorname{ch} \mathcal{L}_{1} T_{i}(L(\lambda))=\operatorname{ch} L(\lambda)$ by Proposition 5.2 ,

\subsection{Twisting functors associated with integral Weyl group.}

Lemma 5.4. Let $\lambda \in \mathfrak{h}^{*}, \alpha \in \Pi(\lambda)$. There exists $x \in \mathcal{W}$ and $\alpha_{i} \in \Pi$ such that $s_{\alpha}=x s_{i} x^{-1}, \ell\left(s_{\alpha}\right)=2 \ell(x)+1$ and $\Delta_{+}^{r e} \cap x\left(\Delta_{-}^{r e}\right) \cap \Delta(\lambda)=\emptyset$.

Proof. Let $s_{\alpha}=s_{j_{l}} s_{j_{l-1}} \ldots s_{j_{1}}$ be a reduced expression of $s_{\alpha}$ in $\mathcal{W}$. Then

$$
\Delta_{+}^{r e} \cap s_{\alpha}\left(\Delta_{-}^{r e}\right)=\left\{\alpha_{1}, s_{j_{1}}\left(\alpha_{j_{2}}\right), \ldots, s_{j_{1}} \ldots s_{j_{l-1}}\left(\alpha_{j_{l}}\right)\right\}
$$

Since $\ell_{\lambda}(\alpha)=1, \Delta_{+}^{r e} \cap s_{\alpha}\left(\Delta_{-}^{r e}\right) \cap \Delta(\lambda)=\{\alpha\}$. Thus there exists $r$ such that $\alpha=s_{j_{1}} \ldots s_{j_{r-1}}\left(\alpha_{j_{r}}\right)$. Set $x=s_{j_{1}} \ldots s_{j_{r-1}}, i=j_{r}$. Then $s_{\alpha}=s_{x\left(\alpha_{i}\right)}=x s_{i} x^{-1}$. It follows that $s_{j_{l}} \ldots s_{j_{r+1}}=x$ and $\ell\left(s_{\alpha}\right)=2 \ell(x)+1$. Also $\Delta_{+}^{r e} \cap s_{\alpha}\left(\Delta_{-}^{r e}\right) \cap \Delta(\lambda)=\{\alpha\}$ implies that $\Delta_{+}^{r e} \cap x\left(\Delta_{-}^{r e}\right) \cap \Delta(\lambda)=\emptyset$. 
Note that if $\lambda, \alpha, \alpha_{i}, x$ are as in Lemma 5.4, then

$$
T_{\alpha}=T_{x} \circ T_{i} \circ T_{x^{-1}} .
$$

Let $\mathcal{O}_{[\lambda]}^{\mathfrak{g}}$ be the block of $\mathcal{O}^{\mathfrak{g}}$ corresponding to $\lambda$, that is, the full subcategory of $\mathcal{O}^{\mathfrak{g}}$ consisting of objects $M$ such that $[M: L(\mu)] \neq 0 \Rightarrow \mu \in \mathcal{W}(\lambda) \circ \mu$, where $[M: L(\mu)]$ is the multiplicity of $L(\mu)$ in the local composition factor of $M$.

Lemma 5.5. Let $\lambda \in \mathfrak{h}^{*}, y \in \mathcal{W}$, and suppose that $\left\langle\lambda+\rho, \alpha^{\vee}\right\rangle \notin \mathbb{Z}$ for all $\alpha \in$ $\Delta_{+}^{r e} \cap y^{-1}\left(\Delta_{-}^{r e}\right)$. Then $T_{y} M(w \circ \lambda) \cong M(y w \circ \lambda), T_{y} L(w \circ \lambda) \cong L(y w \circ \lambda)$ for $w \in \mathcal{W}(\lambda)$. Moreover, $T_{w}$ gives an equivalence of categories $\mathcal{O}_{[\lambda]}^{\mathfrak{g}} \stackrel{\sim}{\rightarrow} \mathcal{O}_{[w \circ \lambda]}^{\mathfrak{g}}$. The same is true for $G_{w}$.

Proof. First note that the assumption implies that $\mathcal{W}(y \circ \lambda)=y \mathcal{W}(\lambda) y^{-1}$.

We prove by induction on $\ell(y)$. Let $\ell(y)=1$, so that $y=s_{i}$ for $i \in I$. Then the fact that $T_{i} M(w \lambda) \cong M\left(s_{i} w \circ \lambda\right)$ with $w \in \mathcal{W}(\lambda)$ follow from (24). By [A1, Theorems 3.1, 3.2] any object of $\mathcal{O}_{[\lambda]}^{\mathfrak{g}}$ and $\mathcal{O}_{\left[s_{i} \circ \lambda\right]}^{\mathfrak{g}}$ is free over $\mathbb{C}\left[f_{i}\right]$ and cofree over $\mathbb{C}\left[e_{i}\right]$. Hence by Lemma 4.1 $T_{i}$ gives an equivalence of categories $\mathcal{O}_{[\lambda]}^{\mathfrak{g}} \stackrel{\sim}{\rightarrow} \mathcal{O}_{\left[s_{i} \circ \lambda\right]}^{\mathfrak{g}}$ with a quasi-inverse $G_{i}$. It follows that $T_{i} L(\lambda)$ is a simple $\mathfrak{g}$-module which is a quotient of $T_{i} M(\lambda)=M\left(s_{i} \circ \lambda\right)$, and hence is isomorphic to $L\left(s_{i} \circ \lambda\right)$. Next let $y=s_{i} z$ with $z \in \mathcal{W}, \ell(y)=\ell(z)+1$. Then $\Delta_{+}^{r e} \cap y^{-1}\left(\Delta_{-}^{r e}\right)=\left\{z^{-1}\left(\alpha_{i}\right)\right\} \sqcup\left(\Delta_{+}^{r e} \cap z^{-1} \Delta_{-}^{r e}\right)$. The assertion follows from the induction hypothesis.

Corollary 5.6. Let $\lambda, \alpha, \alpha_{i}, x$ be as in Lemma 5.4. Then $T_{x}$ give an equivalence of categories $\mathcal{O}_{\left[x^{-1} \circ \lambda\right]}^{\mathfrak{g}} \stackrel{\sim}{\rightarrow} \mathcal{O}_{[\lambda]}^{\mathfrak{g}}$ such that $T_{x} M(\mu) \cong M(x \circ \mu), T_{x} L(\mu) \cong M(x \circ \mu)$ for $\mu \in \mathcal{W}\left(x^{-1} \circ \lambda\right) \circ x^{-1} \lambda=x^{-1} \mathcal{W}(\lambda) \circ \lambda$.

Lemma 5.7. Let $\lambda \in \mathfrak{h}^{*}, \alpha_{i} \in \Pi$ such that $\left\langle\lambda+\rho, \alpha_{i}^{\vee}\right\rangle \notin \mathbb{Z}$. Then $T_{i} M^{w}(\lambda) \cong$ $M^{s_{i} w s_{i}}\left(s_{i} \circ \lambda\right)$ for $w \in \mathcal{W}(\lambda)$.

Proof. By Lemma 5.5, $T_{i} M^{w}(\lambda) \cong T_{i} T_{w} M\left(w^{-1} \circ \lambda\right) \cong T_{i} T_{w} T_{i} M\left(s_{i} w^{-1} \circ \lambda\right) \cong$ $T^{s_{i} w s_{i}} M\left(s_{i} w^{-1} s_{i} s_{i} \circ \lambda\right)$.

Lemma 5.8. Let $\lambda \in \mathfrak{h}^{*}, \alpha_{i} \in \Pi$ such that $\left\langle\lambda+\rho, \alpha_{i}^{\vee}\right\rangle \notin \mathbb{Z}$. Then $T_{i}^{2}: \mathcal{O}_{[\lambda]}^{\mathfrak{g}} \rightarrow \mathcal{O}_{[\lambda]}^{\mathfrak{g}}$ is isomorphic to the identity functor, and so is $G_{i}^{2}: \mathcal{O}_{[\lambda]}^{\mathfrak{g}} \rightarrow \mathcal{O}_{[\lambda]}^{\mathfrak{g}}$.

Proof. By Lemma $5.5 T_{i}^{2}$ induces an auto-equivalence of the category $\mathcal{O}_{[\lambda]}^{\mathfrak{g}}$ such that $T_{i}^{2} M(w \circ \lambda) \cong M(w \circ \lambda)$ and $T_{i}^{2}(L(w \circ \lambda)) \cong L(w \circ \lambda)$ for all $w \in \mathcal{W}(\lambda)$. The standard argument shows that such a functor must be isomorphic to the identify functor.

Corollary 5.9. Let $\lambda \in \mathfrak{h}^{*}, w=s_{\alpha} y \in \mathcal{W}(\lambda), \alpha \in \Pi(\lambda), y \in \mathcal{W}(\lambda), \ell_{\lambda}(w)=$ $\ell_{\lambda}(y)+1$. Then $T_{w}: \mathcal{O}_{[\lambda]}^{\mathfrak{g}} \rightarrow \mathcal{O}_{[w \circ \lambda]}^{\mathfrak{g}}$ is isomorphic to the functor $T_{s_{\alpha}} \circ T_{y}: \mathcal{O}_{[\lambda]}^{\mathfrak{g}} \rightarrow$ $\mathcal{O}_{[w \circ \lambda]}^{\mathfrak{g}}$.

Proposition 5.10. Let $\lambda \in \mathfrak{h}^{*}, w \in \mathcal{W}(\lambda), \alpha \in \Pi(\lambda)$ and suppose that $\langle w(\lambda+$ $\left.\rho), \alpha^{\vee}\right\rangle \notin \mathbb{N}$. Then the following sequence is exact:

$$
0 \rightarrow M\left(s_{\alpha} w \circ \lambda\right) \stackrel{\varphi_{1}}{\rightarrow} M(w \circ \lambda) \stackrel{\varphi_{2}}{\rightarrow} M^{s_{\alpha}}(w \circ \lambda) \stackrel{\varphi_{3}}{\rightarrow} M^{s_{\alpha}}\left(s_{\alpha} w \circ \lambda\right) \rightarrow 0,
$$

where $\varphi_{1}, \varphi_{2}, \varphi_{3}$ are any non-trivial $\mathfrak{g}$-homomorphisms. 
Proof. First observe that $\operatorname{Hom}_{\mathfrak{g}}\left(M\left(s_{\alpha} w \circ \lambda\right), M(w \circ \lambda)\right), \operatorname{Hom}_{\mathfrak{g}}\left(M(w \circ \lambda), M^{s_{\alpha}}(w \circ \lambda)\right)$ and $\operatorname{Hom}_{\mathfrak{g}}\left(M^{s_{\alpha}}(w \circ \lambda), M^{s_{\alpha}}\left(s_{\alpha} w \circ \lambda\right)\right)$ are all one-dimensional. (The first and the third are one-dimensional by Theorem 4.2.) By Lemma 5.4 there exists $x \in \mathcal{W}$ and $\alpha_{i} \in \Pi$ such that $s_{\alpha}=x s_{i} x^{-1}, \ell\left(s_{\alpha}\right)=2 \ell(x)+1$, and $\Delta_{+}^{r e} \cap x\left(\Delta_{-}^{r e}\right) \cap \Delta(\lambda)=\emptyset$. We have

$$
\begin{aligned}
M(y \circ \lambda) & \cong T_{x} M\left(x^{-1} y \circ \lambda\right), \\
M^{s_{\alpha}}(y \circ \lambda) & =T_{x} T_{i} T_{x^{-1}} M\left(x s_{i} x^{-1} y \circ \lambda\right) \\
& \cong T_{x} T_{i} M\left(s_{i} x^{-1} y \circ \lambda\right) \cong T_{x} M^{s_{i}}\left(x^{-1} y \circ \lambda\right)
\end{aligned}
$$

for $y \in \mathcal{W}(\lambda)$ by Lemma [5.5. Since $\left\langle x^{-1} w(\lambda+\rho), \alpha_{i}^{\vee}\right\rangle=\left\langle w(\lambda+\rho), \alpha^{\vee}\right\rangle \in \mathbb{N}$ there is an exact sequence

$$
0 \rightarrow M\left(s_{i} x^{-1} w \circ \lambda\right) \rightarrow M\left(x^{-1} w \circ \lambda\right) \rightarrow M^{s_{i}}\left(x^{-1} w \circ \lambda\right) \rightarrow M^{s_{i}}\left(s_{i} x^{-1} w \circ \lambda\right) \rightarrow 0
$$

by [AL, Propostion 6.2]. The required exact sequence is obtained by applying the exact functor $T_{x}: \mathcal{O}_{\left[x^{-1} \circ \lambda\right]}^{\mathfrak{g}} \rightarrow \mathcal{O}_{[\lambda]}^{\mathfrak{g}}$ to the above.

Proposition 5.11. Let $\lambda \in \mathfrak{h}^{*}, \alpha \in \Pi(\lambda), M \in \mathcal{O}_{[\lambda]}^{\mathfrak{g}}$. Take $\alpha_{i} \in \Pi, x \in \mathcal{W}$ such that $\alpha=x\left(\alpha_{i}\right)$ and $x^{-1} \Delta(\lambda)_{+} \subset \Delta_{+}^{r e}$ as in Lemma 5.4. Let $N^{\prime}$ be the largest $\mathfrak{s l}_{2}^{(i)}$-integrable submodule of $T_{x^{-1}}(M)$ and set $N=T_{x}\left(N^{\prime}\right) \subset M$. Then $T_{\alpha}(M) \cong T_{s_{\alpha}}(M / N), \operatorname{ch} \mathcal{L}_{1} T_{s_{\alpha}}(M)=\operatorname{ch} N$ and $\mathcal{L}_{p} T_{s_{\alpha}}(M)=0$ for $p \geq 2$.

Proof. We have $T_{\alpha}=T_{x} T_{i} T_{x^{-1}}$ and $T_{x^{-1}}: \mathcal{O}_{[\lambda]}^{\mathfrak{g}} \rightarrow \mathcal{O}_{\left[x^{-1} \circ \lambda\right]}^{\mathfrak{g}}, T_{x}: \mathcal{O}_{x^{-1} \circ \lambda}^{\mathfrak{g}} \rightarrow \mathcal{O}_{[\lambda]}^{\mathfrak{g}}$ are exact functors by Corollary 5.6. Therefore

$$
\mathcal{L}_{p} T_{s_{\alpha}}(M)=T_{x}\left(\mathcal{L}_{p} T_{i}\left(T_{x^{-1}} M\right)\right) .
$$

Hence Proposition 5.2 gives that

$$
\begin{aligned}
& T_{s_{\alpha}}(M)=T_{x} T_{i} T_{x^{-1}}(M) \cong T_{x} T_{i}\left(T_{x^{-1}}(M) / N^{\prime}\right) \cong T_{x} T_{i} T_{x^{-1}}(M / N)=T_{s_{\alpha}}(M / N), \\
& \operatorname{ch} \mathcal{L}_{1} T_{s_{\alpha}}(M)=\operatorname{ch} T_{x} T_{x^{-1}}(N)=\operatorname{ch} N, \\
& \mathcal{L}_{p} T_{s_{\alpha}}(M)=0 \quad \text { for } p \geq 0 .
\end{aligned}
$$

This completes the proof.

Theorem 5.12. Let $\lambda \in \mathfrak{h}^{*}$ be regular dominant weight, $w \in \mathcal{W}(\lambda)$. Then

$$
\mathcal{L}_{p} T_{w}(L(\lambda)) \cong \begin{cases}L(\lambda) & \text { if } p=\ell(w) \\ 0 & \text { otherwise }\end{cases}
$$

Proof. Let $\alpha \in \Pi(\lambda)$. Since $T_{x^{-1}} L(\lambda)=L\left(x^{-1} \circ \lambda\right)$ and $\left\langle x^{-1} \circ \lambda+\rho, \alpha_{i}^{\vee}\right\rangle=$ $\left\langle\lambda+\rho, \alpha^{\vee}\right\rangle \in \mathbb{N}, T_{x^{-1}} L(\lambda)$ is $\mathfrak{s l}_{2}^{(i)}$-integrable. Thus,

$$
\mathcal{L}_{p} T_{i} T_{x^{-1}} L(\lambda) \cong \begin{cases}T_{x^{-1}} L(\lambda) & \text { if } p=1 \\ 0 & \text { if } p \neq 0\end{cases}
$$

by Theorem 5.3. It follows from (37) that

$$
\mathcal{L}_{p} T_{s_{\alpha}}(L(\lambda)) \cong \begin{cases}L(\lambda) & \text { if } p=1, \\ 0 & \text { otherwise. }\end{cases}
$$

Finally, the assertion follows in the same manner as in [AS, Corollary 6.2] by Corollary 5.9 
6. Two-SIDED BGG RESOLUTIONS OF ADMISSIBLE REPRESENTATIONS

6.1. Admissible representations. A weight $\lambda \in \mathfrak{h}^{*}$ is called admissible if it is regular dominant and

$$
\mathbb{Q} \Delta(\lambda)=\mathbb{Q} \Delta^{r e} .
$$

The irreducible representation $L(\lambda)$ is called admissible if $\lambda$ is admissible. A complex number $k$ is called an admissible number for $\mathfrak{g}$ if the weight $k \Lambda_{0}$ is admissible.

Let $r^{\vee}$ be the lacing number of $\stackrel{\circ}{\mathfrak{g}}$, that is, the maximal number of the edges of the Dynkin diagram of $\stackrel{\circ}{\mathfrak{g}}$. Also, let $h$ be the Coxeter number of $\stackrel{\circ}{\mathfrak{g}}$.

Proposition 6.1 ([KW2, $[\mathrm{KW}])$. A complex number $k$ is admissible if and only if

$$
k+h^{\vee}=\frac{p}{q} \quad \text { with } p, q \in \mathbb{N},(p, q)=1, p \geq\left\{\begin{array}{ll}
h^{\vee} & \text { if }\left(r^{\vee}, q\right)=1, \\
h & \text { if }\left(r^{\vee}, q\right)=r^{\vee}
\end{array} .\right.
$$

A complex number $k$ of the form (39) is called an admissible number with denominator $q$. For an an admissible number $k$ with denominator $q$, we have

$\Delta\left(k \Lambda_{0}\right)=\{\alpha+n q \delta ; \alpha \in \Delta, n \in \mathbb{Z}\} \cong \Delta^{r e}$ and $\mathcal{W}\left(k \Lambda_{0}\right) \cong \mathcal{W}$ if $\left(r^{\vee}, q\right)=1$, $\Delta\left(k \Lambda_{0}\right)^{\vee}=\left\{\alpha^{\vee}+n q \delta ; \alpha \in \Delta, n \in \mathbb{Z}\right\} \cong{ }^{L} \Delta^{r e}$ and $\mathcal{W}\left(k \Lambda_{0}\right) \cong{ }^{L} \mathcal{W}$ if $\left(r^{\vee}, q\right)=r^{\vee}$,

where $\Delta(\lambda)^{\vee}=\left\{\alpha^{\vee} ; \alpha \in \Delta(\lambda)\right\}$ and ${ }^{L} \Delta^{r e}$ and ${ }^{L} \mathcal{W}$ are the real root system and the Weyl group of the non-twisted affine Kac-Moody algebra ${ }^{L} \mathfrak{g}$ associated with the Langlands dual $L_{\mathfrak{g}}^{\circ}$ of $\stackrel{\circ}{\mathfrak{g}}$, respectively. Set

$$
\dot{\alpha_{0}}= \begin{cases}-\theta+q \delta & \text { if }\left(r^{\vee}, q\right)=1, \\ -\theta_{s}+\frac{q}{r^{\vee}} \delta & \text { if }\left(r^{\vee}, q\right)=r^{\vee} .\end{cases}
$$

Then $\Pi\left(k \Lambda_{0}\right)=\left\{\alpha_{1}, \ldots, \alpha_{\ell}, \dot{\alpha}_{0}\right\}$. Put $\dot{s}_{0}=s_{\dot{\alpha}_{0}} \in \mathcal{W}\left(k \Lambda_{0}\right)$, so that $\mathcal{W}\left(k \Lambda_{0}\right)=$ $\left\langle s_{1}, \ldots, s_{\ell}, \dot{s}_{0}\right\rangle$.

For an admissible number $k$ let $\operatorname{Pr}_{k}^{+}$be the set of admissible weights $\lambda$ of level $k$ such that $\lambda\left(\alpha^{\vee}\right) \in \mathbb{Z}_{\geq 0}$ for all $\alpha \in \stackrel{\circ}{\Delta}_{+}$. Then $\left\{L(\lambda) ; \lambda \in \operatorname{Pr}_{k}^{+}\right\}$is the set of irreducible admissible representations of level $k$ which are integrable over $\stackrel{\circ}{\mathfrak{g}} \subset \mathfrak{g}$. We have $\Delta(\lambda)=\Delta\left(k \Lambda_{0}\right)$ for $\lambda \in P r_{k}^{+}$.

For an admissible number $k$ denote by $\operatorname{Pr}_{k}$ the set of admissible weights $\lambda$ of level $k$ such that $\Delta(\lambda) \cong \Delta\left(k \Lambda_{0}\right)$ as root systems. Then KW2

$$
\operatorname{Pr}_{k}=\bigcup_{\substack{y \in \mathcal{W} e \\ y\left(\Delta\left(k \Lambda_{0}\right) \subset \Delta_{+}^{r e}\right.}} \operatorname{Pr}_{k, y}, \quad \operatorname{Pr}_{k, y}=y \circ \operatorname{Pr}_{k}^{+} .
$$

Note that

$$
\mathcal{W}(\lambda)=y \mathcal{W}\left(k \Lambda_{0}\right) y^{-1} \quad \text { for } \lambda \in \operatorname{Pr}_{k, y} .
$$

For $\lambda \in P r_{k}$, let $\ell_{\lambda}^{\frac{\infty}{2}}(?)$ be the semi-infinite length function of the affine Weyl group $\mathcal{W}(\lambda)$. The semi-infinite Bruhat ordering $\preceq_{\lambda, \frac{\infty}{2}}$ are also defined for $\mathcal{W}(\lambda)$. We will use the symbol $w \triangleright_{\lambda, \frac{\infty}{2}} w^{\prime}$ to denote a covering in the twisted Bruhat order $\succeq_{\lambda, \frac{\infty}{2}}$.

Remark 6.2. The admissible weight $\lambda \in P r_{k}$ is called the principal admissible weight KW2 if $\Delta(\lambda) \cong \Delta^{r e}$, that is, if the denominator $q$ of $k$ is prime to $r^{\vee}$. 


\subsection{Fiebig's equivalence and BGG resolution of admissible representa-}

tions. The following theorem is the special case of a result of Fiebig [Fie, Theorem $11]$.

Theorem 6.3 ([Fie]). Let $\lambda$ be regular dominant. Suppose that there exists a symmetrizable Kac-Moody algebra $\mathfrak{g}^{\prime}$ whose Weyl group $\mathcal{W}^{\prime}$ is isomorphic to $\mathcal{W}(\lambda)$. Let $\lambda^{\prime}$ be an integral dominant weight of $\mathfrak{g}^{\prime}, \mathcal{O}_{\left[\lambda^{\prime}\right]}^{\mathfrak{g}^{\prime}}$ the block of $\mathcal{O}^{\mathfrak{g}^{\prime}}$ containing the irreducible highest weight representation $L^{\mathfrak{g}^{\prime}}\left(\lambda^{\prime}\right)$ of $\mathfrak{g}$ with highest weight $\lambda^{\prime}$. Then there is an equivalence of categories

$$
\mathcal{O}_{[\lambda]}^{\mathfrak{g}} \cong \mathcal{O}_{\left[\lambda^{\prime}\right]}^{\mathfrak{g}^{\prime}}
$$

which maps $M(w \circ \lambda)$ and $L(w \circ \lambda), w \in \mathcal{W}(\lambda)$, to $M^{\mathfrak{g}^{\prime}}\left(\phi(w) \circ \lambda^{\prime}\right)$ and $L^{\mathfrak{g}}\left(\phi(w) \circ \lambda^{\prime}\right)$, respectively. Here $M^{\mathfrak{g}^{\prime}}\left(\lambda^{\prime}\right)$ is the Verma module of $\mathfrak{g}^{\prime}$ with highest weight $\lambda^{\prime}$ and $\phi: \mathcal{W}(\lambda) \stackrel{\sim}{\rightarrow} \mathcal{W}^{\prime}$ is the isomorphism.

Let $k$ be an admissible number with denominator $q, \lambda \in P r_{k}$. By Theorem 6.3 the block $\mathcal{O}_{[\lambda]}^{\mathfrak{g}}$ is equivalent to a block of the category $\mathcal{O}$ of $\mathfrak{g}$ or ${ }^{L} \mathfrak{g}$ containing an integrable representation. In particular the existence of a BGG resolution of an integrable representation of an affine Kac-Moody algebra [GL, RCW] implies the existence of a BGG resolution for $L(\lambda)$ :

Theorem 6.4. Let $k$ be an admissible number, $\lambda \in P r_{k}$. Then there exists a complex

$$
\text { B. }(\lambda): \cdots \stackrel{d_{3}}{\rightarrow} \mathcal{B}_{2}(\lambda) \stackrel{d_{2}}{\rightarrow} \mathcal{B}_{1}(\lambda) \stackrel{d_{1}}{\rightarrow} \mathcal{B}_{0}(\lambda) \stackrel{d_{0}}{\rightarrow} 0
$$

of the form $\mathcal{B}_{i}(\lambda)=\bigoplus_{\substack{w \in \mathcal{W}(\lambda) \\ \ell_{\lambda}(w)=i}} M(w \circ \lambda), d_{i}=\sum_{\substack{w, w^{\prime} \in \mathcal{W}(\lambda) \\ \ell_{\lambda}(w)=i, w \triangleright{ }_{\lambda} w^{\prime}}} d_{w^{\prime}, w}, d_{w^{\prime}, w} \in \operatorname{Hom}_{\mathfrak{g}}(M(w \circ$ $\left.\lambda), M\left(w^{\prime} \circ \lambda\right)\right)$, such that

$$
H_{i}\left(\mathcal{B}_{\bullet}(\lambda)\right) \cong \begin{cases}L(\lambda) & \text { if } i=0, \\ 0 & \text { otherwise } .\end{cases}
$$

The resolution of $L(\lambda)$ in Theorem 6.4 can be combinatorially constructed as follows BGG]: Fix a $\mathfrak{g}$-homomorphisms

$$
i_{w^{\prime}, w}^{\lambda}: M(w \circ \lambda) \rightarrow M\left(w^{\prime} \circ \lambda\right)
$$

for $w, w^{\prime} \in \mathcal{W}(\lambda)$ with $w \succeq_{\lambda} w^{\prime}$ in such a way that $i_{w^{\prime \prime}, w^{\prime}}^{\lambda} \circ i_{w^{\prime}, w}^{\lambda}=i_{w^{\prime \prime}, w}^{\lambda}$ if $w \succeq_{\lambda} w^{\prime} \succeq_{\lambda} w$.

A quadruple $\left(w_{1}, w_{2}, w_{3}, w_{4}\right)$ in $\mathcal{W}(\lambda)$ is called a square if $w_{1} \triangleright_{\lambda} w_{2} \triangleright_{\lambda} w_{4}$, $w_{1} \triangleright_{\lambda} w_{3} \triangleright_{\lambda} w_{4}$ and $w_{2} \neq w_{3}$.

Theorem 6.5. Let $k$ be an admissible number, $\lambda \in \operatorname{Pr}_{k}$. Assign $\epsilon_{w_{2}, w_{1}} \in \mathbb{C}^{*}$ for every pair $\left(w_{1}, w_{2}\right)$ in $\mathcal{W}(\lambda)$ with $w_{1} \triangleright_{\lambda} w_{2}$ in such a way that $\epsilon_{w_{4}, w_{2}} \epsilon_{w_{2}, w_{1}}+$ $\epsilon_{w_{4}, w_{3}} \epsilon_{w_{3}, w_{1}}=0$ for every square $\left(w_{1}, w_{2}, w_{3}, w_{4}\right)$ of $\mathcal{W}(\lambda)$ (such an assignment is possible by [BGG] $)$. Set $d_{w^{\prime}, w}=\epsilon_{w^{\prime}, w} i_{w^{\prime}, w}^{\lambda}, d_{i}=\sum_{\substack{w, w^{\prime} \in \mathcal{W}(\lambda) \\ \ell_{\lambda}(w)=i, w \triangleright_{\lambda} w^{\prime}}} d_{w^{\prime}, w}$. Then

$$
\mathcal{B}_{\bullet}(\lambda): \cdots \stackrel{d_{3}}{\rightarrow} \mathcal{B}_{2}(\lambda) \stackrel{d_{2}}{\rightarrow} \mathcal{B}_{1}(\lambda) \stackrel{d_{1}}{\rightarrow} \mathcal{B}_{0}(\lambda) \stackrel{d_{0}}{\rightarrow} 0,
$$

where $\mathcal{B}_{i}(\lambda)=\bigoplus_{\substack{w \in \mathcal{W}(\lambda) \\ \ell_{\lambda}(w)=i}} M(w \circ \lambda)$, is a resolution of $L(\lambda)$. 
6.3. Twisted BGG resolution. For $w_{1}, w_{2}, y \in \mathcal{W}(\lambda)$ with $w_{1} \succeq_{y} w_{2}$, set

$$
\varphi_{w_{2}, w_{1}}^{\lambda, y}=T_{y}\left(i_{y^{-1} w_{2}, y^{-1} w_{1}}^{\lambda}\right): M^{y}\left(w_{1} \circ \lambda\right) \rightarrow M^{y}\left(w_{2} \circ \lambda\right) .
$$

A quadruple $\left(w_{1}, w_{2}, w_{3}, w_{4}\right)$ in $\mathcal{W}(\lambda)$ is called a $y$-twisted square if $w_{1} \triangleright_{y} w_{2} \triangleright_{y} w_{4}$, $w_{1} \triangleright_{y} w_{3} \triangleright_{y} w_{4}$ and $w_{2} \neq w_{3}$.

Theorem 6.6. Let $k$ be an admissible number, $\lambda \in P r_{k}, y \in \mathcal{W}(\lambda)$. Assign $\epsilon_{w_{2}, w_{1}}^{y} \in \mathbb{C}^{*}$ for every pair $\left(w_{1}, w_{2}\right)$ with $w_{1} \triangleright_{\lambda, y} w_{2}$ in $\mathcal{W}(\lambda)$ in such a way that $\epsilon_{w_{4}, w_{2}}^{y} \epsilon_{w_{2}, w_{1}}^{y}+\epsilon_{w_{4}, w_{3}}^{y} \epsilon_{w_{3}, w_{1}}^{y}=0$ for every $y$-twisted square $\left(w_{1}, w_{2}, w_{3}, w_{4}\right)$ of $\mathcal{W}(\lambda)$. Set

$$
\begin{aligned}
\mathcal{B}_{i}^{y}(\lambda) & =\bigoplus_{\substack{w \in \mathcal{W}(\lambda) \\
\ell_{\lambda}^{y}(w)=i}} M^{y}(w \circ \lambda), d_{w^{\prime}, w}^{y}=\epsilon_{w^{\prime}, w}^{y} \varphi_{w^{\prime}, w}^{\lambda, y}, d_{i} \\
& =\sum_{\substack{w, w^{\prime} \in \mathcal{W}(\lambda) \\
\ell_{\lambda}^{y}(w)=i, w \triangleright \triangleright_{\lambda, y^{\prime}}}} d_{w^{\prime}, w}: \mathcal{B}_{i}^{y}(\lambda) \rightarrow \mathcal{B}_{i-1}^{y}(\lambda) .
\end{aligned}
$$

Then

$$
\mathcal{B}_{\bullet}^{y}(\lambda): \cdots \stackrel{d_{3}}{\rightarrow} \mathcal{B}_{2}^{y}(\lambda) \stackrel{d_{2}}{\rightarrow} \mathcal{B}_{1}^{y}(\lambda) \stackrel{d_{1}}{\rightarrow} \mathcal{B}_{0}^{y}(\lambda) \stackrel{d_{0}}{\rightarrow} \mathcal{B}_{-1}^{y}(\lambda) \rightarrow \ldots \rightarrow \mathcal{B}_{-\ell(y)}^{y}(\lambda) \rightarrow 0
$$

is a complex of $\mathfrak{g}$-modules such that

$$
H_{i}\left(B_{\bullet}^{y}(\lambda)\right) \cong \begin{cases}L(\lambda) & \text { for } i=0 \\ 0 & \text { otherwise. }\end{cases}
$$

Proof. Set $\epsilon_{y^{-1} w_{1}, y^{-1} w_{2}}=\epsilon_{w_{1}, w_{2}}^{y}$. Then $\left\{\epsilon_{w_{1}, w_{2}}^{y}\right\}$ satisfies the condition in Theo-

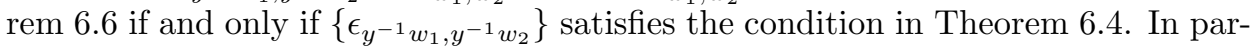
ticular such an assignment is possible. Consider the BGG resolution $\mathcal{B}_{\bullet}(\lambda)$ of $L(\lambda)$ in Theorem 6.5 associated with this assignment. We have $\mathcal{B}_{\bullet}^{y}(\lambda)=T_{y}\left(B_{\bullet}(\lambda)\right)[-\ell(y)]$, where $[-\ell(y)]$ denotes the shift of the degree. Therefore the assertion follows from Theorem 5.12 .

\subsection{System of twisted BGG resolutions.}

Proposition 6.7. Let $\lambda \in \mathfrak{h}^{*}$ be regular dominant, $y=s_{\beta_{1}} s_{\beta_{2}} \ldots s_{\beta_{l}}$ a reduced expression of $y \in \mathcal{W}(\lambda)$ with $\beta_{i} \in \Pi(\lambda)$. Set $y_{i}=s_{\beta_{1}} s_{\beta_{2}} \ldots s_{\beta_{i}}$ for $i=0,1, \ldots, l$ and fix a non-zero $\mathfrak{g}$-homomorphism $\phi_{w}^{y_{i}}: M^{y_{i}}(w \circ \lambda) \rightarrow M^{y_{i+1}}(w \circ \lambda)$ for $w \in \mathcal{W}(\lambda)$, $i=1, \ldots, l$. One can assign $\epsilon_{w_{2}, w_{1}}^{i} \in \mathbb{C}^{*}$ for each pair $\left(w_{1}, w_{2}\right)$ with $w_{1} \triangleright_{\lambda, y_{i}} w_{2}$ for all $i=1, \ldots, l$ in such a way that the following hold:

(i) $\epsilon_{w_{4}, w_{2}}^{i} \epsilon_{w_{2}, w_{1}}^{i}+\epsilon_{w_{4}, w_{3}}^{i} \epsilon_{w_{3}, w_{1}}^{i}=0$ for every $y_{i}$-twisted square $\left(w_{1}, w_{2}, w_{3}, w_{4}\right)$ of $\mathcal{W}(\lambda)$,

(ii) If $w_{1} \triangleright_{\lambda, y_{i}} w_{2}, w_{1} \triangleright_{\lambda, y_{i-1}} w_{2}, \ell_{\lambda}^{y_{i}}\left(w_{1}\right)=\ell_{\lambda}^{y_{i-1}}\left(w_{1}\right)$ and $\ell_{\lambda}^{y_{i}}\left(w_{2}\right)=\ell_{\lambda}^{y_{i-1}}\left(w_{2}\right)$, then the the following diagram commutes:

$$
\begin{array}{cc}
M^{y_{i-1}}\left(w_{1} \circ \lambda\right) \stackrel{\epsilon_{w_{2}, w_{1}} \varphi_{w_{2}, w_{1}}^{\lambda, y_{i-1}}}{\longrightarrow} & M^{y_{i-1}}\left(w_{2} \circ \lambda\right) \\
\phi_{w_{1}}^{y_{i-1}} \downarrow & \downarrow \phi_{w_{2}}^{y_{i-1}} \\
M^{y}\left(w_{1} \circ \lambda\right) \stackrel{\epsilon_{w_{2}, w_{1}}^{i} \varphi_{w_{2}, w_{1}}^{\lambda, y_{i}}}{\longrightarrow} & M^{y}\left(w_{2} \circ \lambda\right) .
\end{array}
$$

Proposition 6.8. Let $\lambda \in \mathfrak{h}^{*}$ be regular dominant, $y \in \mathcal{W}(\lambda), \alpha \in \Pi(\lambda)$ such that $\ell_{\lambda}\left(y s_{\alpha}\right)=\ell_{\lambda}(y)+1$. Set $\beta=y(\alpha)$. 
(i) Let $w_{1}, w_{2} \in \mathcal{W}(\lambda)$. Suppose that $w_{1} \triangleright_{y} w_{2}, w_{1} \triangleright_{y s_{\alpha}} w_{2}$ and $\ell_{\lambda}^{y}\left(w_{1}\right)=$ $\ell_{\lambda}^{y s_{\alpha}}\left(w_{1}\right)$. Then

$$
\operatorname{dim}_{\mathbb{C}} \operatorname{Hom}_{\mathfrak{g}}\left(M^{y}\left(w_{1} \circ \lambda\right), M^{y s_{\alpha}}\left(w_{2} \circ \lambda\right)\right)=1 .
$$

Moreover, either of the followings span the one-dimensional vector space $\operatorname{Hom}_{\mathfrak{g}}\left(M^{y}\left(w_{1} \circ \lambda\right), M^{y s_{\alpha}}\left(w_{2} \circ \lambda\right)\right)$ :

(a) the composition $M^{y}\left(w_{1} \circ \lambda\right) \rightarrow M^{y}\left(w_{2} \circ \lambda\right) \rightarrow M^{y s_{\alpha}}\left(w_{2} \circ \lambda\right)$ of any non-trivial $\mathfrak{g}$-homomorphisms;

(b) the composition $M^{y}\left(w_{1} \circ \lambda\right) \rightarrow M^{y s_{\alpha}}\left(w_{1} \circ \lambda\right) \rightarrow M^{y s_{\alpha}}\left(w_{2} \circ \lambda\right)$ of any non-trivial $\mathfrak{g}$-homomorphisms.

(ii) Let $w_{1}, w_{2} \in \mathcal{W}(\lambda)$. Suppose that $\ell_{\lambda}^{y}\left(w_{1}\right)=\ell_{\lambda}^{y}\left(w_{2}\right)+2$ and $w_{i}^{-1}(\beta) \in \Delta_{+}^{r e}$ for $i=1,2$. Then the composition $M^{y}\left(w_{1} \circ \lambda\right) \rightarrow M^{y}\left(w_{2} \circ \lambda\right) \rightarrow M^{y s_{\alpha}}\left(w_{2} \circ\right.$ $\lambda)$ of any non-trivial homomorphisms is non-zero.

(iii) Let $w \in \mathcal{W}(\lambda)$ and suppose that $s_{\alpha} w \triangleright_{\lambda, y} w$. Then the composition $M^{y}\left(s_{\alpha} w \circ\right.$ $\lambda) \rightarrow M^{y}(w \circ \lambda) \rightarrow M^{y s_{\alpha}}(w \circ \lambda)$ of any $\mathfrak{g}$-homomorphisms is zero.

Proof. (i) Since $y^{-1} w_{1} \triangleright y^{-1} w_{2}$, the Jantzen sum formula implies that

$$
\left[M\left(y^{-1} w_{2} \circ \lambda\right): L\left(y^{-1} w_{1} \circ \lambda\right)\right]=1 .
$$

Hence $\left[M^{s_{\alpha}}\left(y^{-1} w_{2} \circ \lambda\right): L\left(y^{-1} w_{1} \circ \lambda\right)\right]=1$. As

$$
\operatorname{Hom}\left(M^{y}\left(w_{1} \circ \lambda\right), M^{y s_{\alpha}}\left(w_{2} \circ \lambda\right)\right) \cong \operatorname{Hom}\left(M\left(y^{-1} w_{1} \circ \lambda\right), M^{s_{\alpha}}\left(y^{-1} w_{2} \circ \lambda\right)\right),
$$

it follows that

$$
\operatorname{dim}_{\mathbb{C}} \operatorname{Hom}\left(M^{y}\left(w_{1} \circ \lambda\right), M^{y s_{\alpha}}\left(w_{2} \circ \lambda\right)\right) \leq 1 .
$$

Now we have

$$
\operatorname{Hom}_{\mathfrak{g}}\left(M^{y}\left(w_{1} \circ \lambda\right), M^{y}\left(w_{2} \circ \lambda\right)\right) \cong \operatorname{Hom}_{\mathfrak{g}}\left(M\left(y^{-1} w_{1} \circ \lambda\right), M\left(y^{-1} w_{2} \circ \lambda\right)\right),
$$$$
\operatorname{Hom}_{\mathfrak{g}}\left(M^{y}\left(w_{1} \circ \lambda\right), M^{y s_{\alpha}}\left(w_{1} \circ \lambda\right)\right) \cong \operatorname{Hom}_{\mathfrak{g}}\left(M\left(y^{-1} w_{1} \circ \lambda\right), M^{s_{\alpha}}\left(y^{-1} w_{1} \circ \lambda\right)\right) \text {, }
$$$$
\operatorname{Hom}_{\mathfrak{g}}\left(M^{y}\left(w_{2} \circ \lambda\right), M^{y s_{\alpha}}\left(w_{2} \circ \lambda\right)\right) \cong \operatorname{Hom}_{\mathfrak{g}}\left(M\left(y^{-1} w_{2} \circ \lambda\right), M^{s_{\alpha}}\left(y^{-1} w_{2} \circ \lambda\right)\right) \text {, }
$$

$\operatorname{Hom}_{\mathfrak{g}}\left(M^{y s_{\alpha}}\left(w_{1} \circ \lambda\right), M^{y s_{\alpha}}\left(w_{2} \circ \lambda\right)\right) \cong \operatorname{Hom}_{\mathfrak{g}}\left(M\left(s_{\alpha} y^{-1} w_{1} \circ \lambda\right), M\left(s_{\alpha} y^{-1} w_{2} \circ \lambda\right)\right)$.

In particular they are all one-dimensional. Hence it remains to show that the compositions in (a) and (b) are non-trivial. This is equivalent to the non-triviality of the compositions

$$
\begin{aligned}
& M\left(y^{-1} w_{1} \circ \lambda\right) \rightarrow M\left(y^{-1} w_{2} \circ \lambda\right) \rightarrow M^{s_{\alpha}}\left(y^{-1} w_{2} \circ \lambda\right) \\
& \text { and } M\left(y^{-1} w_{1} \circ \lambda\right) \rightarrow M^{s_{\alpha}}\left(y^{-1} w_{1} \circ \lambda\right) \rightarrow M^{s_{\alpha}}\left(y^{-1} w_{2} \circ \lambda\right),
\end{aligned}
$$

respectively. Therefore we may assume that $y=1$.

Since $\left\langle w_{2}(\lambda+\rho), \alpha^{\vee}\right\rangle \in \mathbb{N}$, we have the exact sequence

$$
0 \rightarrow M\left(s_{\alpha} w_{2} \circ \lambda\right) \rightarrow M\left(w_{2} \circ \lambda\right) \rightarrow M^{s_{\alpha}}\left(w_{2} \circ \lambda\right) \rightarrow M^{s_{\alpha}}\left(s_{\alpha} w_{2} \circ \lambda\right) \rightarrow 0
$$

by Proposition 5.10. On the other hand,

$$
w_{1} \circ \lambda \npreceq \lambda s_{\alpha} w_{2} \circ \lambda
$$

as we have the square $\left(s_{\alpha} w_{1}, w_{1}, s_{\alpha} w_{2}, w_{2}\right)$ by the assumption and (15). Hence (43) implies that the image of the highest weight vector of $M\left(w_{1} \circ \lambda\right)$ in $M\left(w_{2} \circ \lambda\right)$ does not lie in the kernel of the map $M\left(w_{2} \circ \lambda\right) \rightarrow M^{s_{\alpha}}\left(w_{2} \circ \lambda\right)$. This proves the 
non-triviality of the composition map in (a) for $y=1$, and thus, for all $y$. Next we show the non-triviality of the composition in (b). Consider the exact sequence

$$
0 \rightarrow M\left(s_{\alpha} w_{1} \circ \lambda\right) \rightarrow M\left(s_{\alpha} w_{2} \circ \lambda\right) \rightarrow N \rightarrow 0
$$

in the category $\mathcal{O}_{[\lambda]}^{\mathfrak{g}}$, where $N=M\left(s_{\alpha} w_{2} \circ \lambda\right) / M\left(s_{\alpha} w_{1} \circ \lambda\right)$. Applying the functor $T_{s_{\alpha}}$ we obtain the exact sequence

$$
0 \rightarrow \mathcal{L}_{1} T_{s_{\alpha}} N \rightarrow M^{s_{\alpha}}\left(w_{1} \circ \lambda\right) \rightarrow M^{s_{\alpha}}\left(w_{2} \circ \lambda\right) \rightarrow T_{i} N \rightarrow 0 .
$$

By Proposition 5.11, the weights of $\mathcal{L}_{1} T_{s_{\alpha}} N$ are contained in the set of weights of $N$, and hence of $M\left(s_{\alpha} w_{2} \circ \lambda\right)$. Therefore (44) and (45) imply that the image of the highest weight vector of $M\left(w_{1} \circ \lambda\right)$ in $M^{s_{\alpha}}\left(w_{1} \circ \lambda\right)$ does not belong to the kernel of the map $M^{s_{\alpha}}\left(w_{1} \circ \lambda\right) \rightarrow M^{s_{\alpha}}\left(w_{2} \circ \lambda\right)$. This competes the proof of (i). (ii) Similarly, as above, the problem reduces to the case $y=1$. By the assumption we have $s_{\beta} w_{1} \triangleright_{\lambda} w_{1}, s_{\beta} w_{2} \triangleright_{\lambda} w_{2}$. Thus $w_{1} \npreceq_{\lambda} s_{\beta} w_{2}$ because otherwise $\left(w_{1}, s_{\beta} w_{1}, s_{\beta} w_{1}, w_{2}\right)$ is a square. Hence (43) proves the assertion by the same argument as above. (iii) Again we may assume that $y=1$ and the assertion follows from (43).

Proof of Proposition 6.7. We prove by induction on $i$ that such an assignment is possible.

As we already remarked the case $i=0$ is the well-known result of BGG. So let $i>0$. Suppose that $w_{1} \triangleright_{\lambda, y_{i}} w_{2}$. Set $\beta=y_{i-1}\left(\alpha_{i}\right) \in \Delta_{+}^{r e}$. The following four cases are possible. (The case $w_{1}^{-1}(\beta) \in \Delta_{+}^{r e}, w_{2}^{-1}(\beta) \in \Delta_{-}^{r e}$ does not happen by [BGG, Lemma 11.3].)

I) $w_{1}^{-1}(\beta), w_{2}^{-1}(\beta) \in \Delta_{+}^{r e}$. In this case $w_{1} \triangleright_{\lambda, y_{i-1}} w_{2}, \ell_{\lambda}^{y_{i}}\left(w_{1}\right)=\ell_{\lambda}^{y_{i-1}}\left(w_{1}\right)$ and $\ell_{\lambda}^{y_{i}}\left(w_{2}\right)=\ell_{\lambda}^{y_{i-1}}\left(w_{2}\right)$. By Proposition 6.8 there exists a unique $\epsilon_{w_{2}, w_{1}}^{i}$ which makes the diagram (42) commute.

II) $w_{1}=s_{\beta} w_{2}$. In this case $w_{2} \triangleright_{\lambda, y_{i-1}} w_{1}, \ell_{\lambda}^{y_{i}}\left(w_{1}\right)=\ell_{\lambda}^{y_{i-1}}\left(w_{1}\right)-2$ and $\ell_{\lambda}^{y_{i}}\left(w_{2}\right)=$ $\ell_{\lambda}^{y_{i-1}}\left(w_{2}\right)$. We set $\epsilon_{w_{2}, w_{1}}^{i}=\epsilon_{w_{1}, w_{2}}^{i-1}$.

III) $w_{1}^{-1}(\beta), w_{2}^{-1}(\beta) \in \Delta_{-}^{r e}$. In this case $w_{1} \triangleright_{\lambda, y_{i-1}} w_{2}, \ell_{\lambda}^{y_{i}}\left(w_{1}\right)=\ell_{\lambda}^{y_{i-1}}\left(w_{1}\right)-2$ $\ell_{\lambda}^{y_{i}}\left(w_{2}\right)=\ell_{\lambda}^{y_{i-1}}\left(w_{2}\right)-2$, and we have the $y_{i}$-twisted square $\left(w_{1}, s_{\beta} w_{1}, w_{2}, s_{\beta} w_{2}\right)$. Note that $\epsilon_{s_{\beta} w_{2}, s_{\beta} w_{1}}^{i}$ is defined in I), and $\epsilon_{s_{\beta} w_{1}, w_{1}}^{i}, \epsilon_{s_{\beta} w_{2}, w_{2}}^{i}$ are defined in II). We set

$$
\epsilon_{w_{2}, w_{1}}^{i}=-\frac{\epsilon_{s_{\beta} w_{1}, w_{1}}^{i} \epsilon_{s_{\beta} w_{2}, s_{\beta} w_{1}}^{i}}{\epsilon_{s_{\beta} w_{2}, w_{2}}^{i} .}
$$

IV) $w_{1}^{-1}(\beta) \in \Delta_{-}^{r e}, w_{2}^{-1}(\beta) \in \Delta_{+}^{r e}, w_{2} \neq s_{\beta} w_{1}$. In this case there exists a unique $w_{3} \in \mathcal{W}$ such that $\left(s_{\beta} w_{1}, w_{1}, w_{3}, w_{2}\right)$ is a $y_{i}$-twisted square. Note that $w_{3}^{-1}(\beta) \in \Delta_{+}^{r e}$ because $\left(w_{3}, w_{2}, s_{\beta} w_{3}, s_{\beta} w_{2}\right)$ is a $y_{i}$-twisted square by (15). Since $\epsilon_{w_{3}, s_{\beta} w_{1}}^{i}, \epsilon_{w_{2}, w_{3}}^{i}$ are defined in I) and $\epsilon_{w_{1}, s_{\beta} w_{1}}^{i}$ is defined in II), we can set

$$
\epsilon_{w_{1}, w_{1}}^{i}=-\frac{\epsilon_{w_{3}, s_{\beta} w_{1}}^{i} \epsilon_{w_{2}, w_{3}}^{i}}{\epsilon_{w_{1}, s_{\beta} w_{1}}^{i}} .
$$

Now let $\left(w_{1}, w_{2}, w_{3}, w_{4}\right)$ be a $y_{i}$-twisted square. Set

$$
A_{i}\left(w_{1}, w_{2}, w_{3}, w_{4}\right)=\frac{\epsilon_{w_{4}, w_{2}}^{i} \epsilon_{w_{2}, w_{1}}^{i}}{\epsilon_{w_{4}, w_{3}}^{i} \epsilon_{w_{3}, w_{1}}^{i}} .
$$

We need to show that $A_{i}\left(w_{1}, w_{2}, w_{3}, w_{4}\right)=-1$.

The following four cases are possible. 
1) $w_{2}=s_{\beta} w_{1}, w_{4}=s_{\beta} w_{3}$. In this case the assertion follows from the definition (46).

2) $w_{2}=s_{\beta} w_{1}, w_{4} \neq s_{\beta} w_{3}$. In this case $\left(s_{\beta} w\right)^{-1}(\beta) \in \Delta_{-}^{r e}$, and $w_{4}^{-1}(\beta) \in \Delta_{+}^{r e}$ because otherwise $w_{3}=s_{\beta} w_{4}$. Hence the assertion follows from the definition (47).

3) $w_{2} \neq s_{\beta} w_{1}, w_{4}=s_{\beta} w_{3}$. In this case $\left(s_{\beta} w_{1}, w_{1}, s_{\beta} w_{2} . w_{2}\right),\left(s_{\beta} w_{1}, w_{1}, s_{\beta} w_{2}, w_{3}\right)$, $\left(s_{\beta} w_{2}, w_{2}, s_{3}, w_{4}\right)$ are $y_{i}$-twisted squares:

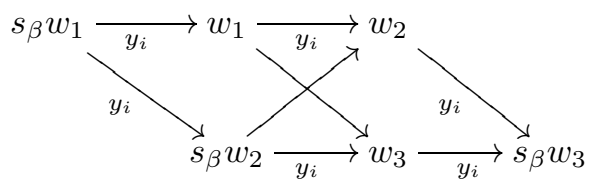

We have by 1)

$$
A_{i}\left(s_{\beta} w_{1}, w_{1}, s_{\beta} w_{2}, w_{2}\right)=A_{i}\left(s_{\beta} w_{2}, w_{2}, w_{3}, s_{\beta} w_{3}\right)=-1
$$

and by 2)

$$
A_{i}\left(s_{\beta} w_{1}, w_{1}, s_{\beta} w_{2}, w_{3}\right)=-1
$$

But

$$
\begin{aligned}
& A_{i}\left(w_{1}, w_{2}, w_{3}, s_{\beta} w_{3}\right) \\
& =A_{i}\left(s_{\beta} w_{1}, w_{1}, s_{\beta} w_{2}, w_{2}\right) A_{i}\left(s_{\beta} w_{2}, w_{2}, w_{3}, s_{\beta} w_{3}\right) A_{i}\left(s_{\beta} w_{1}, s_{\beta} w_{2}, w_{1}, w_{3}\right) .
\end{aligned}
$$

Hence the assertion follows.

4) $w_{2} \neq s_{\beta} w_{1}, w_{4} \neq s_{\beta} w_{2}$. We see as in [BGG, p. 57, c)] that $w_{4} \neq s_{\beta} w_{2}, s_{\beta} w_{3}$, and hence as in [BGG, p. 56,1)] we find that $\left(s_{\beta} w_{1}, s_{\beta} w_{2}, s_{\beta} w_{3}, s_{\beta} w_{4}\right)$ is also a $y_{i}$-twisted square. Hence a) $w_{i}^{-1}(\beta) \in \Delta_{+}^{r e}$ for all $i$ or b) $w_{i}^{-1}(\beta) \in \Delta_{-}^{r e}$ for all $i$.

a) The case $w_{i}^{-1}(\beta) \in \Delta_{+}^{r e}$ for all $i$ : By the definition I) we have the commutative diagram

$$
\begin{array}{cc}
M^{y_{i-1}}\left(w_{1} \circ \lambda\right) \stackrel{\epsilon_{w_{4}, w_{a}}^{i-1} \epsilon_{w_{a}, w_{1}}^{i-1} \varphi_{w_{4}, w_{1}}^{\lambda, y_{i-1}}}{\longrightarrow} & M^{y_{i-1}}\left(w_{4} \circ \lambda\right) \\
\phi_{w_{1}}^{y_{i-1}} \downarrow & \downarrow \phi_{w_{4}}^{y_{i-1}} \\
M^{y}\left(w_{1} \circ \lambda\right) \stackrel{\epsilon_{w_{4}, w_{a}}^{i} \epsilon_{w_{a}, w_{1}}^{i} \varphi_{w_{4}, w_{1}}^{\lambda, y_{i}}}{\stackrel{y_{2}}{i}} & M^{y}\left(w_{4} \circ \lambda\right)
\end{array}
$$

for $a=2,3$. Since $\epsilon_{w_{4}, w_{2}}^{i-1} \epsilon_{w_{2}, w_{1}}^{i-1}=-\epsilon_{w_{4}, w_{3}}^{i-1} \epsilon_{w_{3}, w_{1}}^{i-1}$ by the induction hypothesis the commutativity of the above diagram implies that $\epsilon_{w_{4}, w_{2}}^{i} \epsilon_{w_{2}, w_{1}}^{i}=-\epsilon_{w_{4}, w_{3}}^{i} \epsilon_{w_{3}, w_{1}}^{i}$ by Proposition 6.8 (ii).

b) The case that $w_{i}^{-1}(\beta) \in \Delta_{-}^{r e}$ for all $i$ : We have that $\left(s_{\beta} w_{1}, w_{1}, s_{\beta} w_{2}, w_{2}\right)$, $\left(s_{\beta} w_{1}, w_{1}, s_{\beta} w_{3}, w_{3}\right),\left(s_{\beta} w_{1}, s_{\beta} w_{2}, s_{\beta} w_{3}, s_{\beta} w_{4}\right),\left(s_{\beta} w_{2}, w_{2}, s_{\beta} w_{4}, w_{4}\right)$ and $\left(s_{\beta} w_{3}, w_{3}\right.$, $\left.s_{\beta} w_{4}, w_{4}\right)$ are all $y_{i}$-twisted squares. Hence the assertion follows from the equality

$$
\begin{aligned}
& A_{i}\left(w_{1}, w_{2}, w_{3}, w_{4}\right) A_{i}\left(s_{\beta} w_{1}, s_{\beta} w_{2}, w_{1}, w_{2}\right) A_{i}\left(s_{\beta} w_{1}, w_{1}, s_{\beta} w_{3}, w_{3}\right) \\
& \quad=A_{i}\left(s_{\beta} w_{1}, s_{\beta} w_{2}, s_{\beta} w_{3}, s_{\beta} w_{4}\right) A_{i}\left(s_{\beta} w_{2}, w_{2}, s_{\beta} w_{4}, w_{4}\right) A_{i}\left(s_{\beta} w_{3}, s_{\beta} w_{4}, w_{3}, w_{4}\right) .
\end{aligned}
$$

Let $k$ be an admissible number, $\lambda \in P r_{k}$. Let $y \in \mathcal{W}(\lambda),\left\{y_{i}\right\},\left\{\phi_{w}^{y_{i}}\right\},\left\{\epsilon_{w_{2}, w_{1}}^{i}\right\}$ be as in Proposition 6.7. Because $\left\{\epsilon_{w_{2}, w_{1}}^{i}\right\}$ satisfies the condition in Theorem 6.6 there 
is a corresponding twisted BGG resolution $\mathcal{B}_{\bullet}^{y_{i}}(\lambda)$ of $L(\lambda)$ for $i=0,1, \ldots, l=\ell(y)$. Define

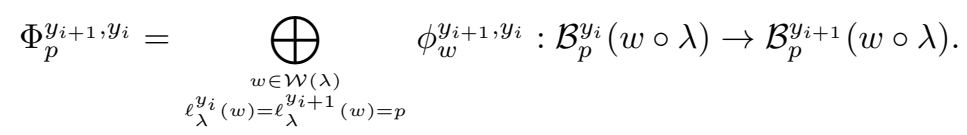

Proposition 6.9. In the above setting $\Phi_{\bullet}^{y_{i+1}, y_{i}}$ gives a quasi-isomorphism $\mathcal{B}_{\bullet}^{y_{i}}(\lambda) \sim$ $\mathcal{B}_{\bullet}^{y_{i+1}}(\lambda)$ of complexes for each $i=0,1, \ldots, l-1$.

Lemma 6.10. Let $\lambda \in \mathfrak{h}^{*}, y, y_{i}$ be as in Proposition 6.7, $w_{1}, w_{2} \in \mathcal{W}(\lambda)$.

(i) Suppose that $w_{1} \triangleright_{\lambda, y_{i}} w_{2}, \ell^{y_{i}}\left(w_{1}\right)=\ell^{y_{i+1}}\left(w_{1}\right)$. Then $w_{1} \triangleright_{\lambda, y_{i+1}} w_{2}$.

(ii) Suppose that $w_{1} \triangleright_{\lambda, y_{i}} w_{2}, \ell^{y_{i}}\left(w_{2}\right)=\ell^{y_{i+1}}\left(w_{2}\right)$. Then either of the following two holds.

(a) $w_{2}=s_{\beta} w_{1}$ and $w_{2} \triangleright_{\lambda, y_{i+1}} w_{1}$.

(b) $w_{1} \triangleright_{\lambda, y_{i+1}} w_{2}$.

Proof. (1) By assumption $s_{\beta} w_{1} \triangleright_{\lambda, y_{i}} w_{2}$. Therefore $\left(s_{\beta} w_{1}, w_{1}, s_{\beta} w_{2}, w_{2}\right)$ is a $y_{i^{-}}$ twisted square. (2) Similarly, if $w_{2} \neq s_{\beta} w_{1}$, then $\left(s_{\beta} w_{1}, w_{1}, s_{\beta} w_{2}, w_{2}\right) y_{i}$-twisted square. The $w_{2} \neq s_{\beta} w_{1}$ case is obvious.

Proof of Proposition 6.9. The fact that $\Phi_{\bullet}^{y_{i}}$ defines a homomorphism of complexes follows from the commutativity of (42), Proposition 6.8 (iii), and Lemma 6.10, Since both complexes are quasi-isomorphic to $L(\lambda)$, to show that it defines a quasiisomorphism it suffices to check that it defines a non-trivial homomorphism between the corresponding homology spaces. This follows from the fact that $\phi_{1}^{y_{i}}: M^{y_{i}}(\lambda) \rightarrow$ $M^{y_{i+1}}(\lambda)$ sends the highest weight vector of $M^{y_{i}}(\lambda)$ to the highest weight vector of $M^{y_{i+1}}(\lambda)$.

\subsection{Two-sided BGG resolutions of $G$-integrable admissible representa-} tions. For $\lambda \in \operatorname{Pr}_{k}$ and $i \in \mathbb{Z}$ set

$$
\mathcal{W}^{i}(\lambda)=\left\{w \in \mathcal{W}(\lambda) ; \ell_{\lambda}^{\frac{\infty}{2}}(w)=i\right\}
$$

We note that

$$
\sharp \mathcal{W}^{i}(\lambda)= \begin{cases}1 & \text { if } \stackrel{\circ}{\mathfrak{g}}=\mathfrak{s l}_{2}, \\ \infty & \text { else. }\end{cases}
$$

Theorem 6.11. Let $k$ be an admissible number, $\lambda \in \operatorname{Pr}_{k}^{+}$:

(i) The space $\operatorname{Hom}_{\mathfrak{g}}\left(W(w \circ \lambda), W\left(w^{\prime} \circ \lambda\right)\right)$ is one-dimensional for $w, w^{\prime} \in \mathcal{W}(\lambda)$ such that $w \triangleright_{\lambda, \frac{\infty}{2}} w^{\prime}$.

(ii) There exists a complex

$$
C^{\bullet}(\lambda): \cdots \rightarrow C^{-2}(\lambda) \stackrel{d_{-2}}{\rightarrow} C^{-1}(\lambda) \stackrel{d_{-1}}{\rightarrow} C^{0}(\lambda) \stackrel{d_{0}}{\rightarrow} C^{1}(\lambda) \stackrel{d_{1}}{\rightarrow} C^{2}(\lambda) \stackrel{d_{2}}{\rightarrow} \cdots
$$

in the category $\mathcal{O}$ of the form

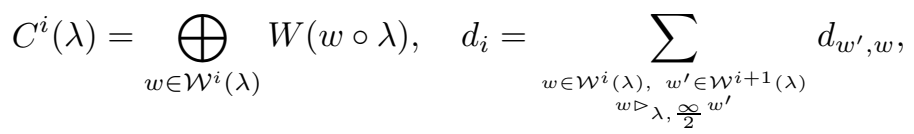

where $d_{w^{\prime}, w}$ is a non-trivial $\mathfrak{g}$-homomorphism $W(w \circ \lambda) \rightarrow W\left(w^{\prime} \circ \lambda\right)$, such that

$$
H^{i}\left(C^{\bullet}(\lambda)\right) \cong \begin{cases}L(\lambda) & \text { for } i=0 \\ 0 & \text { for } i \neq 0\end{cases}
$$


Proof. (ii) Let $q$ be the denominator of $k$ and set $M=q \stackrel{\circ}{Q^{\vee}}$ if $\left(r^{\vee}, q\right)=1$ and $M=q \stackrel{\circ}{Q}$ if $\left(r^{\vee}, q\right)=r^{\vee}$, so that $\mathcal{W}(\lambda)=\stackrel{\circ}{\mathcal{W}} \ltimes t_{M}$. Let $\gamma_{1}, \gamma_{2}, \ldots$, be a sequence in $\stackrel{\circ}{P_{+}^{\vee}} \cap M$ such that $\gamma_{i}-\gamma \in \stackrel{\circ}{P_{+}^{\vee}} \cap M, \lim _{i \rightarrow \infty} \alpha\left(\gamma_{i}\right)=\infty$ for all $\alpha \in \stackrel{\circ}{\Delta}_{+}$.

By Proposition 6.9 there is an inductive system $\left\{\mathcal{B}_{\bullet}^{-\gamma_{i}}(\lambda)\right\}$ of twisted BGG resolutions. Let $\mathcal{B}_{-\gamma_{i}}^{\bullet}(\lambda)$ be the complex $\mathcal{B}_{\bullet}^{-\gamma_{i}}(\lambda)$ with the opposite homological grading. Thus it is a complex

$$
B_{-\gamma_{i}}^{\bullet}(\lambda) \cdots \stackrel{d_{-2}}{\rightarrow} \mathcal{B}_{\gamma_{i}}^{-1}(\lambda) \stackrel{d_{-1}}{\rightarrow} \mathcal{B}_{-\gamma_{i}}^{0}(\lambda) \stackrel{d_{0}}{\rightarrow} B_{\gamma_{i}}^{1}(\lambda) \stackrel{d_{f}}{\rightarrow} \cdots
$$

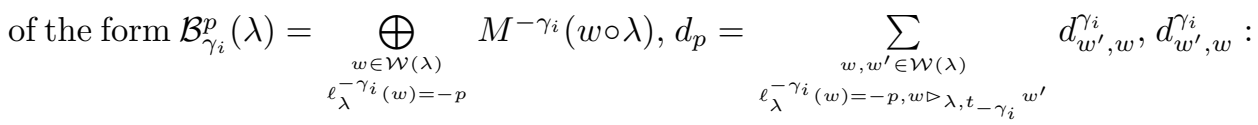
$M^{-\gamma_{i}}(w \circ \lambda) \rightarrow M^{-\gamma_{i}}\left(w^{\prime} \circ \lambda\right)$ such that

$$
H^{p}\left(B_{-\gamma_{i}}^{\bullet}(\lambda)\right)= \begin{cases}L(\lambda) & \text { if } p=0 \\ 0 & \text { otherwise. }\end{cases}
$$

Let $\left(C^{\bullet}(\lambda), d_{\bullet}\right)$ be the complex obtained as the inductive limit of complex $\mathcal{B}_{-\gamma_{i}}^{\bullet}(\lambda)$. By Lemma 3.2 Proposition 4.3 and Proposition 6.9 we have

$$
\begin{aligned}
& C^{p}(\lambda)=\bigoplus_{w \in \mathcal{W}^{p}(\lambda)} \lim _{\vec{i}} M^{-\gamma_{i}}(w \circ \lambda)=\bigoplus_{w \in \mathcal{W}^{p}(\lambda)} W(w \circ \lambda) \quad \text { for } p \in \mathbb{Z}, \\
& H^{p}\left(C^{\bullet}(\lambda)\right)=\lim _{\vec{i}} H^{p}\left(B_{-\gamma_{i}}^{\bullet}(\lambda)\right)= \begin{cases}L(\lambda) & \text { if } p=0, \\
0 & \text { otherwise },\end{cases}
\end{aligned}
$$

and the differential $d_{p}: C^{p}(\lambda) \rightarrow C^{p+1}(\lambda)$ has the form

$$
d_{p}=\sum_{\substack{w \in \mathcal{W}^{p}(\lambda), w^{\prime} \in \mathcal{W}^{p+1} \\ w \triangleright \lambda, \frac{\infty}{2} w^{\prime}}} d_{w^{\prime}, w}
$$

where $d_{w^{\prime}, w}: W(w \circ \lambda) \rightarrow W\left(w^{\prime} \circ \lambda\right)$ is induced by the homomorphisms $d_{w^{\prime}, w}^{-\gamma_{i}}$ : $M^{-\gamma_{i}}(w \circ \lambda) \rightarrow M^{-\gamma_{i}}\left(w^{\prime} \circ \lambda\right)$ with $i=1,2, \ldots$ To complete the proof of (ii) it remains to show that the map $d_{w^{\prime}, w}$ is nonzero for $w \triangleright_{\lambda, \frac{\infty}{2}} w^{\prime}$.

Let $w^{\prime}, w \in \mathcal{W}(\lambda)$ such that $w \triangleright_{\lambda, \frac{\infty}{2}} w^{\prime}$. We have the commutative diagram

$$
\begin{aligned}
& M^{-\gamma_{i}}\left(w^{\prime} \circ \lambda\right) \stackrel{d_{w, w^{\prime}}^{-\gamma_{i}}}{\longrightarrow} M^{-\gamma_{i}}(w \circ \lambda) \\
& \downarrow \phi_{-\gamma_{i}}^{w^{\prime} \circ \lambda} \quad \downarrow \phi_{-\gamma_{i}}^{w \circ \lambda} \\
& W\left(w^{\prime} \circ \lambda\right) \stackrel{d_{w, w^{\prime}}}{\longrightarrow} W(w \circ \lambda)
\end{aligned}
$$

for all $i$. By applying the functor $G_{-\gamma_{i}}$ we obtain the commutative diagram

$$
\begin{array}{cr}
M\left(t_{\gamma_{i}} w^{\prime} \circ \lambda\right) \stackrel{G_{-\gamma_{i}}\left(d_{w, w^{\prime}}^{-\gamma_{i}}\right)}{\longrightarrow} & M\left(t_{\gamma_{i}} w \circ \lambda\right) \\
\downarrow G_{-\gamma_{i}}\left(\phi_{-\gamma_{i}}^{w^{\prime} \circ \lambda}\right) & \downarrow G_{-\gamma_{i}}\left(\phi_{-\gamma_{i}}^{w^{\prime} \circ \lambda}\right) \\
W\left(t_{\gamma_{i}} w^{\prime} \circ \lambda\right) \stackrel{G_{-\gamma_{i}}\left(d_{w, w^{\prime}}\right)}{\longrightarrow} & W\left(t_{\gamma_{i}} w \circ \lambda\right) .
\end{array}
$$


By Corollary $4.5 d_{w, w^{\prime}} \neq 0$ if and only if $G_{-\gamma_{i}}\left(d_{w, w^{\prime}}\right) \neq 0$. Therefore it is sufficient to show that $G_{-\gamma_{i}}\left(\phi_{-\gamma_{i}}^{w^{\prime} \circ \lambda}\right) \circ G_{-\gamma_{i}}\left(d_{w, w^{\prime}}^{-\gamma_{i}}\right): M\left(t_{\gamma_{i}} w^{\prime} \circ \lambda\right) \rightarrow W\left(t_{\gamma_{i}} w \circ \lambda\right)$ is non-zero for a sufficiently large $i$.

Write $w^{\prime}=s_{\alpha} w$ with $\alpha \in \Delta^{r e}, \bar{\alpha} \in \stackrel{\circ}{\Delta}_{-}$. (This is possible because $s_{\alpha}=s_{-\alpha}$.) Then, for a sufficiently large $i, \beta:=t_{\gamma_{i}}(\alpha) \in \Delta_{+}^{r e}$ and $t_{\gamma_{i}} s_{\alpha} w=s_{\beta} t_{\gamma_{i}} w \rightarrow t_{\gamma_{i}} w$. The determinant formula [Fre1, Proposition 2 (2)] shows that the image of the highest weight vector of $M\left(t_{\gamma_{i}} w^{\prime} \circ \lambda\right)=M\left(s_{\beta} t_{\gamma_{i}} w \circ \lambda\right)$ in $M\left(t_{\gamma_{i}} w \circ \lambda\right)$ is not in the kernel of the map $G_{\gamma_{i}}\left(\phi_{\gamma_{i}}^{w^{\prime}, \lambda}\right) ; M\left(t_{\gamma_{i}} w \circ \lambda\right) \rightarrow W\left(t_{\gamma_{i}} w \circ \lambda\right)$. Therefore $G_{\gamma_{i}}\left(\phi_{\gamma_{i}}^{w^{\prime}, \lambda}\right) \circ G_{\gamma_{i}}\left(d_{w, w^{\prime}}^{\gamma_{i}}\right)$ is non-zero, and hence so is $d_{w, w^{\prime}}$.

Finally, we shall prove (i). Note that

$$
\operatorname{Hom}_{\mathfrak{g}}\left(W\left(w^{\prime} \circ \lambda\right), W(w \circ \lambda)\right)=\lim _{i} \operatorname{Hom}_{\mathfrak{g}}\left(M^{-\gamma_{i}}\left(w^{\prime} \circ \lambda\right), W(w \circ \lambda)\right)
$$

and that $\operatorname{Hom}_{\mathfrak{g}}\left(M^{-\gamma_{i}}\left(w^{\prime} \circ \lambda\right), W(w \circ \lambda)\right)$ is at most one-dimensional by the Jantzen sum formula since $w^{\prime} \triangleright_{\lambda} w$. It follows from (the proof of) (ii) that $\operatorname{Hom}_{\mathfrak{g}}\left(W\left(w^{\prime} \circ\right.\right.$ $\lambda), W(w \circ \lambda))$ is spanned by $d_{w, w^{\prime}}$. This completes the proof.

Remark 6.12. By Theorem 6.11 (i) the resolution in Theorem 6.11 (ii) may be described in terms of screening operators as in $[\mathrm{BF}]$ provided that the existence of corresponding cycles is established; see e.g. [TK].

The following assertion is an immediate consequence of Theorem 6.11 which generalizes [FF2, Theorem 4.1].

Theorem 6.13. Let $k$ be an admissible number, $\lambda \in P r_{k}^{+}, p \in \mathbb{Z}$. We have

$$
\begin{aligned}
& H^{\frac{\infty}{2}+p}(\mathfrak{a}, L(\lambda))=\bigoplus_{w \in \mathcal{W}^{p}(\lambda)} \mathbb{C}_{w \circ \lambda} \quad \text { as } \mathfrak{h} \text {-modules, } \\
& H^{\frac{\infty}{2}+p}(L \mathfrak{n}, L(\lambda))=\bigoplus_{w \in \mathcal{W}^{p}(\lambda)} \pi_{w \circ \lambda+h^{\vee} \Lambda_{0}} \quad \text { as } \mathcal{H} \text {-modules. }
\end{aligned}
$$

6.6. A description of vacuum admissible representation. Let $V^{k}(\mathfrak{g})$ be the universal affine vertex algebra associated with $\stackrel{\circ}{\mathfrak{g}}$ at level $k$ :

$$
V^{k}(\stackrel{\circ}{\mathfrak{g}})=U(\mathfrak{g}) \otimes_{U(\stackrel{\circ}{\mathfrak{g}}[t] \oplus \mathbb{C} K)} \mathbb{C}_{k},
$$

where $\mathbb{C}_{k}$ is the one-dimensional representations of $\stackrel{\circ}{\mathfrak{g}}[t] \oplus \mathbb{C} K$ on which $\stackrel{\circ}{\mathfrak{g}}[t]$ acts trivially and $K$ acts as the multiplication by $k$. By [Fre2, Proposition 5.2] we have an injective homomorphism of vertex algebras

$$
V^{k}(\mathfrak{g}) \hookrightarrow W\left(k \Lambda_{0}\right)
$$

for all $k \in \mathbb{C}$. Hence $V^{k}(\mathfrak{g})$ may be regarded as a vertex subalgebra of $W\left(k \Lambda_{0}\right)$.

Note that $L\left(k \Lambda_{0}\right)$ is the unique simple quotient of $V^{k}(\stackrel{\circ}{\mathfrak{g}})$.

Proposition 6.14. Let $k$ be an admissible number, $\Psi: W\left(\dot{s}_{0} \circ k \Lambda_{0}\right) \rightarrow W\left(k \Lambda_{0}\right)$ a non-zero $\mathfrak{g}$-homomorphism, which exists uniquely up to a nonzero constant multiplication by Theorem 6.11(i). Then the image of the highest weight vector of $W\left(\dot{s}_{0} \circ k \Lambda_{0}\right)$ generates the maximal submodule of $V^{k}(\mathfrak{g}) \subset W\left(k \Lambda_{0}\right)$. 
Proof. By [KW1] the maximal submodule of $V^{k}(\mathfrak{g})$ is generated by a singular vector $v$ of weight $\dot{s}_{0} \circ k \Lambda_{0}$. Consider the two-sided resolution $C \cdot\left(k \Lambda_{0}\right)$ of $L\left(k \Lambda_{0}\right)$ in Theorem 6.11 (ii). Because it is a resolution of $L\left(k \Lambda_{0}\right)$ and $V^{k}(\mathfrak{g}) \subset W\left(k \lambda_{0}\right)$, the vector $v$ must be in the image of $d_{1, w}: W\left(w \circ k \Lambda_{0}\right) \rightarrow W\left(k \lambda_{0}\right)$ for some $w \in \mathcal{W}^{-1}\left(k \Lambda_{0}\right)$. Since the weight $w \circ k \Lambda_{0}$ is strictly smaller than $\dot{s}_{0} \circ k \Lambda_{0}$ for $w \in \mathcal{W}^{-1}\left(k \Lambda_{0}\right) \backslash\left\{\dot{s}_{0}\right\}$, the only possibility is that $v$ is the image of the highest weight vector of $W\left(\dot{s}_{0} \circ k \Lambda_{0}\right)$.

\subsection{Two-sided BGG resolutions of more general admissible representa-}

tions. Let $\lambda \in \operatorname{Pr}_{k, y}$ with $y=\bar{y} t_{\eta}, \bar{y} \in \stackrel{\circ}{\mathcal{W}}, \eta \in \stackrel{\circ}{Q^{\vee}}$. Then there exists $\lambda_{1} \in \operatorname{Pr}_{k}^{+}$ such that $\lambda=y \circ \lambda_{1}$. Since $y\left(\Delta\left(\lambda_{1}\right)_{+}\right) \subset \Delta_{+}^{r e}, T_{y}: \mathcal{O}_{\left[\lambda_{1}\right]}^{\mathfrak{g}} \rightarrow \mathcal{O}_{[\lambda]}^{\mathfrak{g}}$ is exact,

$$
\begin{aligned}
& T_{y} L\left(\lambda_{1}\right) \cong L(\lambda), \\
& T_{y} W\left(w \circ \lambda_{1}\right) \cong T_{y} \lim _{\vec{i}} M^{-\gamma_{i}}\left(w \circ \lambda_{1}\right) \cong \underset{\underset{i}{\vec{i}}}{\lim _{y} T_{y} M^{-\gamma_{i}}\left(w \circ \lambda_{1}\right)} \\
& \cong \lim _{\vec{i}} M^{-y\left(\gamma_{i}\right)}\left(y w y^{-1} \circ \lambda\right) \cong W^{\bar{y}}\left(y w y^{-1} \circ \lambda\right)
\end{aligned}
$$

for $w \in \mathcal{W}\left(\lambda_{1}\right)=y^{-1} \mathcal{W}(\lambda) y$ by Proposition 4.14, Lemmas 5.5 and 5.7, where $\left(\gamma_{1}, \gamma_{2}, \ldots,\right)$ is a sequence as in proof of Theorem 6.11. Therefore the following assertion follows immediately from Theorem 6.6.

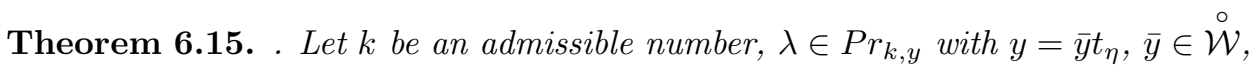
$\eta \in \stackrel{\circ}{P^{\vee}}$. Then there exists a complex

$$
C^{\bullet}(\lambda): \cdots \stackrel{d_{-3}}{\rightarrow} C^{-2}(\lambda) \stackrel{d_{-2}}{\rightarrow} C^{-1}(\lambda) \stackrel{d_{-1}}{\rightarrow} C^{0}(\lambda) \stackrel{d_{0}}{\rightarrow} C^{1}(\lambda) \stackrel{d_{1}}{\rightarrow} C^{2}(\lambda) \stackrel{d_{2}}{\rightarrow} \cdots
$$

in the category $\mathcal{O}$ of the form

$$
C^{i}=\bigoplus_{w \in \mathcal{W}^{i}(\lambda)} W^{\bar{y}}(w \circ \lambda), d_{i}=\sum_{\substack{w \in \mathcal{W}^{i}(\lambda), w^{\prime} \in \mathcal{W}^{i+1}(\lambda) \\ w \triangleright_{\lambda, \frac{\infty}{2}} w^{\prime}}} d_{w^{\prime}, w} .
$$

such that

$$
H^{i}\left(C^{\bullet}(\lambda)\right) \cong \begin{cases}L(\lambda) & \text { for } i=0 \\ 0 & \text { for } i \neq 0\end{cases}
$$

Remark 6.16. If $\lambda \in \operatorname{Pr}_{k, y}$ and $\bar{y}=1$ (that is, $\left.y \in \stackrel{\circ \vee}{P}\right)$, then $W^{\bar{y}}(w \circ \lambda)=W(w \circ \lambda)$. Hence the above is the resolution of $L(\lambda)$ in terms of (non-twisted) Wakimoto modules as conjectured in [FKW].

\section{SEMi-INFinite RESTRICTION AND INDUCTION}

7.1. Feigin-Frenkel parabolic induction. Let $\stackrel{\circ}{\mathfrak{p}}$ be a parabolic subalgebra of $\stackrel{\circ}{\mathfrak{g}}$ containing $\stackrel{\circ}{\mathfrak{b}}_{-}$, and let $\stackrel{\circ}{\mathfrak{p}}=\stackrel{\circ}{\mathfrak{l}} \oplus \stackrel{\circ}{\mathfrak{m}}_{-}$be the direct sum decomposition of $\stackrel{\circ}{\mathfrak{p}}$ with the Levi subalgebra $\stackrel{\circ}{\mathfrak{l}}$ containing $\stackrel{\circ}{\mathfrak{h}}$ and the nilpotent radical $\stackrel{\circ}{\mathfrak{m}}$.. Denote by $\stackrel{\circ}{\mathfrak{m}} \subset \stackrel{\circ}{\mathfrak{n}}$ the opposite algebra of $\stackrel{\circ}{\mathfrak{m}}$, so that $\stackrel{\circ}{\mathfrak{g}}=\stackrel{\circ}{\mathfrak{p}} \oplus \stackrel{\circ}{\mathfrak{m}}$. Let

$$
\stackrel{\circ}{\mathfrak{l}}=\stackrel{\mathfrak{l}}{0}_{0} \oplus \bigoplus_{i=1}^{s} \stackrel{\circ}{\mathfrak{l}}_{i}
$$


be the decomposition of $\stackrel{0}{\mathfrak{l}}$ into direct sum of simple Lie subalgebras $\stackrel{\circ}{\mathfrak{l}}_{i}, i=1, \ldots, s$, and its center $\stackrel{\circ}{\mathfrak{l}}_{0}$ of $\stackrel{\circ}{\mathfrak{l}}$. Let $\stackrel{\circ}{\mathfrak{h}}_{i}=\mathfrak{i} \cap \stackrel{\circ}{\mathfrak{h}}$, the Cartan subalgebra of $\stackrel{\circ}{\mathfrak{l}}_{i}$, and denote by $\stackrel{\circ}{\Delta}_{i} \subset \stackrel{\circ}{\Delta}$ the subroot system of $\stackrel{\circ}{\mathfrak{g}}$ corresponding to $\stackrel{\circ}{\mathfrak{l}}_{i}, \stackrel{\circ}{\Pi}_{i}=\stackrel{\circ}{\Pi} \cap \stackrel{\circ}{\Delta}_{i}$. Let $h_{i}^{\vee}$ be the dual Coxeter number of $\stackrel{\circ}{\mathfrak{l}}_{i}$ (with a convention $h_{0}^{\vee}=0$ ), $\theta_{i}$ the highest root of $\stackrel{\circ}{\Delta}_{i}$, $\theta_{i, s}$ the highest short roof of $\stackrel{\circ}{\Delta}_{i}$.

Let $\mathfrak{l}_{i}=\stackrel{\circ}{\mathfrak{l}}_{i}\left[t, t^{-1}\right] \oplus \mathbb{C} K \subset \mathfrak{g}$ for $i=0,1, \ldots, s$. Set

$$
K_{i}=\frac{2}{\left(\theta_{i} \mid \theta_{i}\right)} K
$$

and we consider $K_{i}$ as an element of $\mathfrak{l}_{i}$. Thus,

$$
\mathfrak{l}_{i}=\stackrel{\circ}{\mathfrak{l}}_{i}\left[t, t^{-1}\right] \oplus \mathbb{C} K_{i},
$$

and $\mathfrak{h}_{i}:=\stackrel{\circ}{\mathfrak{h}}_{i} \oplus \mathbb{C} K_{i}$ is a Cartan subalgebra of $\stackrel{\circ}{\mathfrak{l}}_{i}$.

Define

$$
\mathfrak{l}=\bigoplus_{i=0}^{s} \mathfrak{r}_{i} . \quad \mathfrak{t}=\bigoplus_{i=0}^{s} \mathfrak{h}_{i} .
$$

The grading of $\mathfrak{l}_{i}$ induces the grading of $\mathfrak{l}$.

For $k \in \mathbb{C}$ define $k_{0}, \ldots, k_{s} \in \mathbb{C}$ by

$$
k_{0}=k+h^{\vee}, \quad k_{i}+h_{i}^{\vee}=\frac{2}{\left(\theta_{i} \mid \theta_{i}\right)}\left(k+h^{\vee}\right) \quad \text { for } i=1, \ldots, s .
$$

Lemma 7.1. Let $k$ be an admissible number for $\mathfrak{g}$. Then $k_{i}, i=1, \ldots, s$, is an admissible number for the Kac-Moody algebra $\mathfrak{l}_{i}$.

Let $\mathcal{O}_{\left(k_{0}, \ldots, k_{s}\right)}^{\mathfrak{l}}$ be the full subcategory of $\mathcal{O}^{\mathfrak{l}}$ consisting of objects on which $K_{i}$ acts as the multiplication by $k_{i}, i=0,1, \ldots, s$. Feigin and Frenkel [FF2, 5.2], [Fre2, §6] constructed a functor

$$
\mathrm{F}-i n d_{\mathfrak{l}}^{\mathfrak{g}}: \mathcal{O}_{\left(k_{0}, k_{1}, \ldots, k_{s}\right)}^{\mathfrak{l}} \rightarrow \mathcal{O}_{k}^{\mathfrak{g}}, \quad M \rightarrow \mathrm{F}-\operatorname{ind}_{\mathfrak{l}}^{\mathfrak{g}}(M),
$$

which enjoys the property

$$
\mathrm{F}_{-\operatorname{ind}_{\mathfrak{l}}}^{\mathfrak{g}}(M) \cong U S(L \stackrel{\circ}{\mathfrak{m}}) \otimes_{\mathbb{C}} M
$$

as modules over

$$
L \stackrel{\circ}{\mathfrak{m}}=\stackrel{\circ}{\mathfrak{m}}\left[t, t^{-1}\right] \subset \mathfrak{g},
$$

where $L \stackrel{\circ}{\mathfrak{m}}$ only on the first factor $U S(L \stackrel{\circ}{\mathfrak{m}})$. In particular F-ind $\mathfrak{l}_{\mathfrak{l}}^{\mathfrak{g}}$ is an exact functor.

Denote by $W_{\mathfrak{l}_{i}}\left(\lambda^{(i)}\right)$ the Wakimoto module of the affine Kac-Moody algebra $\mathfrak{l}_{i}$ with highest weight $\lambda^{(i)} \in \mathfrak{h}_{i}{ }^{*}$ and by $L_{\mathfrak{l}}\left(\lambda^{(i)}\right)$ the irreducible highest weight representation of $\mathfrak{l}_{i}$ with highest weight $\lambda^{(i)}$ (with a convention that $W_{\mathfrak{l}_{0}}\left(\lambda^{(0)}\right)$ is the irreducible representation of the Heisenberg algebra $\mathfrak{l}_{0}$ with highest weight $\left.\lambda^{(0)}\right)$. For $\lambda \in \mathfrak{t}^{*}$ let $W_{\mathfrak{l}}(\lambda)$ and $L_{\mathfrak{l}}(\lambda)$ be the Wakimoto module and the irreducible highest weight representation of $\mathfrak{l}$ with highest weight $\lambda$ :

$$
W_{\mathfrak{l}}(\lambda)=\bigotimes_{i=0}^{s} W_{\mathfrak{l}_{i}}\left(\left.\lambda\right|_{\mathfrak{h}_{i}}\right), \quad L_{\mathfrak{l}}(\lambda)=\bigotimes_{i=0}^{s} L_{\mathfrak{l}_{i}}\left(\left.\lambda\right|_{\mathfrak{h}_{i}}\right)
$$


For $\lambda \in \mathfrak{h}^{*}$, define $\lambda_{\mathfrak{l}} \in \mathfrak{t}^{*}$ by

$$
\left.\lambda_{\mathfrak{l}}\right|_{\mathfrak{h}_{i}}=\left.\lambda\right|_{{\stackrel{\circ}{\mathfrak{h}_{i}}}_{i}} \text { and }\left(\lambda_{\mathfrak{l}}+\rho_{i}\right)\left(K_{i}\right)=\frac{2}{\left(\theta_{i} \mid \theta_{i}\right)}(\lambda+\rho)(K)
$$

for $i=0,1, \ldots, s$.

Proposition 7.2 ([FF2]). For $\lambda \in \mathfrak{h}^{*}$ we have F-ind ${ }_{\mathfrak{p}}^{\mathfrak{g}} W_{\mathfrak{l}}\left(\lambda_{\mathfrak{l}}\right) \cong W(\lambda)$.

Proof. By using the Hochschild-Serre spectral sequence for $L \stackrel{\circ}{\mathfrak{m}} \subset \mathfrak{a}$ we see from (50) that

$$
H^{\frac{\infty}{2}+i}\left(\mathfrak{a}, \mathrm{F}-\operatorname{ind}_{\mathfrak{l}}^{\mathfrak{g}} W_{\mathfrak{l}}\left(\lambda_{\mathfrak{l}}\right)\right) \cong \begin{cases}\mathbb{C}_{\lambda} & \text { for } i=0 \\ 0 & \text { otherwise }\end{cases}
$$

Hence the assertion follows from Theorem 4.7

7.2. Semi-infinite restriction functors. Let $M \in \mathcal{O}_{k}^{\mathfrak{g}}$. Then $H^{\frac{\infty}{2}}+p(L \stackrel{\circ}{\mathfrak{m}}, M)$, $p \in \mathbb{Z}$, is naturally an l-module on which $K_{i}$ acts as the multiplication by $k_{i}$; see e.g. [HT, Proposition 2.3]. Hence

$$
\mathrm{S}_{-\mathrm{res}_{\mathfrak{l}}^{\mathfrak{g}}}^{\mathfrak{s}}:=H^{\frac{\infty}{2}+0}(L \stackrel{\circ}{\mathfrak{m}}, ?)
$$

defines a functor $\mathcal{O}_{k}^{\mathfrak{g}} \rightarrow \mathcal{O}_{\left(k_{0}, k_{1}, \ldots, k_{s}\right)}^{\mathfrak{l}}$. We refer to S-res $\mathfrak{l}_{\mathfrak{l}}^{\mathfrak{g}}$ as the semi-infinite restriction functor.

The following assertion follows from Proposition 7.2 .

Proposition 7.3. For $\lambda \in \mathfrak{h}^{*}$ we have $H^{\frac{\infty}{2}+i}(L \stackrel{\circ}{\mathfrak{m}}, W(\lambda))=0$ for $i \neq 0$ and

$$
\text { S-res: } \mathfrak{l} W(\lambda) \cong W_{\mathfrak{l}}\left(\lambda_{\mathfrak{l}}\right) \text {. }
$$

7.3. Decomposition of integral Weyl groups. Let $k$ be an admissible number with denominator $q, \lambda \in P r_{k}^{+}$. Let $\stackrel{\circ}{\mathcal{W}}_{S_{i}}$ be the parabolic subgroup of $\stackrel{\circ}{\mathcal{W}}$ corresponding to $\stackrel{\circ}{i}_{i}, \stackrel{\circ}{\mathcal{W}}_{S}=\stackrel{\circ}{\mathcal{W}}_{S_{1}} \times \stackrel{\circ}{\mathcal{W}}_{S_{2}} \times \cdots \times \stackrel{\circ}{\mathcal{W}}_{S_{s}}$. Define $\dot{\alpha}_{0}^{(i)} \in \Delta(\lambda), i=1, \ldots, s$, by

$$
\begin{gathered}
\dot{\alpha}_{0}^{(i)}=-\theta_{i}+q \delta \quad \text { if }\left(r^{\vee}, q\right)=1, \\
\text { and }\left(\dot{\alpha}_{0}^{(i)}\right)^{\vee}=-\theta_{i, s}^{\vee}+q \delta \quad \text { if }\left(r^{\vee}, q\right)=r^{\vee} .
\end{gathered}
$$

Set $\dot{s}_{0}^{(i)}=s_{\dot{\alpha}_{0}^{(i)}}$.

Let $\mathcal{W}(\lambda)_{S_{i}}$ be the subgroup of $\mathcal{W}(\lambda)$ generated by $\stackrel{\circ}{\mathcal{W}}_{S_{i}}$ and $\dot{s}_{0}^{(i)}$. Then

$$
\mathcal{W}(\lambda)_{S}=\mathcal{W}(\lambda)_{S_{1}} \times \mathcal{W}(\lambda)_{S_{2}} \times \cdots \times \mathcal{W}(\lambda)_{S_{s}}
$$

is the subgroup corresponding to $\stackrel{\circ}{\mathcal{W}}_{S}$ described in 33.4 Let $\mathcal{W}(\lambda)^{S} \subset \mathcal{W}(\lambda)$ be as in Theorem 3.3 so that

$\mathcal{W}(\lambda)=\mathcal{W}(\lambda)_{S} \times \mathcal{W}(\lambda)^{S}, \quad \ell_{\lambda}^{\frac{\infty}{2}}(u v)=\ell_{\lambda}^{\frac{\infty}{2}}(u)+\ell_{\lambda}^{\frac{\infty}{2}}(v)$ for $u \in \mathcal{W}(\lambda)_{S}, v \in \mathcal{W}(\lambda)^{S}$.

Let $w, w^{\prime} \in \mathcal{W}(\lambda)_{S_{i}} \subset \mathcal{W}(\lambda)$ such that $w \triangleright_{\lambda, \frac{\infty}{2}} w^{\prime}$. Then $w \circ_{\mathfrak{l}_{i}} \lambda_{\mathfrak{l}}^{(i)}=(w \circ \lambda)_{\mathfrak{l}}^{(i)}$, where $\circ_{\mathfrak{l}_{i}}$ is the dot action of $\mathcal{W}(\lambda)_{S_{i}}$ on $\mathfrak{h}_{i}^{*}$ and $\lambda_{\mathfrak{l}_{i}}^{(i)}=\left.\lambda_{\mathfrak{l}}\right|_{\mathfrak{h}_{i}}$. 
Proposition 7.4. Let $\lambda \in \operatorname{Pr}_{k}^{+}, w, w^{\prime} \in \mathcal{W}(\lambda)_{S_{i}}$ with $i \in\{1,2, \ldots, s\}$ such that $w \triangleright_{\lambda, \frac{\infty}{2}} w^{\prime}$. Then the correspondence $\Phi \mapsto \mathrm{F}$-ind $d_{\mathfrak{l}}^{\mathfrak{g}}(\Phi)$ defines a linear isomorphism

$$
\operatorname{Hom}_{\mathfrak{l}}\left(W_{\mathfrak{l}}\left((w \circ \lambda)_{\mathfrak{l}}\right), W_{\mathfrak{l}}\left(\left(w^{\prime} \circ \lambda\right) \mathfrak{l}\right)\right) \stackrel{\sim}{\rightarrow} \operatorname{Hom}_{\mathfrak{g}}\left(W(w \circ \lambda), W\left(w^{\prime} \circ \lambda\right)\right) .
$$

The inverse map is given by $\Psi \rightarrow \mathrm{S}_{-} \operatorname{res}_{\mathfrak{l}}^{\mathfrak{g}}(\Psi)$.

Proof. By Proposition 4.6 and Theorem 6.11 (i) both $\operatorname{Hom}_{\mathfrak{l}}\left(W_{\mathfrak{l}}\left((w \circ \lambda)_{\mathfrak{l}}\right), W_{\mathfrak{l}}\left(\left(w^{\prime} \circ\right.\right.\right.$ $\left.\left.\lambda)_{\mathfrak{l}}\right)\right)$ and $\operatorname{Hom}_{\mathfrak{g}}\left(W(w \circ \lambda), W\left(w^{\prime} \circ \lambda\right)\right)$ are one-dimensional. The assertion follows

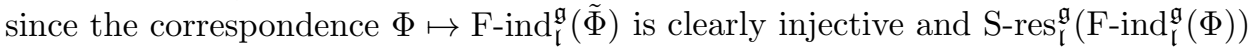
$=\Phi$.

7.4. Semi-infinite restriction of admissible affine vertex algebras. Since it is defined by the semi-infinite cohomology the space $\operatorname{S}_{-} \operatorname{res}_{\mathfrak{l}}^{\mathfrak{g}}\left(V^{k}(\stackrel{\circ}{\mathfrak{g}})\right)$ inherits a vertex algebra structure from $V^{k}(\mathfrak{g})$, and we have a natural vertex algebra homomorphism

$$
\bigotimes_{i=0}^{s} V^{k_{i}}\left(\stackrel{\circ}{\mathfrak{l}}_{i}\right) \rightarrow \operatorname{S-res}_{\mathfrak{l}}^{\mathfrak{g}}\left(V^{k}(\stackrel{\circ}{\mathfrak{g}})\right),
$$

where $V^{k_{i}}\left(\stackrel{\circ}{\mathfrak{l}}_{i}\right)$ denote the universal affine vertex algebra associated with $\stackrel{\circ}{\mathfrak{l}}_{i}$ at level $k_{i}$. By composing with the map $\operatorname{S} \operatorname{res}_{\mathfrak{l}}^{\mathfrak{g}}\left(V^{k}(\stackrel{\circ}{\mathfrak{g}})\right) \rightarrow \operatorname{S} \operatorname{res}_{\mathfrak{l}}^{\mathfrak{g}}\left(L\left(k \Lambda_{0}\right)\right)$ induced by the surjection $V^{k}(\mathfrak{g}) \rightarrow L\left(k \Lambda_{0}\right)$ this gives rise to a vertex algebra homomorphism

$$
\bigotimes_{i=0}^{s} V^{k_{i}}\left(\mathfrak{l}_{i}\right) \rightarrow \operatorname{S-res}_{\mathfrak{l}}^{\mathfrak{g}}\left(L\left(k \Lambda_{0}\right)\right) .
$$

On the other hand, there is a natural surjective homomorphism

$$
\bigotimes_{i=0}^{s} V^{k_{i}}\left(\mathfrak{l}_{i}\right) \rightarrow \bigotimes_{i=0}^{s} L_{\mathfrak{l}_{i}}\left(k_{i} \Lambda_{0}\right)
$$

of vertex algebras, where $L_{\mathfrak{l}_{i}}\left(k_{i} \Lambda_{0}\right)$ is the unique simple quotient of $V^{k_{i}}\left(\mathfrak{l}_{i}\right)$.

Theorem 7.5. Let $k$ be an admissible number. The vertex algebra homomorphism (52) factors through the vertex algebra homomorphism

$$
\bigotimes_{i=0}^{s} L_{\mathfrak{l}_{i}}\left(k_{i} \Lambda_{0}\right) \hookrightarrow \operatorname{S} \operatorname{res}_{\mathfrak{l}}^{\mathfrak{g}}\left(L\left(k \Lambda_{0}\right)\right) .
$$

Proof. Put $\lambda=k \Lambda_{0}$ and let $C^{\bullet}(\lambda)$ be the two-sided BGG resolution of $L\left(k \Lambda_{0}\right)$ in Theorem 6.11. By the vanishing assertion of Proposition 7.3 the semi-infinite cohomology $H^{\frac{\infty}{2}+\bullet}(L \stackrel{\circ}{\mathfrak{m}}, L(\lambda))$ is isomorphic to the cohomology of the complex $\operatorname{S-res}_{\mathfrak{l}}^{\mathfrak{g}}\left(C^{\bullet}(\lambda)\right)$ obtained from $C^{\bullet}(\lambda)$ applying the functor S-res $\mathfrak{l}_{\mathfrak{l}}^{\mathfrak{g}}$. Thus S-res ${ }_{\mathfrak{l}}^{\mathfrak{g}}\left(L\left(k \Lambda_{0}\right)\right)$ is isomorphic to the zeroth cohomology of the complex $\operatorname{S}^{-r_{1}} \mathfrak{l}_{\mathfrak{l}}^{\mathfrak{g}}\left(C^{\bullet}(\lambda)\right)$.

Consider the map $C^{-1}(\lambda) \supset W\left(\dot{s}_{0}^{(i)} \circ \lambda\right) \stackrel{d_{1, \dot{s}_{0}^{(i)}}}{\rightarrow} W(\lambda) \subset C^{0}(\lambda)$ for $i=1, \ldots, s$. By applying the functor S-res ${ }_{\mathfrak{l}}^{\mathfrak{g}}$ this induces a non-zero homomorphism

$$
W_{\mathfrak{l}}\left(\dot{s}_{0}^{(i)} \circ_{\mathfrak{l}_{i}} \lambda_{\mathfrak{l}}\right) \rightarrow W_{\mathfrak{l}}\left(\lambda_{\mathfrak{l}}\right)
$$

by Proposition 17.4, and the image of the highest weight vector of $W_{\mathfrak{l}}\left(\dot{s}_{0}^{(i)} \circ_{\mathfrak{l}_{i}} \lambda_{\mathfrak{l}}\right)$ generates the maximal $\mathfrak{l}_{i}$-submodule of $V^{k_{i}}\left(\mathfrak{l}_{i}\right) \subset W_{\mathfrak{l}}\left(\lambda_{\mathfrak{l}}\right)$ by Proposition 6.14. It follows that the maximal l-submodule of $\bigotimes_{i=0}^{s} V^{k_{i}}\left(\mathfrak{l}_{i}\right) \subset W_{\mathfrak{l}}(\lambda)$ is in the image of $\operatorname{S} \operatorname{res}_{\mathfrak{l}}^{\mathfrak{g}}\left(d_{-1}\right): \operatorname{S}_{-\operatorname{res}_{\mathfrak{l}}}^{\mathfrak{g}}\left(C^{-1}(\lambda)\right) \rightarrow \operatorname{S}_{-\operatorname{res}_{\mathfrak{l}}}^{\mathfrak{g}}\left(C^{0}(\lambda)\right)$. This completes the proof. 
7.5. The case of minimal parabolic subalgebras. Consider the case that $\stackrel{\circ}{\mathfrak{p}}$ is generated by $\stackrel{\circ}{\mathfrak{b}}_{-}$and $e_{i}$ with $i \in \stackrel{\circ}{I}$. Then $\stackrel{\circ}{\mathfrak{l}}=\stackrel{\circ}{\mathfrak{l}}_{0} \oplus \stackrel{\circ}{\mathfrak{l}}_{1}, \stackrel{\circ}{\mathfrak{l}}_{1}=\mathfrak{s l}_{2}^{(i)}$ and $\mathfrak{l}_{1}=\widehat{\mathfrak{s l}}_{2}^{(i)}$.

Theorem 7.6 ( $\stackrel{\circ}{\mathfrak{p}}$ minimal). Let $k$ be an admissible number and let $M$ be a module over the vertex algebra $L\left(k \Lambda_{0}\right)$. Then, for each $p \in \mathbb{Z}, H^{\frac{\infty}{2}}+p(L \stackrel{\circ}{\mathfrak{m}}, M)$ is a direct sum of admissible representations of level $k_{1}$ (see (49)) as $\widehat{\mathfrak{s l}}_{2}^{(i)}$-modules.

Proof. By Theorem [7.5, $L_{\mathfrak{l}_{1}}\left(k_{1} \Lambda_{0}\right)$ is a vertex subalgebra of $\operatorname{S-res}_{\mathfrak{l}}^{\mathfrak{g}}\left(L\left(k \Lambda_{0}\right)\right)=$ $H^{\frac{\infty}{2}+0}\left(L \stackrel{\circ}{\mathfrak{m}}, L\left(k \Lambda_{0}\right)\right)$. If $M$ is a module over $L\left(k \Lambda_{0}\right)$, then $H^{\frac{\infty}{2}}+p(L \stackrel{\circ}{\mathfrak{m}}, M)$ is naturally a module over $\operatorname{S-res}_{\mathfrak{l}}^{\mathfrak{g}}\left(L\left(k \Lambda_{0}\right)\right)$, and therefore, it is a module over $L_{\mathfrak{l}_{1}}\left(k_{1} \Lambda_{0}\right)$. The assertion follows since it is known by [AM] that any module over $L_{\mathfrak{l}_{1}}\left(k_{1} \Lambda_{0}\right)$ in the category $\mathcal{O}^{\mathfrak{l}_{1}}$ must be a direct sum of admissible representations of $\mathfrak{l}_{1} \cong \widehat{\mathfrak{s l}}_{2}$.

The following assertion generalizes $[\mathrm{HT}$, Theorem 3.8] in the case that $\stackrel{\circ}{\mathfrak{p}}$ is minimal.

Theorem 7.7 (’’ minimal). Let $k$ be an admissible number, $\lambda \in \operatorname{Pr}_{k}^{+}$. Then

$$
H^{\frac{\infty}{2}+p}(L \stackrel{\circ}{\mathfrak{m}}, L(\lambda)) \cong \bigoplus_{\substack{w \in \mathcal{W}(\lambda) S \\ \ell^{\frac{\infty}{2}}(w)=p}} L_{\mathfrak{l}}\left((w \circ \lambda)_{\mathfrak{l}}\right)
$$

as $\mathfrak{l}$-modules.

Proof. It is known by $\mathrm{MF}$ (see also $[\mathrm{FM}]$ ) that $L(\lambda)$ with $\lambda \in \operatorname{Pr}_{k}^{+}$is a module over $L\left(k \Lambda_{0}\right)$. Therefore $H^{\frac{\infty}{2}+\bullet}(L \stackrel{\circ}{\mathfrak{m}}, L(\lambda))$ is a direct sum of irreducible admissible representations as $\widehat{\mathfrak{s l}}_{2}^{(i)}$-modules by Theorem [7.6. Hence it is sufficient to determine the subspace $H^{\frac{\infty}{2}+\bullet}(L \stackrel{\circ}{\mathfrak{m}}, L(\lambda))^{\mathfrak{l}_{+}}$of the singular vectors of $H^{\frac{\infty}{2}+\bullet}(L \stackrel{\circ}{\mathfrak{m}}, L(\lambda))$. Clearly, any weight of $H^{\frac{\infty}{2}+\bullet}(L \stackrel{\circ}{\mathfrak{m}}, L(\lambda))^{\mathfrak{l}_{+}}$must be admissible for $\mathfrak{l}_{1}=\widehat{\mathfrak{s l}}_{2}^{(i)}$.

As is remarked in the proof of Proposition 7.5, $H^{\frac{\infty}{2}+\bullet}(L \stackrel{\circ}{\mathfrak{m}}, L(\lambda))$ is the cohomology of the complex S-res ${ }_{\mathfrak{l}}^{\mathfrak{g}}\left(C^{\bullet}(\lambda)\right)$ and we have $\operatorname{S}^{-r e s} \mathfrak{l}_{\mathfrak{l}}^{\mathfrak{g}}\left(C^{p}(\lambda)\right)=\bigoplus_{w \in \mathcal{W}^{p}(\lambda)} W_{\mathfrak{l}}((w \circ$ $\lambda)_{\mathfrak{l}}$ ) by Proposition 7.3 . Now Theorem 3.3 and Lemma 7.1 imply that

$$
\begin{aligned}
& \left\{(w \circ \lambda)_{\mathfrak{l}} ; w \in \mathcal{W}(\lambda),(w \circ \lambda)_{\mathfrak{l}} \text { is an admissible weight for } \widehat{\mathfrak{s l}}_{2}^{(i)}\right\} \\
= & \left\{(w \circ \lambda)_{\mathfrak{l}} ; w \in \mathcal{W}(\lambda),(w \circ \lambda)_{\mathfrak{l}} \text { is a dominant weight for } \widehat{\mathfrak{s l}}_{2}^{(i)}\right\} \\
= & \left\{(w \circ \lambda)_{\mathfrak{l}} ; w \in \mathcal{W}(\lambda)^{S}\right\} .
\end{aligned}
$$

It follows that if a weight $\mu$ of $W_{\mathfrak{l}}\left((w \circ \lambda)_{\mathfrak{l}}\right)$ is admissible for $\widehat{\mathfrak{s l}}_{2}^{(i)}$, then $w \in \mathcal{W}(\lambda)^{S}$ and $\mu=(w \circ \lambda)_{\mathfrak{r}}$. Therefore the image $\left[\left|(w \circ \lambda)_{\mathfrak{r}}\right\rangle\right]$ of the highest weight vector $\left|(w \circ \lambda)_{\mathfrak{l}}\right\rangle$ of $W_{\mathfrak{l}}\left((w \circ \lambda)_{\mathfrak{l}}\right)$ is non-zero in $H^{\frac{\infty}{2}+\bullet}(L \stackrel{\circ}{\mathfrak{m}}, L(\lambda))$ and $\{[|(w \circ \lambda) \mathfrak{l}\rangle] ; w \in$ $\left.\mathcal{W}(\lambda)^{S}\right\}$ forms a basis of $H^{\frac{\infty}{2}+\bullet}(L \stackrel{\circ}{\mathfrak{m}}, L(\lambda))^{\mathfrak{r}_{+}}$. By Theorem 3.3. this completes the proof.

Remark 7.8. In the subsequent paper A6 we prove that for an admissible number $k$ any $L\left(k \Lambda_{0}\right)$-module in the category $\mathcal{O}^{\mathfrak{g}}$ must be a direct sum of admissible representations. Hence it follows from the proof that the assertion of Theorem [7.7 is valid for any parabolic subalgebra of $\stackrel{\circ}{\mathfrak{g}}$. 


\section{ACKNOWLEDGMENTS}

Some part of this work was done while the author was visiting Weizmann Institute, Israel, in May 2011, Emmy Noether Center in Erlangen, Germany in June 2011, Isaac Newton Institute for Mathematical Sciences, UK, in 2011, The University of Manchester, University of Birmingham, The University of Edinburgh, Lancaster University, York University, UK, in November 2011, Academia Sinica, Taiwan, in December 2011. He is grateful to those institutes for their hospitality.

\section{REFERENCES}

[AG] S. Arkhipov and D. Gaitsgory, Differential operators on the loop group via chiral algebras, Int. Math. Res. Not. 4 (2002), 165-210, DOI 10.1155/S1073792802102078. MR.1876958 (2003f:20077)

[AL] H. H. Andersen and N. Lauritzen, Twisted Verma modules, Studies in memory of Issai Schur (Chevaleret/Rehovot, 2000), Progr. Math., vol. 210, Birkhäuser Boston, Boston, MA, 2003, pp. 1-26. MR.1985191(2004d:17005)

[AM] Dražen Adamović and Antun Milas, Vertex operator algebras associated to modular invariant representations for $A_{1}^{(1)}$, Math. Res. Lett. 2 (1995), no. 5, 563-575, DOI 10.4310/MRL.1995.v2.n5.a4. MR1359963 (96m:17047)

[A1] Tomoyuki Arakawa, Vanishing of cohomology associated to quantized Drinfeld-Sokolov reduction, Int. Math. Res. Not. 15 (2004), 730-767, DOI 10.1155/S1073792804132479. MR2040965 (2005e:17033)

[A2] Tomoyuki Arakawa, Representation theory of superconformal algebras and the Kac-RoanWakimoto conjecture, Duke Math. J. 130 (2005), no. 3, 435-478, DOI 10.1215/S00127094-05-13032-0. MR2184567 (2007a:17039)

[A3] Tomoyuki Arakawa, Representation theory of $\mathcal{W}$-algebras, Invent. Math. 169 (2007), no. 2, 219-320, DOI 10.1007/s00222-007-0046-1. MR2318558(2009d:17039)

[A4] Tomoyuki Arakawa, Representation theory of W-algebras, II, Exploring new structures and natural constructions in mathematical physics, Adv. Stud. Pure Math., vol. 61, Math. Soc. Japan, Tokyo, 2011, pp. 51-90. MR2867144

[A5] Tomoyuki Arakawa, Associated varieties of modules over Kac-Moody algebras and $C_{2}$ cofiniteness of $W$-algebras. arXiv:1004.1554[math.QA].

[A6] T. Arakawa, Rationality of admissible affine vertex algebras in the category $\mathcal{O}$, arXiv:1207.4857[math.QA].

[A7] Tomoyuki Arakawa, Rationality of W-algebras; principal nilpotent cases, arXiv:1211.7124[math.QA].

[Ark1] Sergey Arkhipov, A new construction of the semi-infinite BGG resolution. preprint, 1996. math.QA/9605043.

[Ark2] S. M. Arkhipov, Semi-infinite cohomology of associative algebras and bar duality, Internat. Math. Res. Notices 17 (1997), 833-863, DOI 10.1155/S1073792897000548. MR.1474841 (98j:16006)

[AS] Henning Haahr Andersen and Catharina Stroppel, Twisting functors on $\mathcal{O}$, Represent. Theory 7 (2003), 681-699 (electronic), DOI 10.1090/S1088-4165-03-00189-4. MR2032059 (2004k:17010)

[BF] D. Bernard and G. Felder, Fock representations and BRST cohomology in SL(2) current algebra, Comm. Math. Phys. 127 (1990), no. 1, 145-168. MR1036119 (91g:17022)

[BGG] I. N. Bernšteı̌n, I. M. Gel'fand, and S. I. Gel'fand. Differential operators on the base affine space and a study of $\mathfrak{g}$-modules. In Lie groups and their representations (Proc. Summer School, Bolyai János Math. Soc., Budapest, 1971), pages 21-64. Halsted, New York, 1975.

[Fěl] B. L. Fer̆gin, Semi-infinite homology of Lie, Kac-Moody and Virasoro algebras (Russian), Uspekhi Mat. Nauk 39 (1984), no. 2(236), 195-196. MR740035 (85g:17003)

[FF1] B. L. Fergin and E. V. Frenkel, A family of representations of affine Lie algebras (Russian), Uspekhi Mat. Nauk 43 (1988), no. 5(263), 227-228, DOI 10.1070/RM1988v043n05ABEH001935; English transl., Russian Math. Surveys 43 (1988), no. 5, 221-222. MR.971497(89k:17016) 
[FF2] B. L. Fer̆gin and E. V. Frenkel, Affine Kac-Moody algebras and semi-infinite flag manifolds, Comm. Math. Phys. 128 (1990), no. 1, 161-189. MR1042449 (92f:17026)

[Fie] Peter Fiebig, The combinatorics of category $\mathcal{O}$ over symmetrizable Kac-Moody algebras, Transform. Groups 11 (2006), no. 1, 29-49, DOI 10.1007/s00031-005-1103-8. MR2205072 (2006k:17040)

[FKW] Edward Frenkel, Victor Kac, and Minoru Wakimoto, Characters and fusion rules for $W$ algebras via quantized Drinfel'd-Sokolov reduction, Comm. Math. Phys. 147 (1992), no. 2, 295-328. MR1174415 (93i:17029)

[FM] Igor Frenkel and Fyodor Malikov, Kazhdan-Lusztig tensoring and Harish-Chandra categories. preprint, 1997. arXiv:q-alg/9703010.

[Fre1] Edward Frenkel, Determinant formulas for the free field representations of the Virasoro and Kac-Moody algebras, Phys. Lett. B 286 (1992), no. 1-2, 71-77, DOI 10.1016/03702693(92)90160-6. MR 1175574 (93k:17056)

[Fre2] Edward Frenkel, Wakimoto modules, opers and the center at the critical level, Adv. Math. 195 (2005), no. 2, 297-404, DOI 10.1016/j.aim.2004.08.002. MR2146349(2006d:17018)

[GL] Howard Garland and James Lepowsky, Lie algebra homology and the Macdonald-Kac formulas, Invent. Math. 34 (1976), no. 1, 37-76. MR0414645 (54 \#2744)

[HT] Shinobu Hosono and Akihiro Tsuchiya, Lie algebra cohomology and $N=2$ SCFT based on the GKO construction, Comm. Math. Phys. 136 (1991), no. 3, 451-486. MR.1099691 (92i:17042)

[Kos] Bertram Kostant, Lie algebra cohomology and the generalized Borel-Weil theorem, Ann. of Math. (2) 74 (1961), 329-387. MR0142696 (26 \#265)

[KRW] Victor Kac, Shi-Shyr Roan, and Minoru Wakimoto, Quantum reduction for affine superalgebras, Comm. Math. Phys. 241 (2003), no. 2-3, 307-342. MR2013802(2004h:17024)

[KT] Masaki Kashiwara and Toshiyuki Tanisaki, Kazhdan-Lusztig conjecture for symmetrizable Kac-Moody Lie algebras. III. Positive rational case, Asian J. Math. 2 (1998), no. 4, 779832. Mikio Sato: a great Japanese mathematician of the twentieth century. MR:1734129 (2001f:17050)

[KW1] Victor G. Kac and Minoru Wakimoto, Modular invariant representations of infinitedimensional Lie algebras and superalgebras, Proc. Nat. Acad. Sci. U.S.A. 85 (1988), no. 14, 4956-4960, DOI 10.1073/pnas.85.14.4956. MR.949675 (89j:17019)

[KW2] V. G. Kac and M. Wakimoto, Classification of modular invariant representations of affine algebras, Infinite-dimensional Lie algebras and groups (Luminy-Marseille, 1988), Adv. Ser. Math. Phys., vol. 7, World Sci. Publ., Teaneck, NJ, 1989, pp. 138-177. MR1026952 (91a:17032)

[KW3] Victor G. Kac and Minoru Wakimoto, On rationality of W-algebras, Transform. Groups 13 (2008), no. 3-4, 671-713, DOI 10.1007/s00031-008-9028-7. MR 2452611(2009i:17042)

[Lus] George Lusztig, Hecke algebras and Jantzen's generic decomposition patterns, Adv. in Math. 37 (1980), no. 2, 121-164, DOI 10.1016/0001-8708(80)90031-6. MR591724 (82b:20059)

[MF] F. G. Malikov and I. B. Frenkel', Annihilating ideals and tilting functors (Russian, with Russian summary), Funktsional. Anal. i Prilozhen. 33 (1999), no. 2, 31-42, 95, DOI 10.1007/BF02465191; English transl., Funct. Anal. Appl. 33 (1999), no. 2, 106-115. MR $1719326(2002 \mathrm{c}: 17037)$

[Pet] D. Peterson, Quantum cohomology of G/P. Lecture Notes, Cambridge, MA, Spring, Massachusetts Institute of Technology, 1997.

[RCW] Alvany Rocha-Caridi and Nolan R. Wallach, Projective modules over graded Lie algebras. I, Math. Z. 180 (1982), no. 2, 151-177, DOI 10.1007/BF01318901. MR661694(83h:17018)

[Soe1] Wolfgang Soergel, Kazhdan-Lusztig polynomials and a combinatoric[s] for tilting modules, Represent. Theory 1 (1997), 83-114 (electronic), DOI 10.1090/S1088-4165-97-00021-6. MR.1444322(98d:17026)

[Soe2] Wolfgang Soergel, Character formulas for tilting modules over Kac-Moody algebras, Represent. Theory 2 (1998), 432-448 (electronic), DOI 10.1090/S1088-4165-98-00057-0. MR1663141 (2000c:17048)

[TK] Akihiro Tsuchiya and Yukihiro Kanie, Fock space representations of the Virasoro algebra. Intertwining operators, Publ. Res. Inst. Math. Sci. 22 (1986), no. 2, 259-327, DOI 10.2977/prims/1195178069. MR849260(87k:17020) 
[Vor1] Alexander A. Voronov, Semi-infinite homological algebra, Invent. Math. 113 (1993), no. 1, 103-146, DOI 10.1007/BF01244304. MR.1223226 (94f:17021)

[Vor2] Alexander A. Voronov, Semi-infinite induction and Wakimoto modules, Amer. J. Math. 121 (1999), no. 5, 1079-1094. MR.1713301 (2000g:17025)

[Wak] Minoru Wakimoto, Fock representations of the affine Lie algebra $A_{1}^{(1)}$, Comm. Math. Phys. 104 (1986), no. 4, 605-609. MR841673(87m:17011)

Research Institute for Mathematical Sciences, Kyoto University, Kyoto 606-8502 JAPAN

E-mail address: arakawa@kurims.kyoto-u.ac.jp 